\title{
The Center of the 3-Dimensional and 4-Dimensional Sklyanin Algebras
}

\author{
S. P. SMITH \\ Department of Mathematics, University of Washington, Seattle, WA 98195, U.S.A. \\ e-mail: smith@math.washington.edu \\ and \\ J. TATE \\ Department of Mathematics, University of Texas, Austin, TX 78712, U.S.A. \\ e-mail: tate@math.utexas.edu
}

(Received: April, 1993)

\begin{abstract}
Let $A=A(E, \tau)$ denote either the 3-dimensional or 4-dimensional Sklyanin algebra associated to an elliptic curve $E$ and a point $\tau \in E$. Assume that the base field is algebraically closed, and that its characteristic does not divide the dimension of $A$. It is known that $A$ is a finite module over its center if and only if $\tau$ is of finite order. Generators and defining relations for the center $Z(A)$ are given. If

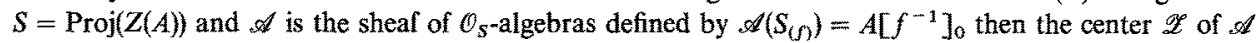
is described. For example, for the 3-dimensional Sklyanin algebra we obtain a new proof of M. Artin's result that Spec $\mathscr{Z} \cong \mathbb{p}^{2}$. However, for the 4-dimensional Sklyanin algebra there is not such a simple result: although Spec $\mathscr{Z}$ is rational and normal, it is singular. We describe its singular locus, which is also the non-Azumaya locus of $\mathscr{A}$.
\end{abstract}

Key words. Sklyanin algebras, center.

\section{Introduction}

Let $k$ be an algebraically closed field, $E$ an elliptic curve over $k$, and $\tau \in E$. For each integer $d \geqslant 3$, the $d$-dimensional Sklyanin algebra $A_{d}(E, \tau)$ is a graded $k$-algebra determined up to isomorphism by that data: it is a Noetherian domain, generated by $d$ elements of degree 1 , and has the same Hilbert series as the polynomial ring in $d$ indeterminates [16]. A precise definition in the cases $d=3$ and $d=4$ treated in this paper is given below. Those cases have been studied in some detail. In particular, it is shown in [5] for $d=3$, and in [14] for $d=4$, that $A$ is a finite module over its center $Z(A)$ if and only if $\tau$ is of finite order. This paper gives an explicit construction and description of $Z(A)$ when $\tau$ is of finite order.

After [1], it is clear that $Z(A)$ is not the only, or even the best thing to study. One should also study the center of the sheaf of algebras $\mathscr{A}$, defined as follows. First let $S:=\operatorname{Proj}(Z(A))$, and for $0 \neq f \in Z(A)_{i}$ write $S_{(f)}=\operatorname{Spec}\left(Z(A)\left[f^{-1}\right]_{0}\right)$ as usual 
[8, Chapter II, §2]. Then $\mathscr{A}$ is the sheaf of $\mathscr{O}_{S}$-algebras defined by

$$
\mathscr{A}\left(S_{(f)}\right):=A\left[f^{-1}\right]_{0} \text {. }
$$

The center $\mathscr{Z}$ of $\mathscr{A}$ is defined by $\mathscr{Z}(U)=Z(\mathscr{A}(U))$ for an open $U \subset S$; in particular $\mathscr{Z}\left(S_{(f)}\right)=Z\left(A\left[f^{-1}\right]_{0}\right)$. It is possible that $\mathscr{Z}$ may be strictly larger than $\mathscr{O}_{s}$. Consider the scheme Spec $\mathscr{Z}$ as defined in [8, Chapter II, Ex 5.17]. When $d=3$ it is proved in [1] that Spec $\mathscr{Z} \cong \mathbb{P}^{2}$. In this paper we give an alternative proof of this result, and also describe Spec $\mathscr{Z}$ when $d=4$.

DEFINITION. Fix an invertible $\mathscr{O}_{E}$-module $\mathscr{L}$ of degree $d$ equal to either 3 or 4 . Set $U=H^{0}(E, \mathscr{L})$ and identify $U \otimes U$ with $H^{0}(E \times E, \mathscr{L} \otimes \mathscr{L})$. Define the shifted diagonal

$$
\Delta_{\tau}:=\{(x, x+\tau) \mid x \in E\}
$$

If $d=3$ set $\Gamma=\Delta_{\tau}$. If $d=4$ set

$$
\Gamma=\Delta_{\tau} \cup\left\{\left(e_{i}, e_{i}\right) \mid 0 \leqslant i \leqslant 3\right\}
$$

where the $e_{i}$ are defined as follows. The image of $E$ in $\mathbb{P}\left(H^{0}(E, \mathscr{L})^{*}\right) \cong \mathbb{P}^{3}$ is contained in a pencil of quadrics, four of which are singular of rank 3: label their four singular points $e_{0}, \ldots, e_{3}$.

The d-dimensional Sklyanin algebra associated to $(E, \tau)$ is defined to be the quotient of the tensor algebra

$$
A_{d}(E, \tau):=T(U) /\left(R_{A}\right)
$$

where

$$
R_{A}:=\left\{f \in U \otimes U|f|_{\Gamma}=0\right\} .
$$

Our definition of these Sklyanin algebras differs from the definition of the higher Sklyanin algebras $Q_{d}(E, \tau)$ given by Odesskii and Feigin in [12]. The relation between the definitions is that $A_{3}(E, \tau)=Q_{3}(E, \tau)$ and $A_{4}(E, 2 \tau)=Q_{4}(E, \tau)$.

Since $E$ and $\tau$ will be fixed throughout the paper, we will just write $A$ for $A_{d}(E, \tau)$. The dependence of the algebra on $\mathscr{L}$ is illusory, since any two line bundles of degree $d$ are pullbacks of one another along suitable translations.

The study of $A$ is facilitated by its having a quotient ring which may be studied more directly. Let $\sigma \in \operatorname{Aut}(E)$ be defined by $p^{\sigma}=p+\tau$, and let $B=B(E, \sigma, \mathscr{L})$ be the twisted homogeneous coordinate ring of $E$ as defined at the beginning of Section 2. By [4, Section 7] $B$ is generated in degree 1 , so there is a surjective map $T(U) \rightarrow B$. If $f \in U \otimes U$ is a relation for $A$, then $f\left(p, p^{\sigma}\right)=0$ so the image of $f$ in $B$ is zero. Hence there is a surjective algebra homomorphism $A \rightarrow$ $B(E, \sigma, \mathscr{L})$.

If $d=3$ then $B(E, \sigma, \mathscr{L}) \cong A /(g)$ where $g$ is a homogeneous central regular element of degree 3 in $A$ [4, Theorem 6.8]. If $d=4$ then $B(E, \sigma, \mathscr{L}) \cong A /\left(\Omega_{1}, \Omega_{2}\right)$ where $\Omega_{1}$ and $\Omega_{2}$ are homogeneous central regular elements of degree 2 which 
form a regular sequence on $A$ [13, Corollary 3.9]. Hence, for both $d=3$ and $d=4$, we may write

$$
A_{d}(E, \tau)=k\left[x_{1}, \ldots, x_{d}\right] \text { and } B(E, \sigma, \mathscr{L})=A /\left(g_{1}, \ldots, g_{m}\right),
$$

where $g_{1}, \ldots, g_{m}, m=d-2$, is a regular sequence of homogeneous central elements, all of degree $a=6-d$.

From now on assume that $\tau$ (equivalently $\sigma$ ) is of finite order $n$. The strategy of the paper is to determine generators for the center of $B$ and then lift these to central elements in $A$, which together with $g_{1}, \ldots, g_{m}$ generate the center of $A$. Unfortunately, matters are complicated by the fact that we also wish to study $\mathscr{Z}$, because Spec $\mathscr{Z}=\operatorname{Proj}\left(Z\left(A^{(b)}\right)\right)$ where $b=\operatorname{gcd}(a, n)$. Consequently, Sections 2 and 3 study $Z\left(A^{(r)}\right)$ for an arbitrary $r$ which divides $b$. Actually Section 2 treats a more general situation. It deals with an arbitrary triple $(X, \sigma, \mathscr{L})$ with the property that $\sigma$ is of finite order $n=r s$, the group generated by $\sigma$ acts freely on $X$ and $\left(\sigma^{\mathrm{s}}\right)^{*} \mathscr{L} \cong \mathscr{L}$. The choice of a map giving this isomorphism induces an automorphism $\rho$ of $B(X, \sigma, \mathscr{L})$ of order $r$ and an automorphism $\tau$ of $B(X, \sigma, \mathscr{L})^{(\mathrm{s})}$ of order $n$. In the Sklyanin situation $\rho$ may be lifted from $B(E, \sigma, \mathscr{L})$ to $A$. The lifted automorphism is still denoted by $\rho$. The restriction to $E$ of the transpose of $\rho$ on $\mathbb{P}\left(A_{1}^{*}\right)$ coincides with $\sigma^{s}$. In a similar way the action of $\tau$ linearizes $\sigma$. The center of a twisted homogeneous coordinate ring $B(X, \sigma, \mathscr{L})$ has a precise description as a homogeneous coordinate ring of the quotient variety $X /\langle\sigma\rangle$. The $r$ th Veronese subalgebra $B(X, \sigma, \mathscr{L})^{(r)}$ is still a twisted homogeneous coordinate ring, so there is a similar description of its center.

Applying this to the Sklyanin situation one obtains a basis $x_{1}, \ldots, x_{d}$ for $B(E, \sigma, \mathscr{L})_{1}$ consisting of $\rho$-eigenvectors such that $Z\left(B^{(r)}\right)=k\left[x_{1}^{s}, \ldots, x_{d}^{s}\right]^{(n)}$. Furthermore, $k\left[x_{1}^{s}, \ldots, x_{d}^{s}\right]$ is isomorphic to a twisted homogeneous coordinate ring of the isogenous curve $E^{\prime}:=E /\left\langle\sigma^{r}\right\rangle$. Of course, one does not expect the elements $x_{i}^{s}$ to be central in $B(E, \sigma, \mathscr{L})$, but they are normalizing elements. Moreover, each $x_{i}^{s}$ may be lifted to an element $u_{i} \in A_{s}$ which is normalizing in $A$; for example, if $x_{i}$ is $\rho$-invariant then $u_{i}$ is $\rho$-normalizing. To show that the elements $x_{i}^{s}$ may be lifted requires a careful analysis of $\rho$-derivations of $B(E, \sigma, \mathscr{L})$. Sections 2 and 3 culminate in Theorem 3.7 which proves that

$$
Z\left(A^{(b)}\right)=k\left[u_{1}, \ldots, u_{d}\right]^{(n)}\left[g_{1}, \ldots, g_{m}\right] \quad \text { and } \quad Z(A)=k\left[z_{1}, \ldots, z_{d}\right]\left[g_{1}, \ldots, g_{m}\right]
$$

where the $u_{i}$ and $z_{i}$ are homogeneous of degrees $s:=n / b$ and $n$ respectively.

Section 4 determines the defining relations for $Z(A)$ and for $Z\left(A^{(b)}\right)$. The first step is to obtain relations for $k\left[u_{1}, \ldots, u_{d}\right]$ by proving it is a twist of a polynomial ring. This polynomial ring has a natural description in terms of the original data: it is $B\left(\mathbb{P}^{\prime}, 1, \mathcal{O}_{\mathbb{P}^{\prime}}(1)\right)$ where $\mathbb{P}^{\prime}=\mathbb{P}\left(H^{\circ}\left(E^{\prime}, \mathscr{L}^{\prime}\right)^{*}\right)$ and $\mathscr{L}^{\prime}$ is the descent of $\mathscr{L}_{s}:=\mathscr{L} \otimes \mathscr{L}^{\sigma} \otimes \ldots \otimes \mathscr{L}^{\sigma^{s-1}}$ to $E^{\prime}$. It is straightforward to obtain a surjective map from the twisted polynomial ring to $k\left[u_{1}, \ldots, u_{d}\right]$ because the latter has relations of the form $u_{i} u_{j}=\alpha_{i j} u_{j} u_{i}$ for suitable $\alpha_{i j} \in k$. However, to show this map is an 
isomorphism one must show that $k\left[u_{1}, \ldots, u_{d}\right]$ has plenty of line modules. These are obtained as submodules of the line modules for $A$. Further analysis of the action of $k\left[u_{1}, \ldots, u_{d}\right]$ on these line modules leads to defining relations for $k\left[u_{1}, \ldots, u_{d}\right]\left[g_{1}, \ldots, g_{m}\right]$ and to defining relations for $Z(A)$.

Suppose that $d=3$. Then $k\left[u_{1}, u_{2}, u_{3}, g\right]$ has defining relations

$$
u_{2} u_{1}=\zeta^{s} u_{1} u_{2}, u_{3} u_{2}=\zeta^{s} u_{2} u_{3}, u_{1} u_{3}=\zeta^{s} u_{3} u_{1}, g^{s}+f_{3}\left(u_{1}, u_{2}, u_{3}\right)=0,
$$

where $\zeta$ is a cube root of 1 and $f_{3}$ is a homogeneous cubic defining $E^{\prime} \subset \mathbb{P}^{\prime}$. The center $Z(A)=k\left[z_{1}, z_{2}, z_{3}, g\right]$ has defining relations

$$
z_{2} z_{1}=z_{1} z_{2}, z_{3} z_{2}=z_{2} z_{3}, z_{1} z_{3}=z_{3} z_{1}, \quad F\left(z_{1}, z_{2}, z_{3}, g\right)=0,
$$

where $F$ is of the form

$$
F\left(z_{1}, z_{2}, z_{3}, g\right):=g^{s}+f_{3}\left(u_{1}, u_{2}, u_{3}\right), \quad \text { if }(3, n)=1
$$

and

$$
\begin{aligned}
F\left(z_{1}, z_{2}, z_{3}, g\right):= & g^{n}+f_{1}\left(z_{1}, z_{2}, z_{3}\right) g^{2 n / 3}+f_{2}\left(z_{1}, z_{2}, z_{3}\right) g^{n / 3}+ \\
& +f_{3}\left(z_{1}, z_{2}, z_{3}\right), \text { if } 3 \mid n,
\end{aligned}
$$

where $f_{3}$ is a cubic defining the image of $E^{\prime \prime}:=E /\langle\sigma\rangle$ in a suitable $\mathbb{P}^{2}$ and $f_{2}$ and $f_{1}$ are nonzero quadratic and linear forms, respectively. We also remark that we can, and do, arrange that $z_{i}=u_{i}^{b}$. There are analogous relations for $d=4$.

Section 5 describes the structure of $\operatorname{Spec} \mathscr{Z}$. When $d=3$ Theorem 5.3 proves that $\operatorname{Spec} \mathscr{Z} \cong \mathbb{P}^{\prime} \cong \mathbb{P}^{2}$. This has already been proved when $(3, n)=1$ in [1] and [3]. When $d=4$ we are unable to give such a concise description of Spec $\mathscr{Z}$. However, we prove that $\operatorname{Spec} \mathscr{Z}$ is a normal singular variety, that it is rational, and that there is a flat morphism Spec $\mathscr{Z} \rightarrow \mathbb{P}^{\prime}$ of degree $s$ induced by the inclusion $k\left[u_{1}, \ldots, u_{4}\right]^{(n)} \subset Z\left(A^{(b)}\right)$ (when $d=3$ the analogous morphism is an isomorphism). We also show that $\mathscr{A}$, which is a sheaf of maximal orders on Spec $\mathscr{Z}$, is nonAzumaya precisely at the singular points, and we give a precise description of this locus.

We will work over a fixed algebraically closed base field $k$. The following constructions and notations will be used in the paper.

Let $R$ be a $\mathbb{Z}$-graded $k$-algebra, and let $M$ be a graded left $R$-module.

The $m$ th-Veronese subalgebra of $R$ is defined to be $R^{(m)}:=\oplus_{i \in \mathbb{Z}} R_{i m}$. The $m$ thVeronese submodule of $M$ is $M^{(m)}:=\bigoplus_{i \in \mathbb{Z}} M_{i m}$.

Let $\theta \in \operatorname{Aut}(R)$ be a $k$-linear algebra automorphism which respects the grading. Then we may define a new algebra structure, $\left(R^{\theta}, *\right)$, on $R$ by declaring that the product of $x \in R_{i}$ and $y \in R_{j}$ is $x * y=x y^{\theta^{i}}$. We call $\left(R^{\theta}, *\right)$ the twist of $R$ with respect to $\theta$.

The center of $R$ will be denoted by $Z(R)$.

Let $\theta \in \operatorname{Aut}(R)$. An element $u \in R$ is $\theta$-normalizing if $u v=v^{\theta} u$ for all $v \in R$. The set of all $\theta$-normalizing elements is a $Z(R)$-submodule of $R$. In a domain a $\theta$ normalizing element is $\theta$-invariant. 
Let $\theta \in \operatorname{Aut}(R)$. A $k$-linear map $\delta: R \rightarrow R$ is a $\theta$-derivation if $\delta(x y)=\delta(x) y+x^{\theta} \delta(y)$ for all $x, y \in R$. If $u \in R$, the inner $\theta$-derivation by $u$ is the map $x \rightarrow u x-x^{\theta} u$.

If $c \in k^{\times}$define $\lambda_{c} \in \operatorname{Aut}(R)$ by $\lambda_{c}(x)=c^{n} x$ for $x \in R_{n}$.

If $d \in \mathbb{Z}$ then $M[d]$ is the graded $R$-module which is equal to $M$ as an ungraded $R$-module but has the grading $M[d]_{i}:=M_{d+i}$.

We say that $M$ is bounded below if $M_{i}=0$ for $i \ll 0$. We say that $M$ is locally finite if $\operatorname{dim}\left(M_{i}\right)<\infty$ for all $i$. If $M$ is locally finite, the Hilbert series of $M$ is the formal power series $H_{M}(t)=\Sigma_{i}\left(\operatorname{dim} M_{i}\right) t^{i}$.

A d-linear $R$-module is a cyclic left $R$-module $M$ having Hilbert series $H_{M}(t)=(1-t)^{-d}$. We call $M$ a point module if $d=1$ and a line module if $d=2$.

Let $X$ be a scheme over $k$, and let $v$ be a $k$-automorphism of $X$.

If $p \in X$ we will usually write $p^{v}$ for $v(p)$. We extend this to Weil divisors in the obvious fashion. For example, if $D=\Sigma n_{p} .(p)$ is a divisor on a curve then $D^{v}:=\Sigma n_{p} \cdot\left(p^{v}\right)$.

Let $f: \mathscr{F} \rightarrow \mathscr{G}$ be a homomorphism of $\mathcal{O}_{X}$-modules. We will write $\mathscr{F}^{v}$ for the inverse image $v^{*} \mathscr{F}$. We note that $\mathscr{F}^{v}=\left(v^{-1}\right)_{*} \mathscr{F}$, and if $D$ is a Weil divisor $\mathcal{O}_{X}(D)^{v}=\mathcal{O}_{X}\left(D^{v-1}\right)$. We will write $f^{v}=v^{*}(f): \mathscr{F}^{v} \rightarrow \mathscr{G}^{v}$ for the homomorphism corresponding to $f$. There is a $k$-linear isomorphism

$$
H^{\circ}(X, \mathscr{F}) \rightarrow H^{0}\left(X, \mathscr{F}^{v}\right)=H^{\circ}\left(X, \mathcal{O}_{X} \otimes_{v-1} \mathscr{Q}_{X} v^{-1} \mathscr{F}\right)
$$

given by $s \rightarrow s^{v}:=1 \otimes s$. Notice that $s\left(p^{v}\right)=0$ if and only if $s^{v}(p)=0$. These notations are compatible: the natural isomorphism $\operatorname{Hom}_{X}\left(\mathcal{O}_{X}, \mathscr{F}\right) \stackrel{\sim}{\rightarrow} H^{0}(X, \mathscr{F})$ given by $f \rightarrow f(1)$ satisfies $f^{v} \rightarrow f(1)^{v}$.

If $X$ is an irreducible variety we write $K=k(X)$ for its function field and $\mathscr{K}_{X}$ for its sheaf of total quotient rings. There is an induced automorphism $f \rightarrow f^{v}$ of $K$ defined by $f^{v}(p)=f\left(p^{v}\right)$. This is compatible with all the previous notation: if $\mathscr{F}$ is a subsheaf of $\mathscr{K}_{X}$ and $f \in H^{0}(X, \mathscr{F})$ then the two possible meanings of $f^{v} \in H^{0}\left(X, \mathscr{F}^{v}\right)$ are the same. We also note that, if $f \in K$ then $\left(f^{v}\right)=(f)^{v^{-1}}$.

\section{Twisted Homogeneous Coordinate Rings}

We begin by recalling some results and notation from [5], [6] and [11].

The category of triples over $k$ has as its objects triples $(X, \sigma \mathscr{L})$ consisting of a $k$-scheme $X$, a $k$-automorphism $\sigma$ of $X$ and an invertible $\mathcal{O}_{X}$-module $\mathscr{L}$. A morphism in this category is a pair

$$
(f, u):(X, \sigma, \mathscr{L}) \rightarrow\left(X^{\prime}, \sigma^{\prime}, \mathscr{L}^{\prime}\right)
$$

consisting of a morphism $f: X \rightarrow X^{\prime}$ such that $\sigma^{\prime} f=f \sigma$, and an $\mathscr{O}_{X}$-module homomorphism $u: f^{*} \mathscr{L}^{\prime} \rightarrow \mathscr{L}$. If $(g, v):\left(X^{\prime}, \sigma^{\prime}, \mathscr{L}^{\prime}\right) \rightarrow\left(X^{\prime \prime}, \sigma^{\prime \prime}, \mathscr{L}^{\prime \prime}\right)$ is also a morphism then $(g, v) \circ(f, u)=\left(g f, u^{\circ} f^{*} v\right)$. 
Define

$$
\mathscr{L}_{n}= \begin{cases}\mathscr{L} \otimes \mathscr{L}^{\sigma} \otimes \ldots \otimes \mathscr{L}^{\sigma^{n-1}}, & \text { if } n>0, \\ \mathcal{O}_{X}, & \text { if } n=0, \\ \mathscr{L}^{-\sigma^{n}} \otimes \ldots \otimes \mathscr{L}^{-\sigma^{-2}} \otimes \mathscr{L}^{-\sigma^{-1}}, & \text { if } n<0 .\end{cases}
$$

There is a contravariant functor $B$ from the category of triples to the category of graded $k$-algebras defined as follows:

$$
B(X, \sigma, \mathscr{L})=\bigoplus_{n \in \mathbb{Z}} B_{n}
$$

where $B_{n}=H^{0}\left(X, \mathscr{L}_{n}\right)$, and the multiplication $B_{n} \otimes B_{m} \rightarrow B_{n+m}$ is given by the composition

$$
\begin{aligned}
& H^{0}\left(X, \mathscr{L}_{n}\right) \otimes H^{0}\left(X, \mathscr{L}_{m}\right) \rightarrow H^{0}\left(X, \mathscr{L}_{n}\right) \otimes H^{0}\left(X,\left(\mathscr{L}_{m}\right)^{\sigma^{n}}\right) \rightarrow H^{0}\left(X, \mathscr{L}_{n} \otimes\left(\mathscr{L}_{m}\right)^{\sigma^{n}}\right) \\
& \quad=H^{0}\left(X, \mathscr{L}_{n+m}\right)
\end{aligned}
$$

where the first of these maps is $a \otimes b \rightarrow a \otimes b^{\sigma^{n}}$.

We say that $\mathscr{L}$ is $\sigma$-ample, if for every coherent $\mathcal{O}_{X}$-module $\mathscr{F}$ and every $q>0$, $H^{q}\left(X, \mathscr{L}_{i} \otimes \mathscr{F}\right)=0$ for $i \gg 0$. We call $B$ a twisted homogeneous coordinate ring of $X$ if $\mathscr{L}$ is both ample and $\sigma$-ample.

Remarks. (1) If $(f, u)$ is a morphism of triples as above, then the induced algebra homomorphism $\varphi: B\left(X^{\prime}, \sigma^{\prime}, \mathscr{L}^{\prime}\right) \rightarrow B(X, \sigma, \mathscr{L})$ is given by $\varphi(b)=$ $\left(u \otimes u^{\sigma} \otimes \ldots \otimes u^{\sigma^{n-1}}\right)\left(f^{*}(b)\right)$ for $b \in B\left(X^{\prime}, \sigma^{\prime}, \mathscr{L}^{\prime}\right)_{n}$ if $n>0$.

(2) If $f \in B_{1} \otimes B_{1}=H^{0}(X \times X, \mathscr{L} \otimes \mathscr{L})$ is in the kernel of the multiplication map to $B_{2}$ then $f\left(p, p^{\sigma}\right)=0$ for all $p \in X$.

Under quite mild restrictions a natural localization of $B(X, \sigma, \mathscr{L})$ is an Ore extension of the function field $K=k(X)$ of $X$. Let $X$ be an irreducible and reduced projective $k$-scheme, let $\xi=\operatorname{Spec}(K)$ denote the generic point of $X$, let $\sigma$ denote the restriction of $\sigma$ to $\xi$, and let $\mathscr{L}_{\xi}$ denote the inverse image of $\mathscr{L}$ on $\xi$. Let $K\left[t, t^{-1} ; \sigma\right]$ be the Ore extension having $K$-basis $\left\{t^{j} \mid j \in \mathbb{Z}\right\}$ as a left $K$-module, and multiplication defined by $t f=f^{\sigma} t$ for $f \in K$. The obvious morphism of triples $\left(\xi, \sigma, \mathscr{L}_{\xi}\right) \rightarrow(X, \sigma, \mathscr{L})$ induces an injective algebra homomorphism $B(X, \sigma, \mathscr{L}) \rightarrow B\left(\xi, \sigma, \mathscr{L}_{\xi}\right)$, and we obtain the following result.

PROPOSITION 2.1. Let $(X, \sigma, \mathscr{L})$ be a triple and suppose that $X$ is an irreducible and reduced projective $k$-scheme. There is an isomorphism $B\left(\xi, \sigma, \mathscr{L}_{\xi}\right) \cong K\left[t, t^{-1} ; \sigma\right]$, and if $\mathscr{L}$ is $\sigma$-ample, then

(a) $B\left(\xi, \sigma, \mathscr{L}_{\xi}\right)$ is a localization of $B(X, \sigma, \mathscr{L})$;

(b) if $\sigma$ is of finite order, then $B\left(\xi, \sigma, \mathscr{L}_{\xi}\right)$ is a central localization of $B(X, \sigma, \mathscr{L})$.

Proof. Since $\xi$ is an integral scheme, $B\left(\xi, \sigma, \mathscr{L}_{\xi}\right)$ is a domain. In particular, the multiplication map in $B\left(\xi, \sigma, \mathscr{L}_{\xi}\right)$ gives each $B\left(\xi, \sigma, \mathscr{L}_{\xi}\right)_{i}$ the structure of a 1 dimensional vector space over $B\left(\xi, \sigma, \mathscr{L}_{\xi}\right)_{0}=K$. If $0 \neq x \in B\left(\xi, \sigma, \mathscr{L}_{\xi}\right)_{1}$ then 
$B\left(\xi, \sigma, \mathscr{L}_{\xi}\right)_{i}=K x^{i}$. Hence, there is an algebra isomorphism $\varphi_{x}: B\left(\xi, \sigma, \mathscr{L}_{\xi}\right) \rightarrow$ $K\left[t, t^{-1} ; \sigma\right]$ given by $\varphi_{x}\left(f x^{i}\right)=f t^{i}$ for all $i \in \mathbb{Z}$ and $f \in K$.

(a) By [6, Proposition 3.2(iii)] we may choose an $n$ such that $\mathscr{L}_{n}$ and $\mathscr{L}_{n+1}$ are very ample. Thus, $B_{n}$ and $B_{n+1}$ are both nonzero, so Fract $B(\mathrm{X}, \sigma, \mathscr{L})$ contains a nonzero element of degree 1. Let $0 \neq u \in H^{0}\left(X, \mathscr{L}_{n}\right)$ and set $D=(u)$. Then $B(X, \sigma, \mathscr{L})\left[u^{-1}\right]$ contains $H^{0}\left(X, \mathcal{O}_{X}(D)\right)$ and, hence, Fract $B(X, \sigma, \mathscr{L})$ contains the subfield of $K$ generated by $H^{0}\left(X, \mathcal{O}_{X}(D)\right)$. But this subfield is $K$ since $\mathcal{O}_{X}(D)$ is very ample. It follows that $B\left(\xi, \sigma, \mathscr{L}_{\xi}\right)$ is the localization of $B(X, \sigma, \mathscr{L})$ at the nonzero homogeneous elements.

(b) If $\sigma$ is of finite order, $n$ say, then $K\left[t, t^{-1} ; \sigma\right]$ has center $K^{\langle\sigma\rangle}\left[t^{n}, t^{-n}\right]$, so $B\left(\xi, \sigma, \mathscr{L}_{\xi}\right)$ is finite over its center. Thus $B(X, \sigma, \mathscr{L})$ is a prime ring satisfying a polynomial identity, whence by Posner's Theorem Fract $B(X, \sigma, \mathscr{L})$ is a central localization of $B(X, \sigma, \mathscr{L})$. Thus, if $r \in B\left(\xi, \sigma, \mathscr{L}_{\xi}\right)$ is homogeneous, then $r z \in B(X, \sigma, \mathscr{L})$ for some nonzero central element $z \in B(X, \sigma, \mathscr{L})$. But each homogeneous component of $z$ is also central, so we may assume that $z$ is homogeneous. Thus $r \in B(X, \sigma, \mathscr{L})\left[z^{-1}\right] \subset$ $B\left(\xi, \sigma, \mathscr{L}_{\xi}\right)$, so $B\left(\xi, \sigma, \mathscr{L}_{\xi}\right)$ is a central localization as claimed.

PROPOSITION 2.2 [5, pp. 374-375]. Suppose that $X$ is projective, reduced and irreducible, and that $\sigma^{s}$ fixes the class of $\mathscr{L}$ in $\operatorname{Pic}(X)$. Let $u: \mathscr{L}^{\sigma^{s}} \stackrel{\sim}{\longrightarrow} \mathscr{L}$ be an isomorphism, and define the isomorphism $v: \mathscr{L}_{s}^{\sigma} \stackrel{\sim}{\longrightarrow} \mathscr{L}_{s}$ by $v\left(x_{1} \otimes \ldots \otimes x_{s}\right)=$ $u\left(x_{s}\right) \otimes x_{1} \otimes \ldots \otimes x_{s-1} . \quad B y \quad$ functoriality $\quad\left(\sigma^{s}, u\right) \in \operatorname{Aut}(X, \sigma, \mathscr{L}), \quad$ and $(\sigma, v) \in$ $\operatorname{Aut}\left(X, \sigma^{s}, \mathscr{L}_{s}\right)$ induce algebra automorphisms

$$
\rho \in \text { Aut } B(X, \sigma, \mathscr{L}) \text { and } \tau \in \text { Aut } B\left(X, \sigma^{s}, \mathscr{L}_{s}\right) \text {. }
$$

Let $x, y \in B_{i}, z \in B_{j}$ and $w \in B_{m s}$ where $i+j=m s$. Then

(a) $x^{\rho^{m}} z y=y^{\rho^{m}} z x$;

(b) $\tau^{i}(z y)=y^{\rho^{m}} z$;

(c) identifying $B\left(X, \sigma^{s}, \mathscr{L}_{s}\right)$ with $B(X, \sigma, \mathscr{L})^{(s)}$, the restriction of $\rho$ to $B(X, \sigma, \mathscr{L})^{(s)}$ equals $\tau^{s}$

(d) $w^{\tau^{i}} y=y^{\rho^{m}} w$.

Proof. Both $\left(\sigma^{s}, u\right)$ and $(\sigma, v)$ extend to automorphisms of the triples $\left(\xi, \sigma, \mathscr{L}_{\xi}\right)$ and $\left(\xi, \sigma^{s},\left(\mathscr{L}_{s}\right)_{\xi}\right)$, so $\rho$ and $\tau$ extend to automorphisms of $B\left(\xi, \sigma, \mathscr{L}_{\xi}\right)$ and $B\left(\xi, \sigma, \mathscr{L}_{\xi}\right)^{(s)}$ respectively. Therefore, it suffices to prove the Proposition for $B\left(\xi, \sigma, \mathscr{L}_{\xi}\right)$ which has the advantage of being generated over its degree 0 component by the elements of degree 1 and -1 . We will only consider the cases $i, j \geqslant 0$.

(a) The case $i=m=1$ is true because

$$
x^{\rho} z y=(u \otimes 1 \otimes 1)\left(x^{\sigma^{s}} \otimes z^{\sigma} \otimes y^{\sigma^{s}}\right)=(u \otimes 1 \otimes 1)\left(y^{\sigma^{s}} \otimes z^{\sigma} \otimes x^{\sigma^{s}}\right)=y^{\rho} z x .
$$

For $i=1$ and a general $m$ we proceed by induction. Suppose that $z=z_{1} z_{2} z_{3}$ with $z_{1} \in B_{s-1}, z_{2} \in B_{1}, z_{3} \in B_{(m-1) s-1}$. Then

$$
x^{\rho^{m}} z y=x^{\rho^{m}} z_{1} z_{2} z_{3} y=z_{2}^{\rho} z_{1} x^{\rho^{m-1}} z_{3} y=z_{2}^{\rho} z_{1} y^{\rho^{m-1}} z_{3} x=y^{\rho^{m}} z_{1} z_{2} z_{3} x=y^{\rho^{m}} z x .
$$


We now prove the general case by induction on $i$. Suppose $x=x_{1} x_{2}$ and $y=y_{1} y_{2}$ with $x_{1}, y_{1} \in B_{i-1}$ and $x_{2}, y_{2} \in B_{1}$. Then

$$
x^{\rho^{m}} z y=\left(x_{1} x_{2}\right)^{\rho^{m}} z y_{1} y_{2}=x_{1}^{\rho^{m}} y_{2}^{\rho^{m}} z y_{1} x_{2}=y_{1}^{\rho^{m}} y_{2}^{\rho^{m}} z x_{1} x_{2}=y^{\rho^{m}} z x \text {. }
$$

(b) The result is true for $i=1$ and $m=1$ because, if $z_{1}, \ldots, z_{s} \in B_{1}$ then

$$
\begin{aligned}
\tau\left(z_{1} \ldots z_{s}\right) & =v\left(\left(z_{1} \ldots z_{s}\right)^{\sigma}\right)=v\left(z_{1}^{\sigma} \otimes z_{2}^{\sigma^{2}} \otimes \ldots \otimes z_{s}^{\sigma^{s}}\right) \\
& =(u \otimes 1 \otimes 1 \otimes \ldots \otimes 1)\left(z_{s}^{\sigma^{s}} \otimes z_{1}^{\sigma} \otimes \ldots \otimes z_{s-1}^{\sigma^{s-1}}\right) \\
& =u\left(z_{s}^{\sigma^{s}}\right) \otimes z_{1}^{\sigma} \otimes \ldots \otimes z_{s-1}^{\sigma^{s-1}} \\
& =z_{s}^{\rho} z_{1} \ldots z_{s-1} .
\end{aligned}
$$

We now prove the case $i=1$ and general $m$ by induction. Suppose that $z=z_{1} \ldots z_{m s-1}$ with each $z_{l} \in B_{1}$. Then

$$
\begin{aligned}
\tau(z y) & =\tau\left(z_{1} \ldots z_{s}\right) \tau\left(z_{s+1} \ldots z_{m s-1} y\right) \\
& =z_{s}^{\rho} z_{1} \ldots z_{s-1} y^{\rho^{m-1}} z_{s+1} \ldots z_{m s-1}=y^{\rho^{m}} z_{1} \ldots z_{m s-1}=y^{\rho} z .
\end{aligned}
$$

For a general $i$ and general $m$ we proceed by induction on $i$. Suppose that $y=y_{1} y_{2}$ with $y_{1} \in B_{1}$ and $y_{2} \in B_{i-1}$. Then

$$
\tau^{i}(z y)=\tau\left(\tau^{i-1}\left(z y_{1} y_{2}\right)\right)=\tau\left(y_{2}^{\rho^{m}} z y_{1}\right)=y_{1}^{\rho^{m}} y_{2}^{\rho^{m}} z=y^{\rho^{m}} z .
$$

(c) This follows by iterating $s$ times the case $i=m=1$ of (b).

(d) Suppose that $w=w_{1} w_{2}$ with $w_{1} \in B_{m s-i}$ and $w_{2} \in B_{i}$. Then, using (a) and (b),

$$
w^{\boldsymbol{x}^{i}} y=\tau^{i}\left(w_{1} w_{2}\right) y=w_{2}^{\rho^{m}} w_{1} y=y^{\rho^{m}} w_{1} w_{2}
$$

as required.

COROLLARY 2.3. Suppose that $X$ is a reduced and irreducible projective $k$-scheme, $\sigma$ is of finite order and $\mathscr{L}$ is $\sigma$-ample. Then $B(X, \sigma, \mathscr{L})$ is finite over its center.

Proof. By the arguments in [6] $B=B(X, \sigma, \mathscr{L})$ is a finite $B^{(m)}$-module for $m \gg 0$. Hence we can choose $n$ such that $\sigma^{n}=1$ and $B$ is a finite $B^{(n)}$-module. Taking $s=n$ and $u=\mathrm{Id}$ in (2.2) gives $\rho=1$ and $\tau \in$ Aut $B^{(n)}$ satisfies $\tau^{n}=1$. By (2.2d) the $\tau$-invariants in $B^{(n)}$ are central in $B$. But $B^{(n)} \cong B\left(X, 1, \mathscr{L}_{n}\right)$ is commutative, so is a finite module over its subring of $\tau$-invariants. Hence, the result.

Remark. The use of Posner's Theorem in the proof of $(2.1 \mathrm{~b})$ can be avoided as follows. To prove (2.1b) we must show that a nonzero homogeneous element $b$ of $B=B(X, \sigma, \mathscr{L})$ divides a central element. However, if $\sigma^{n}=1$ then, as in the proof of (2.3), $\left(b^{n}\right)^{1+\tau+\cdots+\tau^{n-1}}$ is a $\tau$-invariant element of $B^{(n)}$, so central in $B$, and is also a multiple of $b$.

Let $G$ be a finite group acting as $k$-automorphisms of $X$. Suppose further that $G$ acts freely on $X$ (i.e. all isotropy groups are trivial). This ensures that the quotient map $\pi_{G}: X \rightarrow X / G$ is étale. A coherent $G$-sheaf on $X$ is a coherent $\mathcal{O}_{X}$-module $\mathscr{L}$ endowed with $\mathcal{O}_{X}$-module isomorphisms $T_{g}: \mathscr{L} \rightarrow g^{*} \mathscr{L}$ for each $g \in G$, which satisfy 
$T_{g h}=h^{*}\left(T_{g}\right) \circ T_{h}$. We call $\left(\left(\pi_{G}\right)_{*} \mathscr{L}\right)^{G}$ the descent of $\mathscr{L}$ to $X / G$, and we refer to $g \rightarrow T_{g}$ as descent data.

PROPOSITION 2.4 [11, Proposition 2, page 70]. The functors

$$
\mathscr{F} \rightarrow \pi_{G}^{*} \mathscr{F} \quad \text { and } \quad \mathscr{L} \rightarrow\left(\left(\pi_{G}\right)_{*} \mathscr{L}\right)^{G}
$$

are mutually inverse equivalences between the categories of coherent $\mathcal{O}_{X / G}$-modules and coherent $G$-sheaves on $X$. The equivalence sends locally free modules to locally free modules of the same rank.

If $g$ commutes with $\sigma$ then $\left(g, T_{g}^{-1}\right) \in \operatorname{Aut}(X, \sigma, \mathscr{L})$. Hence if every element of $G$ commutes with $\sigma$, then $G$ acts on $B(X, \sigma, \mathscr{L})$ from the right as algebra automorphisms. In this case we will call $(X, \sigma, \mathscr{L})$ a $G$-triple, and will write simply $f^{g}$ instead of $T_{g}^{-1}\left(f^{g}\right)$ for the action of $g$ on a section $f$ of $\mathscr{L}$.

PROPOSITION 2.5. Suppose that $(X, \sigma, \mathscr{L})$ is a $G$-triple, with $G$ acting freely on $X$. Let $\sigma^{\prime}$ be the automorphism of $X / G$ induced by $\sigma$, and let $\mathscr{L}^{\prime}$ be the descent of $\mathscr{L}$ to $X / G$. Let $u: \pi_{G}^{*} \mathscr{L}^{\prime} \rightarrow \mathscr{L}$ be the canonical isomorphism. Then $\left(\pi_{G}, u\right):(X, \sigma, \mathscr{L}) \rightarrow\left(X / G, \sigma^{\prime}, \mathscr{L}^{\prime}\right)$ is a morphism of triples inducing an isomorphism $\varphi: B\left(X / G, \sigma^{\prime}, \mathscr{L}^{\prime}\right) \rightarrow B(X, \sigma, \mathscr{L})^{G}$.

Proof. The key point is that $\left(\mathscr{L}^{\prime}\right)_{m}$ is the descent of $\mathscr{L}_{m}$ to $X / G$ : this is a consequence of the fact that $\pi_{G}^{*}$ distributes across tensor products. Therefore,

$$
B\left(X / G, \sigma^{\prime}, \mathscr{L}^{\prime}\right)_{m}=H^{0}\left(X / G,\left(\left(\pi_{G}\right)_{*} \mathscr{L}_{m}\right)^{G}\right)=B(X, \sigma, \mathscr{L})_{m}^{G}
$$

The result follows.

From now on we suppose that

- $(X, \sigma, \mathscr{L})$ is a triple, such that $\sigma$ is of finite order $n=r s$,

- the group generated by $\sigma$ acts freely on $X$,

- $X$ is projective, reduced and irreducible,

- $\mathscr{L}^{\sigma^{s}} \cong \mathscr{L}$,

- and $\mathscr{L}$ is $\sigma$-ample. By [6, Lemma 4.1] this is equivalent to $\mathscr{L}_{n}$ being ample. In fact, it is equivalent to $\mathscr{L}_{s}$ being ample, since $\mathscr{L}_{s}^{\sigma^{s}} \cong \mathscr{L}_{s}$ implies $\mathscr{L}_{n} \cong\left(\mathscr{L}_{s}\right)^{\otimes r}$.

We will call triples satisfying these hypotheses $(r, s)$-triples. An $(r, s)$-triple is also a $(1, n)$-triple. To an $(r, s)$-triple we attach groups

$$
G=\langle\sigma\rangle, \quad H=\left\langle\sigma^{s}\right\rangle, \quad G^{\prime}=\left\langle\sigma^{r}\right\rangle
$$

of automorphisms of $X$. This gives rise to a commutative diagram of étale morphisms:

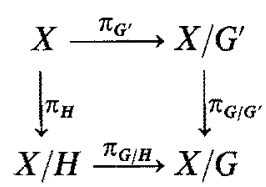

The induced action of $\sigma$ on each of these quotients of $X$ will also be denoted by $\sigma$. 
Since $X$ is projective and irreducible $H^{0}\left(X, \mathcal{O}_{X}\right)=k$. Thus the automorphism group of an invertible $\mathcal{O}_{X}$-module is $k^{\times}$. In particular, the isomorphism $u: \mathscr{L}^{\sigma^{s}} \stackrel{\sim}{\longrightarrow} \mathscr{L}$ is determined up to a scalar multiple. Changing $u$ by a scalar multiple we can, and do from now on, assume that $\left(\sigma^{s}, u\right)^{r}=\mathrm{Id}$, or equivalently $u u^{\sigma^{s}} \ldots u^{\sigma^{(r-1) s}}=$ Id. This determines $u$ up to multiplication by an $r$ th root of 1 . Define $v: \mathscr{L}_{s}^{\sigma} \rightarrow \mathscr{L}_{s}$ as in (2.2). Let $\rho$ and $\tau$ be the automorphisms determined by $\left(\sigma^{s}, u\right)$ and $(\sigma, v)$ as in (2.2). Although $u$, and hence $\tau$, is only determined up to an $r$ th root of unity, $\tau^{r}$ is independent of any such choice. Thus $\tau^{r}$ is a canonical automorphism of $B\left(X, \sigma^{s}, \mathscr{L}_{s}\right)=B(X, \sigma, \mathscr{L})^{(s)}$.

Since $\left(\sigma^{s}, u\right)^{r}=\mathrm{Id}$, the map $H \rightarrow \operatorname{Aut}(X, \sigma, \mathscr{L})$ given by $\sigma^{i s} \rightarrow\left(\sigma^{s}, u\right)^{i}$ is a group homomorphism, whence $\mathscr{L}$ is an $H$-sheaf, with $\left(\sigma^{i s}, T_{\sigma^{i s}}^{-1}\right)=\left(\sigma^{s}, u\right)^{i}$. Furthermore $(\sigma, v)^{n}=\mathrm{Id}$ which ensures that $\mathscr{L}_{s}$ is a $G$-sheaf, and hence a $G^{\prime}$-sheaf. Thus $(X, \sigma, \mathscr{L})$ becomes an $H$-triple, and $\left(X, \sigma^{s}, \mathscr{L}_{s}\right)$ becomes a $G$-triple. The $H$-action on $B(X, \sigma, \mathscr{L})$ is generated by $\rho$, and the $G$-action on $B\left(X, \sigma^{s}, \mathscr{L}_{s}\right)$ is generated by $\tau$. Note that $\rho^{r}=1$ and $\tau^{n}=1$.

PROPOSITION 2.6. Let $(X, \sigma, \mathscr{L})$ be an $(r, s)$-triple, and let $\mathscr{L}^{\prime}$ be the descent of $\mathscr{L}_{s}$ to $X / G^{\prime}$. There is a canonical injective algebra homomorphism

$$
B\left(X / G^{\prime}, \sigma^{s}, \mathscr{L}^{\prime}\right) \rightarrow B(X, \sigma, \mathscr{L})^{(s)}
$$

whose image is the $G^{\prime}=\left\langle\tau^{r}\right\rangle$-invariants of $B(X, \sigma, \mathscr{L})^{(s)}$. This map sends elements of degree $i$ to elements of degree is.

Proof. This map is the composition $B\left(X / G^{\prime}, \sigma^{s}, \mathscr{L}^{\prime}\right) \rightarrow B\left(X, \sigma^{s}, \mathscr{L}_{s}\right) \rightarrow$ $B(X, \sigma, \mathscr{L})^{(s)}$, where the first of these maps arises as in (2.5) using the fact that $\mathscr{L}_{s}$ is a $G^{\prime}$-triple.

Now $G / G^{\prime}$ acts on $B(X, \sigma, \mathscr{L})^{(s) G^{\prime}} \cong B\left(X / G^{\prime}, \sigma^{s}, \mathscr{L}^{\prime}\right)$ with the action generated by the restriction of $\tau$.

THEOREM 2.7. Let $(X, \sigma, \mathscr{L})$ be an $\left(r\right.$, s)-triple. Write $B=B(X, \sigma, \mathscr{L})$ and $B^{\prime}$ for the image in $B$ of $B\left(X / G^{\prime}, \sigma^{s}, \mathscr{L}^{\prime}\right)$ under the canonical map described in (2.6). Fix an integer $m$. Then

$$
\left\{y \in B \mid y x=x^{\rho^{m}} y \text { for all } x \in B^{(r)}\right\}=\bigoplus_{j=m(\bmod r)} B_{j s}^{\prime} .
$$

Proof. To see that the right side is contained in the left side let $y \in B_{j s}^{\prime}$ with $j \equiv m(\bmod r)$. If $x \in B_{i r}$ then by $(2.2 \mathrm{~d}) y^{\tau^{i r}} x=x^{\rho^{j}} y$. But $\rho^{j}=\rho^{m}$ since $\rho^{r}=1$, and elements of $B^{\prime}$ are $\tau^{r}$-invariant by (2.6), so $y x=x^{\rho^{m}} y$ as required.

Conversely suppose that $0 \neq y \in B_{l}$ is in the left side. Let $x \in B_{i r s}$. By (2.2d) $x^{\tau^{l}} y=y^{\rho^{i r}} x=y x=x^{\rho^{m}} y$. Since $B$ is a domain it follows that $\tau^{l}=\rho^{m}$ on $B^{(n)}$. Thus $\tau^{l r}=1$ on $B^{(n)}$. But $\mathscr{L}$ is $\sigma$-ample, so $\tau$ is still of order $n$ on $B^{(n)}$. Thus $l=j$ s for some $j$.

Therefore $\rho^{m}=\tau^{j s}=\rho^{j}$ on $B^{(n)}$ by $(2.2 \mathrm{c})$. By the $\sigma$-ampleness it follows that $r$ divides $j-m$. Finally we show that $y$ is $\tau^{r}$-invariant and, hence, in $B^{\prime}$ by (2.6). Since $\mathscr{L}$ is $\sigma$-ample $B_{i r} \neq 0$ for some $i r \equiv r(\bmod n)$. If $0 \neq x \in B_{i r}$ then by $(2.2 \mathrm{~d})$ 
$y^{\tau^{i r}} x=x^{\rho^{j}} y=x^{\rho^{m}} y=y x$. Thus $y^{\tau^{i r}}=y$ and $y$ is $\tau^{r}$-invariant by the careful choice of $i$.

COROLLARY 2.8. Let $(X, \sigma, \mathscr{L})$ be an $(r, s)$-triple. Let $B\left(X / G^{\prime}, \sigma^{s}, \mathscr{L}^{\prime}\right) \rightarrow$ $B(X, \sigma, \mathscr{L})^{(s)}$ be the canonical map described in (2.6). The restriction of this to the rth Veronese subalgebra gives an isomorphism

$$
B\left(X / G^{\prime}, \sigma^{s}, \mathscr{L}^{\prime}\right)^{(r)} \stackrel{\sim}{\longrightarrow}\left(B(X, \sigma, \mathscr{L})^{(r)}\right) .
$$

This isomorphism sends elements of degree $i$ to elements of degree is.

Proof. If we take $m=0$ in (2.7), we see that the centralizer of $B^{(r)}$ in $B$ is $\left(B^{\prime}\right)^{(n)}$ which is the image of $B\left(X / G^{\prime}, \sigma^{s}, \mathscr{L}^{\prime}\right)^{(r)}$ as required.

Let $\hat{G}=\operatorname{Hom}\left(G, k^{\times}\right)$be the group of $k$-valued characters of $G$. If $\alpha \in \hat{G}$, define

$$
\mathcal{O}_{X / G}(\alpha):=\left\{f \in\left(\pi_{G}\right)_{*} \mathcal{O}_{X} \mid f^{g}=\alpha(g) f \text { for all } g \in G\right\} \text {. }
$$

As remarked in [11], each $\mathcal{O}_{X / G}(\alpha)$ is an invertible $\mathcal{O}_{X / G}$-module, the multiplication in $\left(\pi_{G}\right)_{*} \mathcal{O}_{X}$ induces isomorphisms $\mathcal{O}(\alpha) \otimes_{\mathcal{O}_{X / G}} \mathcal{O}(\beta) \rightarrow \mathcal{O}(\alpha \beta)$, and the map $\alpha \rightarrow \mathcal{O}_{X / G}(\alpha)$ defines an isomorphism

$$
\hat{G} \stackrel{\sim}{\sim} \operatorname{Ker}(\operatorname{Pic}(X / G) \rightarrow \operatorname{Pic}(X)) .
$$

For each $\alpha \in \hat{G}, \mathcal{O}_{X / G}(\alpha)$ is the descent of $\mathcal{O}_{X}$ to $X / G$ with descent data $g \rightarrow(g, \alpha(g))$. If the order of $G$ is prime to the characteristic of $k$, and $G$ is Abelian, then

$$
\left(\pi_{G}\right)_{*} \mathcal{O}_{X}=\bigoplus_{\alpha \in \hat{G}} \mathcal{O}_{X / G}(\alpha)
$$

We now assume that $\operatorname{char}(k)$ does not divide $r$, and apply this to the situation described earlier.

Let $\mathscr{M}$ be the descent of $\mathscr{L}$ to $X / H$, and $\mathscr{L}^{\prime \prime}$ the descent of $\mathscr{L}_{s}$ to $X / G$. We will continue to write $\mathscr{L}^{\prime}$ for the descent of $\mathscr{L}_{s}$ to $X / G^{\prime}$. It is easy to see that $\mathscr{L}^{\prime \prime}$ is the $\left(G / G^{\prime}\right)$-descent of $\mathscr{L}^{\prime}$.

For each $\alpha \in \hat{H}$, define $\mathscr{M}(\alpha)=\mathscr{O}_{X / H}(\alpha) \otimes_{\mathscr{C}_{X / H}} \mathscr{M}$ and for each $\alpha \in\left(G / G^{\prime}\right) \hat{\text {, define }}$ $\mathscr{L}^{\prime \prime}(\alpha)=\mathcal{O}_{X / G}(\alpha) \otimes_{\mathcal{O}_{X / G}} \mathscr{L}^{\prime \prime}$. We obtain two important decompositions:

$$
\begin{aligned}
& H^{0}(X, \mathscr{L})=H^{0}\left(X / H,\left(\pi_{H}\right)_{*} \mathscr{L}\right)=\underset{\alpha \in \cap}{\bigoplus} H^{0}(X / H, \mathscr{M}(\alpha)), \\
& H^{0}\left(X / G^{\prime}, \mathscr{L}^{\prime}\right)=H^{0}\left(X / G,\left(\pi_{G / G^{\prime}}\right)_{*} \mathscr{L}^{\prime}\right)=\bigoplus_{\alpha \in\left(G / G^{\prime}\right)^{\prime}} H^{0}\left(X / G, \mathscr{L}^{\prime \prime}(\alpha)\right) .
\end{aligned}
$$

The first of these is the decomposition of $B(X, \sigma, \mathscr{L})_{1}$ into its $\rho$-eigenspaces, and the second is the decomposition of $B\left(X / G^{\prime}, \sigma^{s}, \mathscr{L}^{\prime}\right)_{1}$ (or equivalently $\left.B(X, \sigma, \mathscr{L})_{s}^{G^{\prime}}\right)$ into its $\tau$-eigenspaces.

For each $\alpha \in \hat{H}, \quad(X / H, \sigma, \mathscr{M}(\alpha))$ is a triple, so we define $\mathscr{H}(\alpha)_{s}=$ $\mathscr{M}(\alpha) \otimes \mathscr{M}(\alpha)^{\sigma} \otimes \ldots \otimes \mathscr{M}(\alpha)^{s-1}$. The isomorphism $G / G^{\prime} \rightarrow H$ given by $g \rightarrow g^{s}$, gives an isomorphism of character groups $\hat{H} \rightarrow\left(G / G^{\prime} \hat{)}\right.$.

PROPOSITION 2.9. Let $\alpha \in \hat{H}=\left(G / G^{\prime}\right)^{\hat{y}}$. Then $\mathscr{L}^{\prime \prime}(\alpha)$ is the descent of $\mathscr{M}(\alpha)_{s}$ to $X / G$. 
Proof. First observe that $\mathscr{M}(\alpha)_{s}$ can be made into a $G / H$-sheaf with descent data $\sigma^{i s} \rightarrow T^{i}$ where $T: \mathscr{M}(\alpha)_{s} \rightarrow \sigma^{*} \mathscr{M}(\alpha)_{s}$ is just the permutation $a_{1} \otimes a_{2} \otimes \ldots \otimes a_{s} \rightarrow$ $a_{2} \otimes \cdots \otimes a_{s} \otimes a_{1}$.

It suffices to show that $\mathcal{O}_{X / G}(\alpha)$ is the descent of $\mathscr{O}_{X / H}(\alpha)_{s}$ to $X / G$. Then $\mathcal{O}_{X / H}(\alpha)=\mathscr{O}_{X / H} f \subset\left(\pi_{H}\right)_{*} \mathscr{K}_{X}$ where $f \in K=H^{0}\left(X, \mathscr{K}_{X}\right)$ satisfies $f^{\sigma^{s}}=\alpha\left(\sigma^{s}\right) f$; the existence of such an $f$ is guaranteed by Kummer theory. Now define $h=f f^{\sigma} \ldots f^{\sigma-1}$. Then $\mathscr{O}_{X / H}(\alpha)_{s}=\mathscr{O}_{X / H} h$ so the $G / H$ descent of $\mathcal{O}_{X / H}(\alpha)_{s}$ to $X / G$ is $\mathscr{O}_{X / G} h$. But $h^{\sigma}=\alpha\left(\sigma^{s}\right) h$ so $\mathscr{O}_{X / G} h=\mathscr{O}_{X / G}(\alpha)$.

PROPOSITION 2.10. Let $E$ be an elliptic curve and $\pi: E \rightarrow E^{\prime}$ a quotient by a finite subgroup. Let $D \in \operatorname{Div}(E)$ and assume that $\operatorname{deg}(D)>0$. Let $K$ and $K^{\prime}$ be the function fields of $E$ and $E^{\prime}$. The restriction of the norm mapping $N: K \rightarrow K^{\prime}$ gives a surjective map from $H^{\circ}\left(E, \mathcal{O}_{E}(D)\right)$ to $H^{\circ}\left(E^{\prime}, \mathcal{O}_{E^{\prime}}(\pi(D))\right)$.

Proof. Set $D^{\prime}=\pi(D)$. Let $0 \neq f^{\prime} \in H^{0}\left(E^{\prime}, \mathcal{O}_{E^{\prime}}\left(D^{\prime}\right)\right)$. Suppose that its divisor is

$$
\operatorname{div}\left(f^{\prime}\right)=\sum_{i=1}^{d}\left(p_{i}^{\prime}\right)-D^{\prime}
$$

Choose preimages $p_{1}, \ldots, p_{d-1} \in E$ of $p_{1}^{\prime}, \ldots, p_{d-1}^{\prime}$. Because $E$ is an elliptic curve, there is a unique point $q \in E$ such that $D$ is linearly equivalent to $\left(p_{1}\right)+\cdots+\left(p_{d-1}\right)+(q)$. Let $f \in K$ be such that $\operatorname{div}(f)=\left(p_{1}\right)+\cdots+\left(p_{d-1}\right)+$ $(q)-D$. Then $f \in H^{0}\left(E, \mathcal{O}_{E}(D)\right)$ and

$$
\operatorname{div}(N f)=\left(p_{1}^{\prime}\right)+\cdots+\left(p_{d-1}^{\prime}\right)+\left(q^{\prime}\right)-D^{\prime}
$$

where $q^{\prime}$ is the image of $q$ in $E^{\prime}$. It follows that $q^{\prime}$ is linearly equivalent to $p_{d}^{\prime}$. Since $E^{\prime}$ is not a rational curve this forces $q^{\prime}=p_{d}^{\prime}$. Hence, $f^{\prime}=c \cdot N(f)=N\left(c^{1 / n} f\right)$ for some $c \in k$.

COROLLARY 2.11. Let $X$ be an elliptic curve and $\sigma$ the translation by a point of finite order. Then the map $B_{1} \rightarrow B_{s}$ given by $y \rightarrow y^{s}$ gives, on restriction, a surjective mapping $H^{0}(X / H, \mathscr{M}(\alpha))$ to $H^{0}\left(X / G, \mathscr{L}^{\prime \prime}(\alpha)\right)$ for each $\alpha \in \hat{H}$.

Proof. Both $E:=X / H$ and $E^{\prime}:=X / G$ are elliptic curves and $\pi_{G / H}: E \rightarrow E^{\prime}$ is as in the previous result. Indeed $\pi_{G / H}$ is a cyclic étale cover with Galois group $G / H$.

Since $\mathscr{L}^{\prime \prime}(\alpha)$ is the descent of $\mathscr{M}(\alpha)$, one of these has only the zero section if and only if the other does. Suppose that $0 \neq x \in H^{0}(X / H, \mathscr{M}(\alpha))$ and set $D=\operatorname{div}(x) \in \operatorname{Div}(E)$. Thus $\mathscr{M}(\alpha)=\mathcal{O}_{E}(D) x$ and $x^{\rho}=\alpha(\rho) x$.

Put $y=x^{s-1}$ in (2.2b): thus $\left(x^{s}\right)^{z}=x^{\rho} x^{s-1}=\alpha\left(\tau^{s}\right) x^{s}$. Hence, $x^{s}$ is $G^{\prime}$-invariant and $x^{s} \in H^{0}\left(X / G, \mathscr{L}^{\prime \prime}(\alpha)\right)$. Since $x^{s}=x \otimes x^{\sigma} \otimes \ldots \otimes x^{\sigma^{s-1}}$ the divisor of $x^{s}$ as a section over $X / G$ is $\pi_{G / H}(D)$. Thus $\mathscr{L}^{\prime \prime}(\alpha)=\mathcal{O}_{E^{\prime}}\left(\pi_{G / H}(D)\right) x^{s}$.

Let $0 \neq f x \in H^{0}(X / H, \mathscr{M}(\alpha))$. Then

$$
\begin{aligned}
(f x)^{s} & =(f x) \otimes(f x)^{\sigma} \otimes \ldots \otimes(f x)^{\sigma^{s-1}} \\
& =\left(f f^{\sigma} \ldots f^{\sigma^{s-1}}\right) x \otimes x^{\sigma} \otimes \ldots \otimes x^{\sigma^{s-1}}=\left(f f^{\sigma} \ldots f^{\sigma^{s-1}}\right) x^{s} .
\end{aligned}
$$

But $\operatorname{Gal}\left(k(E) / k\left(E^{\prime}\right)\right)=G / H$ so $(f x)^{s}=(N f) x^{s}$ where $N: k(X / H) \rightarrow k(X / G)$ is the norm map. Hence, by $(2.10) f x \rightarrow(f x)^{s}$ is surjective. 
The last two results of this section treat the case relevant to the Sklyanin algebra. Thus $X=E$ will be an elliptic curve, $\sigma$ will be translation by a point of order $n=r s$, $\mathscr{L}$ will be of degree $d \geqslant 3$, and we assume that $r$ divides $d$ and is prime to the characteristic of $k$. These hypotheses apply in (2.12) and (2.13). In particular $\left(\sigma^{s}\right)^{d}=1$ whence $\mathscr{L}^{\sigma^{s}} \cong \mathscr{L}$.

DEFINITION. We say that $0 \neq x \in B_{1}$ is good if its divisor of zeroes $D:=(x)_{0}$ has the following property: each point in $\operatorname{Supp} D$ occurs with multiplicity $1, D^{\sigma^{s}}=D$, and $\operatorname{Supp}(D) \cap \operatorname{Supp}\left(D^{\sigma j}\right)=\emptyset$ for $0<j<s$.

If $x$ is good then

$$
\left(x^{\rho}\right)=\left(u\left(x^{\sigma^{s}}\right)\right)=\left(x^{\sigma^{s}}\right)=(x)^{\sigma^{-s}}=(x),
$$

so $x$ is a $\rho$-eigenvector. Furthermore, if $x^{\rho}=\zeta^{-1} x$ then $\zeta^{r}=1$, so we may replace $u$ by $u^{\prime}=\zeta u$ and the $\rho^{\prime}$ determined by $u^{\prime}$ is $\lambda_{\zeta}{ }^{\circ} \rho$, and $x^{\rho^{\prime}}=x$. Given a good $x$ we will frequently choose a $\rho$ such that $x$ is $\rho$-invariant.

PROPOSITION 2.12. There is a basis $\left\{x_{1}, \ldots, x_{d}\right\}$ for $B(E, \sigma, \mathscr{L})_{1}$ such that

(a) each $x_{i}$ is good, and

(b) $\left\{x_{1}^{s}, \ldots, x_{d}^{s}\right\}$ is a basis for $B(E, \sigma, \mathscr{L})_{s}^{G^{\prime}}$.

Proof. For each $\alpha \in \hat{H}$ set

$$
B_{1}(\alpha)=H^{0}(E / H, \mathscr{M}(\alpha)) \quad \text { and } \quad B_{s}^{G^{\prime}}(\alpha)=H^{0}\left(E / G, \mathscr{L}^{\prime \prime}(\alpha)\right) .
$$

The preceding discussion gives decompositions

$$
B_{1}=\bigoplus_{\alpha \in \hat{\Pi}} B_{1}(\alpha), \quad B_{s}^{G^{\prime}}=\bigoplus_{\alpha \in\left(G / G^{\prime}\right)^{\prime}} B_{s}^{G^{\prime}}(\alpha)
$$

such that $x \rightarrow x^{s}$ is a surjective morphism $B_{1}(\alpha) \rightarrow B_{s}^{G^{\prime}}(\alpha)$. It suffices to prove that for each $\alpha \in \hat{H}$, there is a basis $\left\{x_{1}, \ldots, x_{c}\right\}$ for $B_{1}(\alpha)$ such that each $x_{i}$ is good, and $\left\{x_{1}^{s}, \ldots, x_{c}^{s}\right\}$ is a basis for $B_{s}^{G^{\prime}}(\alpha)$. We observe that $\mathscr{M}(\alpha)$ and $\mathscr{L}^{\prime \prime}(\alpha)$ have the same degree and, hence, $B_{1}(\alpha)$ and $B_{s}^{G^{\prime}}(\alpha)$ have the same dimension.

Suppose that $0 \neq x \in B_{1}(\alpha)$. Let $D=(x)$. Since $\rho(x)$ is a scalar multiple of $x$, it has the same divisor as $x$. But $\rho(x)=u\left(x^{\sigma^{s}}\right)$, so $(\rho(x))=\left(x^{\sigma^{s}}\right)=(x)^{\sigma^{-s}}$. Thus $D^{\sigma^{s}}=D$. Furthermore $\left\{0 \neq x \in B_{1}(\alpha) \mid(x) \cap(x)^{\sigma^{j}}=\emptyset\right.$ for all $\left.0<j<s\right\}$ is Zariski open in $B_{1}(\alpha)$. Hence, $\left\{x \in B_{1}(\alpha) \mid x\right.$ is good $\}$ is Zariski open in $B_{1}(\alpha)$. Since the map $x \rightarrow x^{s}$ is surjective the image of a dense subset of $B_{1}(\alpha)$ is dense in $B_{s}^{G^{\prime}}(\alpha)$. In particular the image of this map cannot be contained in a proper subspace of $B_{s}^{G^{\prime}}(\alpha)$. Hence, the image of the morphism $\varphi: B_{1}(\alpha)^{c} \rightarrow B_{s}^{G^{\prime}}(\alpha)^{c}$ given by $\varphi\left(x_{1}, \ldots, x_{c}\right)=\left(x_{1}^{s}, \ldots, x_{c}^{s}\right)$ contains some linearly independent element. However, the linearly independent elements of $B_{s}^{G^{\prime}}(\alpha)^{c}$ form a Zariski open set, and hence their preimages in $B_{1}(\alpha)$ form a nonempty Zariski open set. But

$$
\left\{\left(x_{1}, \ldots, x_{c}\right) \in B_{1}(\alpha)^{c} \mid \text { each } x_{i} \text { is good, and }\left\{x_{1}, \ldots, x_{c}\right\} \text { is linearly independent }\right\}
$$

is also nonempty and open. The intersection of these two sets therefore contains an element which satisfies the proposition. 
DEFINITION. A good basis for $B_{1}$ is one satisfying the conditions of (2.12).

COROLLARY 2.13. Let $x_{1}, \ldots, x_{d}$ be a good basis for $B(E, \sigma, \mathscr{L})_{1}$. Then

$$
\begin{aligned}
B(E, \sigma, \mathscr{L}) & =k\left[x_{1}, \ldots, x_{d}\right], \\
B\left(E / G^{\prime}, \sigma^{s}, \mathscr{L}^{\prime}\right) & =k\left[x_{1}^{s}, \ldots, x_{d}^{s}\right], \\
Z\left(B(E, \sigma, \mathscr{L})^{(r)}\right) & =k\left[x_{1}^{s}, \ldots, x_{d}^{s}\right]^{(n)} .
\end{aligned}
$$

Furthermore, if $x_{i}^{\rho}=\zeta^{-1} x_{i}$ then $x_{i}^{s}$ is $\left(\lambda_{\zeta} \circ \rho\right)$-normalizing, where $\lambda_{\xi}$ is the automorphism acting as scalar multiplication by $\zeta^{j}$ on degree $j$ elements.

We remind the reader that we always take account of the original degrees of elements: for example, $x_{1}^{n} \in k\left[x_{1}^{s}, \ldots, x_{i}^{s}\right]^{(n)}$.

Proof. Since $\operatorname{deg}\left(\mathscr{L}^{\prime}\right)=\operatorname{deg}(\mathscr{L}) \geqslant 3$, both $B(E, \sigma, \mathscr{L})$ and $B\left(E / G^{\prime}, \sigma^{s}, \mathscr{L}^{\prime}\right)$ are generated in degee 1. Hence the equalities follow from (2.8) and (2.12).

Now suppose that $x=x_{i}$ has $\rho$-eigenvalue $\zeta^{-1}$. If $y \in B(E, \sigma, \mathscr{L})_{1}$ then $y^{p} x^{s}=$ $\left(x^{s}\right)^{\tau} y=\zeta^{-1} x^{s} y$ where the equalities follow from (2.2d) and (2.2a), respectively.

\section{Generators of $Z(A)$ and $Z\left(A^{(a)}\right)$}

We consider the situation relevant to the Sklyanin algebra, namely $X=E$ is an elliptic curve, $\sigma$ is translation by a point of order $n=r s$ and $\mathscr{L}$ is of degree $d \geqslant 3$. Using (2.1) we will identify $B(E, \sigma, \mathscr{L})$ with a subalgebra of its localization $B\left(\xi, \sigma, \mathscr{L}_{\xi}\right)$ which is isomorphic to the Ore extension $K\left[t, t^{-1} ; \sigma\right]$. Since the action of $\sigma$ on $K$ agrees with conjugation by $t$, we extend $\sigma$ to an automorphism of $K\left[t, t^{-1} ; \sigma\right]$ by declaring $t^{\sigma}=t$.

LEMMA 3.1. Fix an arbitrary factorization $n=r s$. Let $\delta$ be a $\sigma^{s}$-derivation of $K\left[t, t^{-1} ; \sigma\right]$ of degree $l$ such that $s-l$ is not divisible by $n$. Then there exists $f \in K$ such that $\delta$ is the inner $\sigma^{s}$-derivation by $f t^{l}$. If $\delta(t)=0$ then $f \in K^{G}$.

Proof. We must show that there is an $f \in K$ such that

(1) $\delta(a)=f\left(a^{a^{l}}-a^{a^{s}}\right) t^{l}$, for all $a \in K$, and

(2) $\delta(t)=\left(f-f^{\sigma}\right) t^{l+1}$.

Define $\phi: K \rightarrow K$ by $\delta(a)=\phi(a) t^{l}$. Then using the product rule to expand each side of the equation $\delta(a b)=d(b a)$, we find on choosing $b \in K$ such that $b^{\sigma^{l}} \neq b^{\sigma^{s}}$ that (1) holds with $f=\phi(b) /\left(b^{\sigma^{l}}-b^{\sigma^{s}}\right)$. Define $g \in K$ by $\delta(t)=g t^{l+1}$ and use the product rule on $\delta(t b)=\delta\left(b^{\sigma} t\right)$ to get $(2)$.

LEMMA 3.2. Let $D$ be a divisor of degree $\geqslant 3$ and let $V=H^{0}\left(E, \mathscr{O}_{E}(D)\right)$. For an arbitrary divisor $D^{\prime}$, and a point $p \in E$, let $m\left(p, D^{\prime}\right)$ denote the multiplicity of $p$ in $D^{\prime}$. With $l$ and $s$ as in Lemma 3.1, we have, for each $p \in E$,

$$
\inf \left\{m\left(p,\left(w^{\sigma^{1}}-w^{\sigma^{s}}\right)\right) \mid w \in V\right\}=-\max \left\{m\left(p, D^{\sigma^{-1}}\right), m\left(p, D^{\sigma^{-s}}\right)\right\} .
$$

Proof. By symmetry (i.e., interchanging $l$ and $s$ if necessary) we can suppose that $m\left(p, D^{\sigma^{-l}}\right) \leqslant m\left(p, D^{\sigma^{-s}}\right)$. Note that since $\sigma^{s}$ and $\sigma^{l}$ are unequal translations, $p^{\sigma^{s}} \neq p^{\sigma^{l}}$ 
for each $p$. Since $\operatorname{deg}(D) \geqslant 3$, there is a $w \in V$ such that $m\left(p,\left(w^{\sigma^{l}}\right)\right)>-m\left(p, D^{\sigma^{-i}}\right)$ but $m\left(p,\left(w^{\sigma^{s}}\right)\right)=-m\left(p, D^{\sigma-s}\right)$. That $w$ shows that the infimum is less than of equal to the maximum. The other direction is obvious.

THEOREM 3.3. Fix an arbitrary factorization $n=r s$ with $r \mid d$. Suppose that $x \in B(E, \sigma, \mathscr{L})_{1}$ is good. Choose $\rho$ such that $x^{\rho}=x$. Let $\delta$ be a $\rho$-derivation of $B(E, \sigma, \mathscr{L})$ of degree $l$ such that $\delta(x)=0$.

(a) If $l \leqslant 0$ then $\delta=0$.

(b) If $0<l<n$ then $\delta$ is an inner derivation by $c x^{l}$ for some $c \in k$.

Proof. Extend $\delta$ to the central localization $B\left(\xi, \sigma, \mathscr{L}_{\xi}\right)$. Identify $B\left(\xi, \sigma, \mathscr{L}_{\xi}\right)$ with $K\left[t, t^{-1} ; \sigma\right]$ via the isomorphism sending $x$ to $t$. Thus, both $\rho$ and $\delta$ extend to $K\left[t, t^{-1} ; \sigma\right]$ via this identification. Recall the definition prior to (3.1) of the automorphism $\sigma$ of $K\left[t, t^{-1} ; \sigma\right]$. Since the restriction of $\rho$ to $K$ agrees with $\sigma^{s}$ and since $x^{\rho}=x$ implies that $t^{\rho}=t=t^{\sigma^{s}}$, it follows that $\rho=\sigma^{s}$. Thus $\delta$ is a $\sigma^{s}$-derivation of $K\left[t, t^{-1} ; \sigma\right]$.

Set $D=(x)$ and $V=\mathcal{O}_{E}(D)$, whence $B_{1}=V t$.

(Case 1: $s=n, \rho=1, \delta$ a derivation.)

If $l>0$, then, by Lemma $3.1, \delta$ is inner by $f t^{l}$ for some $f \in K^{G}$. We must show $f$ has no pole. Since $\delta(B) \subset B$ we have $\delta\left(B_{1}\right) \subset B_{l+1}$. By a trivial calculation $f\left(w^{\sigma^{l}}-w\right) \in H^{0}\left(E, \mathcal{O}\left(D+D^{\sigma^{-1}}+\cdots+D^{\sigma^{-1}}\right)\right)$ for all $w \in V$. Therefore by Lemma 3.2, for each point $q \in E / G$, we have

$$
m(q,(f)) \geqslant \max \left\{m\left(p, D^{-\sigma^{x}}\right), m(p, D)\right\}-S,
$$

for each $p$ above $q$, where $S$ is the sum of the numbers $m\left(p, D^{-\sigma^{i}}\right)$ for $0 \leqslant i \leqslant l$. Since $x$ is good, at most one of these numbers is not 0 . Choose $p$ above $q$ so that the nonzero one is $m(p, D)$, and find

$$
m(q,(f)) \geqslant m(p, D)-m(p, D)=0 .
$$

If $l=0$, then $\left.\delta\right|_{K}$ is a $k$-derivation of $K$ such that $\delta(V) \subset V$. We may write $\delta=g(d / \omega)$, where $\omega$ is a nonzero differential of the first kind, and $g \in K$. Let $p$ be a point of $E$. If $m(p, D)=0$, choose $f \in V$ such that $m(p,(f))=1$. Then $m(p,(d f / \omega))=0$, so $g$ has no pole at $p$. If $m(p, D)=m>0$, choose $f \in V$ such that $m(p, f)=-m$. Then $m(p,(d f / \omega))=-m-1$, so $g$ has a zero at $p$ unless $m$ is divisible by the characteristic of $k$; but $x$ is good, so $D$ does not have a multiple point, i.e. $m=1$. So $g$ has zeros but no poles whence $\delta=0$, as was to be shown.

If $l=-1$, then by Lemma 3.1, we have, as in case $l>0$ above,

$$
m(q,(f)) \geqslant \max \left\{m\left(p, D^{-\sigma^{x}}\right), m(p, D)\right\},
$$

for each $p$ above $q$, (the sum $S$ has no terms, so is 0 ), so $f$ has zeros but no poles. If $l \leqslant-2$, then $\delta$ is trivially 0 .

(Case 2: $s<n, \rho$ of order $r>1$.) 
Suppose that $l-s$ is divisible by $n$. This happens for an $l \geqslant-1$ only if $l=-1, s=1$, and $n=2$. We settle that case. We have $\rho=\sigma$, of order 2 , so $\delta$ is a $\sigma$-derivation taking $V$ to $k$. Define the $k$-linear map $\phi: K \rightarrow K$ by $\delta(a t)=\sigma(\phi(a))$. Then $\phi$ is a $k$-derivation of $K$ taking $V$ to $k$, so taking $V$ into $V$, and we have seen that the only such derivation is the zero derivation.

In all other cases, Lemmas 3.1 and 3.2 apply, as in Case 1.

We are almost ready to lift the generators of $Z(B(E, \sigma, \mathscr{L}))$ and $Z\left(B(E, \sigma, \mathscr{L})^{(r)}\right)$ up to $A$ and to obtain generators of $Z(A)$ and $Z\left(A^{(r)}\right)$.

Recall that $a=6-d$ denotes the degree of the central elements $g_{1}, \ldots, g_{m}$.

LEMMA 3.4.

(a) There is a unique lifting of $\rho$ to an automorphism of $A$.

(b) Suppose that $r \mid a$. Each of the central elements $g_{1}, \ldots, g_{m}$ is $\rho$-invariant.

Proof. (a) Since the map $A \rightarrow B$ is an isomorphism in degree 1 there is at most one lifting of $\rho$ to $A$. Since $a$ is prime, the only case to consider is $r=a$, because $\rho=1$ if $r=1$.

Let $\rho$ also denote the automorphism of $\mathbb{P}\left(A_{1}^{*}\right)$ given by

$$
\rho(x)(p)=x\left(p^{\rho}\right) \text { for } x \in A_{1} \quad \text { and } \quad p \in \mathbb{P}\left(A_{1}^{*}\right) .
$$

If $p \in E \subset \mathbb{P}\left(A_{1}^{*}\right)$, then $\rho(x)=u\left(x^{\sigma^{s}}\right)(p)=x\left(p^{\sigma^{s}}\right)$ so $\left.\rho\right|_{E}=\sigma^{s}$. Since $\rho^{a}=1$ this is translation by an $a$-torsion point $\omega \in E$.

Now let $f \in R_{A} \subset A_{1} \otimes A_{1}$. We must show that $(\rho \otimes \rho)(f) \in R_{A}$. If $p \in E$ then

$$
(\rho \otimes \rho)(f)\left(p, p^{\sigma}\right)=f\left(p^{\sigma^{s}}, p^{\sigma^{s+1}}\right)=0 .
$$

This proves the result for $\mathrm{d}=3$. Now suppose that $d=4$, and consider $(\rho \otimes \rho)(f)\left(e_{i}, e_{i}\right)$, where $e_{i}$ is the vertex of one of the quadric cones containing $E$. By [10] these 4 points may be characterized as the only points of $\mathbb{P}^{3} \backslash E$ which lie on infinitely many secant lines to $E$. Furthermore, for each $e_{i}$, there is a unique $\omega_{i} \in E_{2}$ such that $e_{i}$ lies on the secant line $l_{p q}$ through $p, q \in E$ if and only if $p+q=\omega_{i}$. The action of $\rho$ on $\mathbb{P}\left(A_{1}^{*}\right)$ sends the line $l_{p q}$ to $l_{p+\omega, q+\omega}$. Since $2 \omega=0$ this line passes through $e_{i}$ also. Therefore, $e_{i}^{\rho}=e_{i}$. Hence, $(\rho \otimes \rho)(f)\left(e_{i}, e_{i}\right)=0$ so $(\rho \otimes \rho)(f) \in R_{A}$.

(b) Suppose that $d=3$. Since $\rho$ is of order 3 and $k$ is algebraically closed of characteristic different from 3 we can choose basis elements $x, y, z$ for $A_{1}$ which are eigenvectors for $\rho$. As is well known, the eigenvalues are distinct (if, for example, $\rho(x / y)=x / y$, then $\rho=\sigma^{s}$ would fix the points on $E$ where $z=0$, contradicting the fact that $\sigma^{s}$ is a translation). So we can suppose that $\rho(x)=x, \rho(y)=\zeta y, \rho(z)=\zeta^{2} z$, where $\zeta$ is a primitive third root of 1 . The cubic equation $f(x, y, z)=0$ defining $E$ is preserved by $\rho$, so must be of one of the three forms

$$
\begin{aligned}
& a x^{3}+b y^{3}+c z^{3}+d x y z=0, \quad a x^{2} y+b y^{2} z+c z^{2} x=0, \quad \text { or } \\
& a x y^{2}+b y z^{2}+c z x^{2}=0
\end{aligned}
$$


for some $a, b, c, d \in k$. The last two are not possible, because then $(1,0,0) \in E$ would be a fixed point for $\sigma^{s}$, and for the same reason, the coefficients $a, b, c$ in the first equation are not zero. Therefore, after scaling $x, y, z$, we can assume that the cubic defining $E$ is in the 'canonical form' $f=x^{3}+y^{3}+z^{3}-m x y z$ and that $\rho(x)=x, \rho(y)=\zeta y, \rho(z)=\zeta^{2} z$. Let $(1,-1,0)^{\sigma}=(a, b, c)$.

As explained in the introduction to [4], the algebra $A$ is defined by the three relations $f_{i}(x, y, z)=0$, where

$$
\begin{aligned}
& f_{1}(X, Y, Z):=a Y Z+b Z Y+c X^{2}, \\
& f_{2}(X, Y, Z):=a Z X+b X Z+c Y^{2}, \\
& f_{3}(X, Y, Z):=a X Y+b Y X+c Z^{2} .
\end{aligned}
$$

(Strictly speaking, the argument in [4] applies only in case $\sigma$ is not of order dividing 3 , in which case $a b c \neq 0$ and the equation for $E$ can be written

$$
a b c\left(x^{3}+y^{3}+z^{3}\right)=\left(a^{3}+b^{3}+c^{3}\right) x y z .
$$

However, it is not hard to see that the set of points $(a, b, c) \in E$ for which those relations define $A$ is closed, and, consequently, they are the relations also if $\sigma^{3}=1$.)

Let $T=T\left(A_{1}\right)=k\langle X, Y, Z\rangle$ be the tensor algebra on $A_{1}$, with $X, Y, Z$ denoting the elements of $T_{1}$ whose images in $A$ are $x, y, z$. Let $A=T / J$. Then each of the 3-dimensional spaces

$$
T_{1}=k X+k Y+k Z \text { and } J_{2}=k f_{1}(X, Y, Z)+k f_{2}(X, Y, Z)+k f_{3}(X, Y, Z)
$$

is isomorphic, as a representation of the group $\langle\rho\rangle$, to the regular representation. Since $A_{3}=T_{3} / J_{3}$ is of dimension 10 , it follows that $\operatorname{dim}\left(J_{3}\right)=17$. But $J_{3}$ is the sum of the two 9-dimensional spaces $T_{1} J_{2}$ and $J_{2} T_{1}$. Hence these spaces intersect in a 1-dimensional space. That space contains the element

$$
X f_{1}+Y f_{2}+Z f_{3}=f_{1} X+f_{2} Y+f_{3} Z
$$

which is fixed by $\rho$. It follows that the trivial representation of $\langle\rho\rangle$ occurs with multiplicity $3+3-1=5$ in $J_{3}$, and therefore with multiplicity $9-5=4$ in $A_{3}$. On the other hand, it occurs only 3 times in $B_{3}$, by Proposition 2.5 applied to the $\langle\rho\rangle$-triple $(E, \sigma, \mathscr{L})$, because the descent of $\mathscr{L}$ to $E /\left\langle\sigma^{s}\right\rangle$ is of degree 1 . Since $B_{3}=A_{3} / \mathrm{kg}$, it follows that $g$ is fixed by $\rho$.

Suppose that $d=4$. As in [13] there is a basis $\left\{x_{0}, \ldots, x_{3}\right\}$ for $A_{1}$ such that the defining equations of $E$ are linear combinations of the $x_{i}^{2}$. Hence, the vertices $e_{0}, \ldots, e_{3}$ of the 4 quadric cones containing $E$ are defined by the vanishing of three of the $x_{i}$. Since $e_{j}^{\rho}=e_{j}$ each $x_{j}$ is a $\rho$-eigenvector. Since $\rho^{2}=1$, each $x_{j}^{2} \in A_{2}$ is $\rho$-invariant. Since the central elements $g_{1}$ and $g_{2}$ are linear combinations of the $x_{j}^{2}$,s $[13]$ they are also $\rho$-invariant.

Remarks. (1) According to $[2,10.17]$, the central element for the 3-dimensional case, when the generators and relations are as in the proof of (3.4), is

$$
g=\left(c^{3}-b^{3}\right)\left(c y^{3}-a x y z\right)+\left(c^{3}-a^{3}\right)\left(b y x z-c x^{3}\right),
$$


which is obviously fixed by $\rho$. But checking that this element is central (and not 0 ) seems not so easy.

(2) Actually the proof of (3.4) makes no use of the fact that $\sigma$ is of finite order, or of the relation $\rho=\sigma^{s}$ between $\rho$ and $\sigma$, except to deduce that $\rho$ is a translation. Hence we have really shown when $d=3$ that $g$ is fixed by every automorphism of $A$ which induces on $E$ a translation, that is, by the elements of the Heisenberg group of order 27 generated by the two automorphisms $(x, y, z) \rightarrow\left(x, \zeta y, \zeta^{2} z\right)$ and $(x, y, z) \rightarrow(y, z, x)$.

LEMMA 3.5. Suppose that $r \mid a$. Let $0 \neq x \in B(E, \sigma, \mathscr{L})_{1}$ be good, and choose $\rho$ such that $x^{\rho}=x$. Then there exists $u \in A_{s}$ which is $\rho$-normalizing in $A$, and is of the form

$$
u=x^{s}+\sum_{1 \leqslant j \leqslant \frac{s}{a}} c_{j} x^{s-j a}
$$

with each $c_{j} \in k\left[g_{1}, \ldots, g_{m}\right]_{a j}$.

We remind the reader that we always take account of the original degrees of elements: for example, $g_{1}^{j} \in k\left[g_{1}, \ldots, g_{m}\right]_{a j}$.

Proof. Write $J:=\left(g_{1}, \ldots, g_{m}\right)$, whence $B=A / J$. Since $g_{1}, \ldots, g_{m}$ is a regular sequence, for all $l \geqslant 1, J^{l} / J^{l+1}$ is a free $B$-module with basis the monomials in $g_{1}, \ldots, g_{m}$ of degree al.

By (2.13) $x^{s}$ is $\rho$-normalizing in $B$. Hence for all $y \in A, x^{s} y-y^{\rho} x^{s} \in J$. Passing to the image in $J / J^{2}$, viewed as a $B$-module with basis $\left\{g_{1}, \ldots, g_{m}\right\}$, we may write $x^{s} y-y^{\rho} x^{s}=\Sigma_{1 \leqslant i \leqslant m} \psi_{i}(y) g_{i}$ for unique elements $\psi_{i}(y) \in B$.

If $y \in A$, write $\bar{y}$ for the image of $y$ in $B$. Claim: The maps $\delta_{i}: B \rightarrow B$ defined by $\delta_{i}(\bar{y})=\psi_{i}(y)$ are $\rho$-derivations of degree $s-a$, with the property that $\delta_{i}(x)=0$.

Proof: To see that $\delta_{i}$ is well-defined, suppose that $\bar{y}=\bar{w}$. Thus $w-y=\Sigma_{i} a_{i} g_{i}$ for some $a_{i} \in A$. Therefore $x^{s}(w-y)-(w-y)^{\rho} x^{s}=\Sigma_{i}\left(x^{s} a_{i}-a_{i}^{\rho} x^{s}\right) g_{i} \in J^{2}$ where we have used the $\rho$-invariance of the $g_{i}$. It follows that $\psi_{i}(w-y)=0$ for all $i$ whence $\delta_{i}(\bar{w})=\delta_{i}(\bar{y})$. A straightforward calculation shows that $\delta_{i}$ is a $\rho$-derivation. Since $x$ commutes with $x^{s}$, we have $\delta_{i}(x)=0$ for all $i$. This proves the claim.

Hence by (3.3) with $l=s-a, \delta_{i}$ is an inner $\rho$-derivation by $\alpha_{i} x^{s-a}$ for some $\alpha_{i} \in k$. Therefore if we define $c_{1}=-\Sigma_{1 \leqslant i \leqslant m} \alpha_{i} g_{i} \in k\left[g_{1}, \ldots, g_{m}\right]_{a}$ we obtain $\left(x^{s}+c_{1} x^{s-a}\right) y-$ $y^{\rho}\left(x^{s}+c_{1} x^{s-a}\right) \in J^{2}$ for all $y \in A$.

Now we proceed by induction in the same fashion, using the fact that each $J^{l} / J^{l+1}$ is a free $B$-module with basis the monomials in $g_{1}, \ldots, g_{m}$ of degree $l a$. At this stage, one obtains $\rho$-derivations of $B$ of degree $s-l a$. However, if $s-l a \leqslant 0$ such a derivation is zero by (3.3a), so eventually we obtain an element $u$ of the prescribed form such that $u y-y^{\rho} u \in J^{m}$ for all $m \gg 0$, and hence $u y=y^{\rho} u$ as desired. This completes the proof of the lemma.

LEMMA 3.6. Let $R$ be an $\mathbb{N}$-graded $k$-algebra, and $g_{1}, \ldots, g_{m}$ a regular sequence of homogeneous central elements of positive degree. Set $\bar{R}:=R /\left(g_{1}, \ldots, g_{m}\right)$. Let $Z$ be a 
graded subalgebra of $Z(R)$ and let $\bar{Z}$ be its image in $\bar{R}$. Suppose that $\bar{Z}=Z(\bar{R})$. Then

(a) $Z(R)=Z\left[g_{1}, \ldots, g_{m}\right]$

(b) if $\bar{R}$ is finite over its center, $R$ is finite over its center;

(c) $Z(R) \cap\left(g_{1} R+\cdots+g_{m} R\right)=g_{1} Z(R)+\cdots+g_{m} Z(R)$.

Proof. If (a) is true for $m=1$, then repeated application of (a) establishes its truth for $m>1$. The same comment applies to (b). Hence to prove (a) and (b) we assume that we have a single regular homogeneous central element $g$ of degree $d>0$.

(a) We show by induction on $i$ that $Z(R)_{i} \subset Z[g]_{i}$. This is true for $i=0$. Let $i>0$ and let $a \in Z(R)_{i}$. Since $\overline{Z(R)} \subset Z(\bar{R}) \subset \bar{Z}$ there is an element $z \in Z_{i}$ such that $a=z+b g$ for some $b \in R_{i-d}$. Now $a-z=b g \in Z(R)$ and hence $b \in Z(R)$ as $g$ is not a zero-divisor. Since $\operatorname{deg}(b)<i$, we have $b \in Z[g]$ by the induction hypothesis. Thus $a=z+b g \in Z[g]$ as was to be shown.

(b) Suppose that the images of $\left\{a_{i} \mid i \in I\right\}$ generate $\bar{R}$ over its center. Without loss of generality we may suppose that all the $a_{i}$ are homogeneous. Let $f \in R_{j}$. We shall show by induction on $j$ that $f$ is in $\Sigma_{i} Z(R) a_{i}$. Write $f=\Sigma_{i} z_{i} a_{i}+g b$ with $z_{i} \in Z(R)$ and $b \in R_{j-d}$. The result follows from the induction hypothesis applied to $b$.

(c) We proceed by induction on $m$. If $m=1$ and $g_{1} a \in Z(R)$, then certainly $a \in Z(R)$ since $g_{1}$ is regular. Suppose that (c) is true for $m-1$.

Let $a_{i} \in R$ be such that $\Sigma_{i} g_{i} a_{i} \in Z(R)$. Let $\varphi: R \rightarrow R / g_{1} R$ be the natural map. Since

$$
\sum_{i=2}^{m} \varphi\left(g_{1}\right) \varphi\left(a_{i}\right) \in Z\left(R / g_{1} R\right)
$$

the induction hypothesis applied to $R / g_{1} R+\cdots+g_{m} R$ as a quotient of $R / g_{1} R$ gives

$$
\sum_{i=2}^{m} \varphi\left(g_{1}\right) \varphi\left(a_{i}\right)=\sum_{i=2}^{m} \varphi\left(g_{1}\right) \varphi\left(b_{i}\right)
$$

for some $b_{i} \in R$ such that $\varphi\left(b_{i}\right) \in Z\left(R / g_{1} R\right)$. However, by (a)

$$
Z\left(R / g_{1} R\right)=\varphi(Z)\left[\varphi\left(g_{2}\right), \ldots, \varphi\left(g_{m}\right)\right] .
$$

Hence, there exist elements $c_{i} \in Z\left[g_{1}, \ldots, g_{m}\right]$ and $d_{i} \in R$ such that $b_{i}=c_{i}+g_{1} d_{i}$. It follows that

$$
\sum_{i=2}^{m} g_{i} a_{i}=\sum_{i=2}^{m} g_{i}\left(c_{i}+g_{1} d_{i}\right) \bmod \left(g_{1} R\right)
$$

whence

$$
\sum_{i=1}^{m} g_{i} a_{i}=\sum_{i=2}^{m} g_{i} c_{i}+g_{1} u
$$

for some $u \in R$. Since $c_{i} \in Z(R)$ it follows from the regularity of $g_{1}$ that $u \in Z(R)$ also. Hence, $\Sigma_{i} g_{i} a_{i} \in g_{1} Z(R)+\cdots+g_{m} Z(R)$ as required. 
We emphasize that there are two cases in the next theorem, namely $r=a$ and $r=1$, the second of which gives generators for $Z(A)$.

THEOREM 3.7. Let $A$ be either the 3-dimensional or 4-dimensional Sklyanin algebra. Suppose that $r \mid a$. Let $x_{1}, \ldots, x_{d}$ be a good basis for $A_{1}$. Then $Z\left(A^{(r)}\right)=$ $k\left[u_{1}, \ldots, u_{d}\right]^{(n)}\left[g_{1}, \ldots, g_{m}\right]$ where

(a) each $u_{i}$ is of the form

$$
u_{i}=x_{i}^{s}+\sum_{1 \leqslant j \leqslant \frac{s}{r}} c_{i j} x_{i}^{s-j r}
$$

for some $c_{i j} \in k\left[g_{1}, \ldots, g_{m}\right]_{r j}$;

(b) If $x_{i}^{\rho}=\zeta^{-1} x$ then $u_{i}$ is $\left(\lambda_{\zeta} \circ \rho\right)$-normalizing in $A$;

(c) $A$ is a finite module over its center.

Proof. (a) (b) Since $x_{i}$ is $\left(\lambda_{\zeta^{\circ}} \rho\right)$-invariant, (3.5) yields elements $u_{i}$ of the prescribed form which are $\left(\lambda_{\zeta} \circ \rho\right)$-normalizing in $A$. Set $Z=k\left[u_{1}, \ldots, u_{d}\right]^{(n)}$ and $R=A^{(r)}$. Since $r \mid a$ each $g_{j} \in R$. Under the natural map $A \rightarrow B$ the image of $R$ is $B^{(r)}$ and the image of $Z$ is $Z\left(B^{(r)}\right)$ by (2.13). Since

$$
B^{(r)}=A^{(r)} / A^{(r)} \cap\left(A g_{1}+\cdots+A g_{m}\right)=R / R g_{1}+\cdots+R g_{m}
$$

we may apply (3.6a) to conclude that $Z(R)=Z\left[g_{1}, \ldots, g_{m}\right]$ as required.

(c) This follows from (3.6b) and (2.3) in the case $r=1$.

Notation. Recall that $b=\operatorname{gcd}(a, n)$. After (4.2) we will reserve the notation $u_{1}, \ldots, u_{d}$ for the elements of degree $n / b$ which are obtained in (3.7) when $r=b$, and will use the notation $z_{i}$ for the $u_{i}$ obtained in (3.7) when $r=1$. Thus

$$
Z(A)=k\left[z_{1}, \ldots, z_{d}\right]\left[g_{1}, \ldots, g_{m}\right]
$$

and if $b=1$ then $u_{i}=z_{i}$. We will show in (4.6) that we may take $z_{i}=u_{i}^{b}$ in general: we can't do this yet since we don't know that $u_{1}^{b}, \ldots, u_{d}^{b}$ are linearly independent.

\section{The Relations in $Z(A)$ and $Z\left(A^{(b)}\right)$}

From now on we dispense with generalities and restrict our attention to the Sklyanin algebras.

Given Theorem 3.7, the first step towards obtaining defining relations for $Z(A)$ and $Z\left(A^{(b)}\right)$ is to determine defining relations for $k\left[u_{1}, \ldots, u_{d}\right]$. We do this by simultaneously giving a more functorial description of $k\left[u_{1}, \ldots, u_{d}\right]$. We will show it is isomorphic to a twist of a polynomial ring, and using the isomorphism in (4.1), this twisted ring has a good description in terms of the original data $(E, \sigma, \mathscr{L})$.

PROPOSITION 4.1. Consider a triple $\left(\mathbb{P}^{n}, \sigma, \mathcal{O}(1)\right)$. Let $w: \mathcal{O}(1)^{\sigma} \stackrel{\sim}{\longrightarrow} \mathcal{O}(1)$ be an isomorphism, and let $\theta \in \operatorname{Aut} B\left(\mathbb{P}^{n}, 1, \mathcal{O}(1)\right)$ be determined by $(\sigma, w) \in \operatorname{Aut}\left(\mathbb{P}^{n}, 1, \mathcal{O}(1)\right)$. Then the identity map $B\left(\mathbb{P}^{n}, 1, \mathcal{O}(1)\right)_{1} \rightarrow B\left(\mathbb{P}^{n}, \sigma, \mathcal{O}(1)\right)_{1}$ extends to an algebra iso- 
morphism

$$
\varphi: B\left(\mathbb{P}^{n}, 1, \mathcal{O}(1)\right)^{\theta} \stackrel{\sim}{\longrightarrow} B\left(\mathbb{P}^{n}, \sigma, \mathcal{O}(1)\right) .
$$

If $\sigma^{m}=1$ then $w$ may be chosen such that $\theta^{m}=1$.

Proof. Since $B\left(\mathbb{P}^{n}, 1, \mathcal{O}(1)\right)$ is a polynomial ring, its twist $B\left(\mathbb{P}^{n}, 1, \mathcal{O}(1)\right)^{\theta}$ is generated in degree 1 and its ideal of relations is generated by the relations of degree 2 [17]. If $x, y \in B\left(\mathbb{P}^{n}, 1, \mathcal{O}(1)\right)_{1}^{\theta}$ then

$$
x^{\theta} * y=x^{\theta} y^{\theta}=y^{\theta} x^{\theta}=y^{\theta} * x .
$$

Letting $x$ and $y$ run through a basis for $B\left(\mathbb{P}^{n}, 1, \mathcal{O}(1)\right)$ we obtain $\left(\begin{array}{c}n+1 \\ 2\end{array}\right)$ linearly independent relations of the form $x^{\theta} \otimes y-y^{\theta} \otimes x$. Hence, these are defining relations for $B\left(\mathbb{P}^{n}, 1, \mathcal{O}(1)\right)^{\theta}$.

Notice that $(\sigma, w) \in \operatorname{Aut}\left(\mathbb{P}^{n}, \sigma, \mathscr{O}(1)\right)$ also, and as such it determines $\rho \in$ Aut $B\left(\mathbb{P}^{n}, \sigma, \mathcal{O}(1)\right)$. Furthermore, $\rho$ and $\theta$ agree on

$$
B\left(\mathbb{P}^{n}, 1, \mathcal{O}(1)\right)_{1}^{\theta}=H^{0}\left(\mathbb{P}^{n}, \mathcal{O}(1)\right)=B\left(\mathbb{P}^{n}, \sigma, \mathcal{O}(1)\right)_{1}
$$

since

$$
\theta(x)=w\left(x^{\sigma}\right)=\rho(x) \text { for } x \in H^{0}\left(\mathbb{P}^{n}, O(1)\right) .
$$

By (2.2a), if $x, y \in B\left(\mathbb{P}^{n}, \sigma, \mathcal{O}(1)\right)_{1}$ then $x^{\rho} y=y^{\rho} x$ so the identity map does indeed extend to an algebra homomorphism $\varphi$. Since $B\left(\mathbb{P}^{n}, \sigma, \mathscr{O}(1)\right)$ is generated in degree 1 , $\varphi$ is surjective, and since the two rings have the same Hilbert series, $\varphi$ is an isomorphism.

As in the discussion after (2.2) we may replace any particular $w$ by a suitable scalar multiple such that $\theta$ and $\sigma$ have the same order.

Remark. If we are in the situation considered in (2.2) then, by (2.2a) $x^{\rho} y=y^{\rho} x$ for $x, y \in B(X, \sigma, \mathscr{L})_{s}$. Hence the subalgebra of $B(X, \sigma, \mathscr{L})^{(s)}$ generated by the elements of degree $s$ is a quotient of a twist of a polynomial ring.

Define $\mathbb{P}^{\prime}=\mathbb{P}\left(H^{0}\left(E / G^{\prime}, \mathscr{L}^{\prime}\right)^{*}\right) \cong \mathbb{P}^{d-1}$ and let $j: E / G^{\prime} \rightarrow \mathbb{P}^{\prime}$ be the inclusion. Then $\mathscr{L}^{\prime}=j^{*} \mathcal{O}_{\mathbb{P}}(1)$ and we can make the identification

$$
H^{0}\left(\mathbb{P}^{\prime}, \mathscr{O}(1)\right)=H^{0}\left(E / G^{\prime}, \mathscr{L}^{\prime}\right)=B\left(E / G^{\prime}, \sigma^{s}, \mathscr{L}^{\prime}\right)_{s}^{G^{\prime}} .
$$

The resulting action of $\rho$ on $H^{0}\left(\mathbb{P}^{\prime}, \mathcal{O}(1)\right)$ induces an automorphism $\mu$ of $\mathbb{P}^{\prime}$ which extends the automorphism $\sigma^{s}$ on $E^{\prime}$. Let $w: j^{*} \mathcal{O}(1) \rightarrow \mathscr{L}^{\prime}$ be the natural isomorphism. Then $(j, w):\left(E / G^{\prime}, \sigma^{s}, \mathscr{L}^{\prime}\right) \rightarrow\left(\mathbb{P}^{\prime}, \mu, \mathcal{O}(1)\right)$ is a morphism of triples, so determines an algebra homomorphism

$$
\alpha: B\left(\mathbb{P}^{\prime}, \mu, \mathcal{O}(1)\right) \rightarrow B\left(E / G^{\prime}, \sigma^{s}, \mathscr{L}^{\prime}\right) .
$$

PROPOSITION 4.2. Identify $B\left(E / G^{\prime}, \sigma^{s}, \mathscr{L}^{\prime}\right)$ with $B(E, \sigma, \mathscr{L})^{(s) G^{\prime}}$ as in (2.6). Let $\beta: k\left[u_{1}, \ldots, u_{d}\right] \rightarrow B\left(E / G^{\prime}, \sigma^{s}, \mathscr{L}^{\prime}\right)$ be the restriction of the surjection $A \rightarrow B$. There is a unique surjective algebra homomorphism

$$
\gamma: B\left(\mathbb{P}^{\prime}, \mu, \mathcal{O}(1)\right) \rightarrow k\left[u_{1}, \ldots, u_{d}\right]
$$

such that $\alpha=\beta \circ \gamma$. 
Proof. Suppose that the good basis $x_{1}, \ldots, x_{d}$ for $B(E, \sigma, \mathscr{L})_{1}$ satisfies $x_{i}^{\rho}=\zeta_{i}^{-1} x_{i}$. By $(3.7 \mathrm{~b}) u_{i}$ is $\left(\lambda_{\zeta_{i}} \circ \rho\right)$-normalizing, and therefore $\left(\lambda_{\zeta_{i}} \circ \rho\right)$-invariant. It follows that $u_{i} u_{j}=\left(\zeta_{i} \zeta_{j}^{-1}\right)^{s} u_{j} u_{i}$, or equivalently $u_{i}^{\rho} u_{j}=u_{j}^{\rho} u_{i}$. Therefore $u^{\rho} v=v^{\rho} u$ for all $u, v \in k\left[u_{1}, \ldots, u_{d}\right]_{s}$.

There exists $d: \mathcal{O}(1)^{\mu} \stackrel{\sim}{\longrightarrow} \mathcal{O}(1)$ such that the automorphism of $B\left(\mathbb{P}^{\prime}, \mu, \mathcal{O}(1)\right)$ induced by $(d, \mu)$ agrees in degree 1 with the action of $\rho$ on $H^{0}\left(\mathbb{P}^{\prime}, \mathcal{O}(1)\right)$. Hence $\rho$ extends to an automorphism of $B\left(\mathbb{P}^{\prime}, \mu, \mathcal{O}(1)\right)$.

The maps $\alpha$ and $\beta$ commute with the action of $\rho$ and are isomorphisms in degree 1 . Since $B\left(\mathbb{P}^{\prime}, \mu, \mathcal{O}(1)\right)$ is generated in degree 1 and has defining relations $x^{\rho} y=y^{\rho} x$ for $x, y$ of degree 1 , it follows that the map $\beta^{-1} \alpha$ in degree 1 extends to an algebra homomorphism $\gamma$.

Notation. Recall that $b=\operatorname{gcd}(a, n)$. From now on we will reserve the notation $u_{1}, \ldots, u_{d}$ for the elements of degree $n / b$ which arise in (3.7) with $r=b$. Thus $Z\left(A^{(b)}\right)=k\left[u_{1}, \ldots, u_{d}\right]^{(n)}\left[g_{1}, \ldots, g_{m}\right]$. Furthermore we will only use $s$ to denote $n / b$ and will no longer use $r$ to denote $n / s$. We will also write $E^{\prime}:=E /\left\langle\sigma^{b}\right\rangle=E /\left\langle\sigma^{a}\right\rangle$ and $E^{\prime \prime}:=E /\langle\sigma\rangle$. We will write $\mathscr{L}^{\prime}$ for the descent of $\mathscr{L}_{s}$ to $E^{\prime}$ and will write $\mathscr{L}^{\prime \prime}$ for the descent of $\mathscr{L}_{n}$ to $E^{\prime \prime}$. We will use $\mathbb{P}^{\prime}$ to denote $\mathbb{P}\left(H^{0}\left(E^{\prime}, \mathscr{L}^{\prime}\right)^{*}\right)$ and will use $\mathbb{P}^{\prime \prime}$ to denote $\mathbb{P}\left(H^{0}\left(E^{\prime \prime}, \mathscr{L}^{\prime \prime}\right)^{*}\right)$. We will write $j: E^{\prime} \rightarrow \mathbb{P}^{\prime}$ and $i: E^{\prime \prime} \rightarrow \mathbb{P}^{\prime \prime}$ for the two closed immersions. Hence, the two cases of (4.2) treat the $u_{i}$ as linear forms on $\mathbb{P}^{\prime}$, and the $z_{i}$ as linear forms on $\mathbb{P}^{\prime \prime}$. The notation $\mu$ and $\theta$ from (4.2) will only be applied to $k\left[u_{1}, \ldots, u_{d}\right]$.

Suppose $d=4$. We will denote the image in $E^{\prime}$ of $p \in E$ by $p^{\prime}$, and the image of $p-(s-1) \tau$ by $p^{\circ}$. If $p, q \in E$ we will write $l_{p q}$ for the secant line they span, and $l_{p^{\prime} q^{\prime}}$ will denote the secant line in $\mathbb{P}^{\prime}$ spanned by their images. The 2-torsion subgroup of $E$ will be denoted by $E_{2}$. The natural copy of $E$ in $\mathbb{P}\left(A_{1}^{*}\right)$ may have its identity located so that $p_{1}, p_{2}, p_{3}, p_{4} \in E$ are coplanar if and only if their sum is zero. We will assume this has been done (cf. [10]). In that case, the pencil of quadrics containing $E$ may be labelled $Q(z),(z \in E)$ so that $Q(z)=\cup\left\{l_{p q} \mid p+q= \pm z\right\}$. The 'same' is true of $j\left(E^{\prime}\right) \subset \mathbb{P}^{\prime}$, since if $x_{i}$ vanishes at $p_{1}, p_{2}, p_{3}, p_{4} \in E$ then $u_{i}$ vanishes at their images $p_{1}^{\prime}, p_{2}^{\prime}, p_{3}^{\prime}, p_{4}^{\prime} \in E^{\prime}$, and these sum to zero also.

Our next goal is to show that the map $\gamma: B\left(\mathbb{P}^{\prime}, \mu, \mathcal{O}_{\mathbb{P}}(1)\right) \rightarrow k\left[u_{1}, \ldots, u_{d}\right]$ obtained in (4.2) is an isomorphism. The idea for proving this is that if $k\left[u_{1}, \ldots, u_{d}\right]$ were a proper quotient of $B\left(\mathbb{P}^{\prime}, \mu, \mathcal{O}(1)\right)$ then its point modules would be parametrized by a proper subvariety of $\mathbb{P}^{\prime}$ and consequently its line modules would be parametrized by the lines lying on that subvariety. However, we will show that $k\left[u_{1}, \ldots, u_{d}\right]$ has too many line modules for this to happen. The line modules for $k\left[u_{1}, \ldots, u_{d}\right]$ will be realised as subspaces of line modules for $A$.

\section{BASES FOR LINE MODULES}

We begin by recalling that most line modules over the 3-dimensional and 4dimensional Sklyanin algebras have a nice basis. 
First let $d=4$. By [10, Proposition 5.6] if $p, q \in E$ are such that $p-q \notin 2 \mathbb{Z} \tau$ then the line module $M(p, q)$ corresponding to the secant line $l_{p q}$ has a basis $\left\{e_{i j} \mid i, j \geqslant 0\right\}$ such that

(a) $\operatorname{deg}\left(e_{i j}\right)=i+j$,

(b) $A e_{i j}[i+j] \cong M(p+(j-i) \tau, q+(i-j) \tau)$,

(c) $X . e_{i j} \in k e_{i+1, j}+k e_{i, j+1}$ for all $X \in A_{1}$,

(d) if $X \in A_{1}$ then $X . e_{i j} \in k e_{i+1, j}$ if and only if $X(q+(i-j) \tau)=0$,

(e) if $X \in A_{1}$ then $X \cdot e_{i j} \in k e_{i, j+1}$ if and only if $X(p+(j-i) \tau)=0$.

All these properties follow from the existence of the short exact sequence

$$
0 \rightarrow M(p+\tau, q-\tau)[-1] \rightarrow M(p, q) \rightarrow M(p) \rightarrow 0 .
$$

The element $e_{01}$ is chosen to be a generator of the kernel of the map $M(p, q) \rightarrow M(p)$.

Now take $d=3$. Although it is not stated explicitly there, the results in [5] show that there is a similar result for the 3-dimensional Sklyanin algebra. Suppose that $p-q \notin 3 \mathbb{Z} \tau$. There is a short exact sequence

$$
0 \rightarrow M(p+2 \tau, q-\tau)[-1] \rightarrow M(p, q) \rightarrow M(p) \rightarrow 0 .
$$

The element $e_{01}$ is chosen to be a generator of the kernel of the map $M(p, q) \rightarrow M(p)$ and $e_{10}$ is chosen to be a generator of the kernel of the map $M(p, q) \rightarrow M(q)$. Proceeding inductively, the line module $M(p, q)$ corresponding to the secant line $l_{p q}$ has a basis $\left\{e_{i j} \mid i, j \geqslant 0\right\}$ such that

(a) $\operatorname{deg}\left(e_{i j}\right)=i+j$,

(b) $A e_{i j}[i+j] \cong M(p+(2 j-i) \tau, q+(2 i-j) \tau)$,

(c) $X . e_{i j} \in k e_{i+1, j}+k e_{i, j+1}$ for all $X \in A_{1}$,

(d) if $X \in A_{1}$ then $X . e_{i j} \in k e_{i+1, j}$ if and only if $X(q+(2 i-j) \tau)=0$,

(e) if $X \in A_{1}$ then $X . e_{i j} \in k e_{i, j+1}$ if and only if $X(p+(2 j-i) \tau)=0$.

\section{ANNIHILATORS OF LINE MODULES}

For the 4-dimensional Sklyanin algebra, there is for each $z \in E$ an element $\Omega(z) \in \mathbb{P}\left(k g_{1}+k g_{2}\right)$ with the property that $\Omega(z)$ annihilates all line modules $M(p, q)$ for which $p+q=z[10, \S 6]$. Moreover, $\Omega(z)=\Omega(-z-2 \tau)$ and there are no other equalities.

PROPOSITION 4.3 [1], [14]. Let $A$ be either the 3-dimensional or 4-dimensional Sklyanin algebra. Then

$$
\operatorname{dim} \operatorname{Hom}_{A}(M(p-s \tau, q-s \tau), M(p, q)[s])=2,
$$

and, if $p-q \notin a \mathbb{Z} \tau$, any submodule of $M(p, q)[s]$ which is isomorphic to $M(p-s \tau, q-s \tau)$ is generated by a linear combination of $e_{0 s}$ and $e_{s 0}$. 
Proof. Although the result holds for all $p, q \in E$ (and is proved in this generality in [1] and [14]) we only need it in the case $p-q \notin a \mathbb{Z} \tau$ and, therefore, give a proof for that special case.

We first prove this for the 4-dimensional Sklyanin algebra. Because line modules are critical, a nonzero map between line modules is injective, so it suffices to look for suitable submodules of $M(p, q)$. It is clear that $e_{0 s}$ and $e_{s 0}$ both generate submodules of $M(p, q)$ isomorphic to $M(p-s \tau, q-s \tau)[-s]$. Therefore, to prove the lemma it suffices to prove that the only other elements generating such a submodule, are $\lambda_{1} e_{0 s}+\lambda_{2} e_{s 0}$ for $\lambda_{1}, \lambda_{2} \in k$.

Suppose this is not the case. Then there exists $0 \neq e \in M(p, q)_{s}$ of the form

$$
e=\alpha_{i} e_{i j}+\alpha_{i+1} e_{i+1, j-1}+\cdots+\alpha_{i+m} e_{i+m, j-m}
$$

where

$$
\alpha_{i} \alpha_{i+m} \neq 0 \text { and } 0<i<s-m \leqslant s,
$$

with the property that

$$
A e \cong M(p-s \tau, q-s \tau)[-s] .
$$

Let $u, v \in A_{1}$ be such that $\mathscr{V}(u, v)=l_{p-s \tau, q-s \tau}$. Thus, $u \cdot e=v \cdot e=0$. Since $u \cdot e=0$, the component of $u \cdot e_{i j}$ in $k e_{i, j+1}$ is zero, and the component of $u \cdot e_{i+m, j-m}$ in $k e_{i+m+1, j-m}$ is also zero. It follows from the previous remark that

$$
u(q+(i-j) \tau)=u(p+(j-m-i-m) \tau)=0 .
$$

The same argument applies to $v$. Since $i+j=s$, it follows that

$$
\{q+(2 i-s) \tau, p+(s-2 i-2 m) \tau\} \subset l_{p-s \tau, q-s \tau} .
$$

The hypothesis that $p-q \notin 2 \mathbb{Z} \tau$ forces

$$
q+(2 i-s) \tau=q-s \tau \text { and } p+(s-2 i-2 m) \tau=p-s \tau .
$$

Thus, $2 i \tau=2 m \tau=0$. But this is impossible since $0<i<s-m$. Hence, there is no such $e$.

Now we prove this for the 3-dimensional Sklyanin algebra, under the hypothesis that $p-q \notin 3 \mathbb{Z} \tau$. The proof proceeds as for the 4-dimensional case, except that now the line $l_{p-s \tau, q-s \tau}$ is defined by a single linear form, say $v$, satisfying

$$
v(q+(2 i-j) \tau)=v(p+(2 j-2 m-i-m) \tau)=0 .
$$

Since $i+j=s$, it follows that

$$
\{q+(3 i-s) \tau, p+(2 s-3 i-3 m) \tau\} \subset l_{p-s \tau, q-s \tau} .
$$

However, there are three points of $E$ lying on this line, the third being $-(p+q-2 s \tau)$. The hypothesis that

$$
p-q \notin 3 \mathbb{Z} \tau \text { and } 0<i<s-m
$$


forces

$$
q+(3 i-s) \tau=p+(2 s-3 i-3 m) \tau=-(p+q-2 s \tau) .
$$

But this also contradicts the hypothesis that $p-q \notin 3 \mathbb{Z} \tau$. Again we conclude that there can be no such $e$.

PROPOSITION 4.4. Let $A$ be either the 3-dimensional or 4-dimensional Sklyanin algebra.

(a) Let $M(p)$ be the point module for $A$ corresponding to $p \in E$. Then $M(p)^{(s)}$ is the point module for $k\left[u_{1}, \ldots, u_{d}\right]$ corresponding to $p^{\circ}$, the image of $p-(s-1) \tau$ in $E^{\prime}$.

(b) Let $M(p, q)$ be a line module with $p-q \notin a \mathbb{Z} \tau$. Then $\oplus_{i, j \geqslant 0} k e_{i s, j s}$ is a $k\left[u_{1}, \ldots, u_{d}\right]$-module, and as such is isomorphic to the line module corresponding to the secant line $l_{p \circ q^{\circ}}$ through $p^{\circ}$ and $q^{\circ}$, the images of $p-(s-1) \tau$ and $q-(s-1) \tau$ in $E^{\prime}$.

Proof. (a) To show that $M(p)^{(s)}$ is a cyclic $k\left[u_{1}, \ldots, u_{d}\right]$-module, it is enough to show that $M(p)_{s} \subset k\left[u_{1}, \ldots, u_{d}\right]_{s} \cdot M(p)_{0}$ and proceed by induction. If this is not the case, then $u_{i} \cdot M(p)_{0}=0$ for all $i$. But the action of $u_{i}$ on $M(p)$ is the same as the action of its image in $B$, namely $x_{i}^{s}$. Thus $x_{i}^{s} \cdot M(p)_{0}=0$, whence $x_{i}(p-(s-1) \tau) \ldots x_{i}(p)=0$. Hence, $u_{i}\left(p^{\circ}\right)=0$ for all $i$, which contradicts the fact that the $u_{i}$ span the global sections of the very ample $\mathscr{L}^{\prime}$. Hence $M(p)^{(s)}$ is cyclic.

Recall that if $b \in B_{i}$ then $b \cdot M(p)_{0}=0$ if and only if $b\left(p^{\sigma^{-(i-1)}}\right)=0$. Hence, $M(p)_{0}$ is killed by those elements of $k\left[u_{1}, \ldots, u_{d}\right]_{s}$ vanishing at $p-(s-1) \tau$, as required.

(b) Suppose that $A u_{i} e_{00}$ is nonzero. Recall that $u_{i}$ is $\left(\lambda_{\zeta} \circ \rho\right)$-normalizing for some $\zeta \in k$. If $x \in A_{1}$ then $x u_{i} e_{00}=\zeta^{-1} u_{i} x^{\rho-1} e_{00}$. Hence, $x \cdot u_{i} e_{00}=0$ if

$$
0=x^{\rho^{-1}}\left(l_{p q}\right)=x\left(l_{p-s \tau, q-s \tau}\right) .
$$

Therefore, $A u_{i} e_{00}$ is a quotient of $M(p-s \tau, q-s \tau)[-s]$. But line modules are critical, so $A u_{i} e_{00} \cong M(p-s \tau, q-s \tau)[-s]$. Therefore $u_{i} e_{00} \in k e_{0 s}+k e_{s 0}$ by (4.3). By induction (using the comments before (4.3)) $\oplus_{i, j \geqslant 0} k e_{i s, j s}$ is a $k\left[u_{1}, \ldots, u_{d}\right]$ submodule of $M(p, q)$.

To prove it is cyclic, we first show that $e_{0 s}, e_{s 0} \in k\left[u_{1}, \ldots, u_{d}\right] \cdot e_{00}$. Since $p-q \notin a \mathbb{Z} \tau, p^{\circ}$ and $q^{\circ}$ are distinct, whence there exists $u \in k\left[u_{1}, \ldots, u_{d}\right]_{s}$ such that $u\left(p^{\circ}\right)=0$ but $u\left(q^{\circ}\right) \neq 0$. Consider the point modules $M(p)$ and $M(q)$ which are quotients of $M(p, q)$. By (a) these contain copies of $M\left(p^{\circ}\right)$ and $M\left(q^{\circ}\right)$. The images of $\left\{e_{i s, 0} \mid i \geqslant 0\right\}$ and of $\left\{e_{0, j s} \mid j \geqslant 0\right\}$ are bases for $M\left(p^{\circ}\right)$ and $M\left(q^{\circ}\right)$, respectively. Since $u \cdot M\left(p^{\circ}\right)_{0}=0$ and $u \cdot M\left(q^{\circ}\right)_{0} \neq 0$ it follows that $u \cdot e_{00}$ is a nonzero multiple of $e_{0 s}$. Thus $e_{0 s} \in k\left[u_{1}, \ldots, u_{d}\right] \cdot e_{00}$ and similarly for $e_{s 0}$. By induction it follows that $\oplus k e_{i s, j s}$ is a cyclic module over $k\left[u_{1}, \ldots, u_{d}\right]$ and, hence, a line module since it has the appropriate Hilbert series. By (a) $M\left(p^{\circ}\right)$ and $M\left(q^{\circ}\right)$ are quotients of this line module, so it corresponds to $l_{p^{\circ q^{\circ}}}$. 
Remark. The aesthetically unpleasing result in (4.4a), that the point module $M(p)^{(s)}$ for $k\left[u_{1}, \ldots, u_{d}\right]$ corresponds to $p^{\prime}-(s-1) \tau^{\prime}$, rather than to $p^{\prime}$, is a consequence of the fact that our definition of $B$ (and, hence, $A$ ) is better suited to right modules than left modules. The problem occurs for any twisted homogeneous coordinate ring $B(X, \sigma, \mathscr{L})$ and left point module $M(p)$ : the Veronese submodule $M(p)^{(m)}$ is the point module for $B(X, \sigma, \mathscr{L})^{(m)} \cong B\left(X, \sigma^{m}, \mathscr{L}_{m}\right)$ corresponding to the point $p^{\sigma-m+1}$. However, if $N(p)$ is the right point module corresponding to $p$, then $N(p)^{(m)}$ corresponds to $p$ also. Thus, if in (4.4a), we had worked with the right point module $N(p)$, then $N(p)^{(s)}$ would have been the point module for $k\left[u_{1}, \ldots, u_{d}\right]$ corresponding to $p^{\prime}$. These comments also apply to (5.11a) below.

\section{TWISTING}

Let $R$ be a $\mathbb{Z}$-graded $k$-algebra and let $\theta \in \operatorname{Aut}_{k}(R)$. In the introduction we defined the twisted algebra $\left(R^{\theta}, *\right)$. We will call this the right twist of $R$ to distinguish it from the left twist $\left({ }^{\theta} R, \odot\right)$ which is defined as follows: as a graded $k$-vector space, ${ }^{\theta} R=R$, but the multiplication $\odot$ is given by

$$
x \odot y:=x^{\theta^{j}} y
$$

for $x \in{ }^{\theta} R_{i}, y \in{ }^{\theta} R_{j}$. The categories of graded left modules over $R$ and ${ }^{\theta} R$ are equivalent via the functor $M \rightarrow{ }^{\theta} M$, which is defined as follows: if $M$ is a graded left $R$-module, then ${ }^{\theta} M=M$ as a graded $k$-vector space, and the action of $x \in{ }^{\theta} R_{i}$ on $m \in{ }^{\theta} M_{j}$ is given by

$$
x \odot m:=x^{\theta^{j}} m \text {. }
$$

It is worth noting that there is an algebra isomorphism $\varphi:{ }^{\theta^{-1}} R \rightarrow R^{\theta}$ given by $\varphi(x)=\theta^{1-i}(x)$ for $x \in \theta^{\theta^{-1}} R_{i}$. Therefore we will apply these remarks to ${ }^{\theta^{-1}} B\left(\mathbb{P}^{\prime}, 1, \mathcal{O}_{\mathbb{P}}(1)\right)$.

One may associate to a $d$-linear $R$-module $M$, the $(d-1)$-dimensional linear subspace $\mathscr{V}\left(\operatorname{Ann}_{R_{1}}\left(M_{0}\right)\right)$ in $\mathbb{P}\left(R_{1}^{*}\right)$. It is an easy exercise to prove that, if $M$ is a linear $R$-module, then ${ }^{\theta} M$ is a linear ${ }^{\theta} R$-module, and the subspaces of $\mathbb{P}\left(R_{1}^{*}\right)$ associated to $M$ and ${ }^{\theta} M$ are the same.

If $I$ is a $\theta$-stable ideal of $R$, then $I$ is also a two-sided ideal of ${ }^{\theta} R$, and ${ }^{\theta} R / I \cong{ }^{\theta}(R / I)$. There is a partial converse to this, in that a two-sided ideal $I$ of ${ }^{\theta} R$ is a left ideal of $R$ because $I \supset R \odot I_{j}=\theta^{j}(R) I_{j}=R I_{j}$ for all $j$. However, we are concerned with twisting a commutative ring, in which case the following holds.

LEMMA 4.5. Let $R=k\left[R_{1}\right]$ be a commutative graded $k$-algebra, generated in degree 1. Let I be a two-sided ideal of ${ }^{\theta} R$, and let $M$ be a linear $R$-module. Then

(a) $I$ is an ideal of $R$, and

(b) $I M=0$ if and only if $I \odot^{\theta} M=0$. 
Proof. (a) The previous paragraph showed that $I$ is a left ideal of $R$.

(b) For all $j \geqslant 0$ we have $\theta^{j}(I) R_{j}=I \odot R_{j} \subset I$. Therefore

$$
I \odot{ }^{\theta} M_{j}=I \odot R_{j} \odot{ }^{\theta} M_{0}=\theta^{j}(I) R_{j} M_{0} \subset I M_{0} .
$$

Hence, if $I M=0$ then $I \odot{ }^{\theta} M=0$. Conversely, if $I \odot{ }^{\theta} M=0$, then $I \odot{ }^{\theta} M_{0}=0$, whence $I M_{0}=0$. However, $I=I R$ and $M=R M_{0}$ so $I M=0$.

THEOREM 4.6. Let $A$ be either the 3-dimensional or 4-dimensional Sklyanin algebra. Then

(a) $k\left[u_{1}, \ldots, u_{\mathrm{d}}\right] \cong B\left(\mathbb{P}^{\prime}, 1, \mathcal{O}_{\mathbb{P}},(1)\right)^{\theta} \cong B\left(\mathbb{P}^{\prime}, \mu, \mathcal{O}_{\mathbb{P}}(1)\right)$;

(b) If we define $z_{i}=u_{i}^{b}$ then $Z(A)=k\left[z_{1}, \ldots, z_{d}, g_{1}, \ldots, g_{m}\right]$.

(c) The central elements $z_{1}, \ldots, z_{d}$ are algebraically independent.

Proof. (a) The union of the secant lines $\left\{l_{p^{\prime} q^{\prime}} \mid p^{\prime}, q^{\prime} \in E^{\prime}\right\}$ is $\mathbb{P}^{\prime}$. The union of those for which $p^{\prime} \neq q^{\prime}$ (i.e. $p-q \notin a \mathbb{Z} \tau$ where $p, q \in E$ are preimages) is a dense subset of $\mathbb{P}^{\prime}$. By (4.4b) each of these lines corresponds to a line module for $k\left[u_{1}, \ldots, u_{d}\right]$. By the equivalence of categories and the fact that twisting preserves the variety associated to a linear module, a point on one of these lines corresponds to a point module for $k\left[u_{1}, \ldots, u_{d}\right]$. Hence $k\left[u_{1}, \ldots, u_{d}\right]$ has point modules corresponding to a dense subset of $\mathbb{P}^{\prime}$.

Let $I$ be the kernel of the surjective map $\gamma: B\left(\mathbb{P}^{\prime}, 1, \mathcal{O}(1)\right)^{\theta} \rightarrow k\left[u_{1}, \ldots, u_{d}\right]$ in (4.2). If $L$ is a point module for $k\left[u_{1}, \ldots, u_{d}\right]$ then $L \cong{ }^{\theta^{-1}} M$ for some point module $M$ over $B\left(\mathbb{P}^{\prime}, 1, \mathcal{O}(1)\right)$. But $I$ annihilates $L$, so by $(4.5) I$ (as an ideal of $B\left(\mathbb{P}^{\prime}, 1, \mathcal{O}(1)\right)$ ) annihilates $M$. Hence the point of $\mathbb{P}^{\prime}$ corresponding to $M$ (which is the same as the point corresponding to $L$ ) lies on the subvariety $\mathscr{V}(I)$. But there are point modules corresponding to a dense subset of $\mathbb{P}^{\prime}$, so $I=0$.

(b) The proof of (4.2) showed that $u_{i} u_{j}=\left(\zeta_{i} \zeta_{j}^{-1}\right)^{s} u_{j} u_{i}$. Since the relations for $B\left(\mathbb{P}^{\prime}, 1, \mathcal{O}(1)\right)^{\theta}$ are generated by $\left(d_{2}^{+1}\right)$ independent quadratic relations, it follows from (a) that these generate the ideal of relations for $k\left[u_{1}, \ldots, u_{d}\right]$. A simple exercise using the Diamond Lemma shows that $\left\{u_{1}^{i_{1}} \ldots u_{d}^{i_{d}} \mid\left(i_{1}, \ldots, i_{d}\right) \in \mathbb{N}^{d}\right\}$ is a basis for $k\left[u_{1}, \ldots, u_{d}\right]$. In particular, the elements $u_{1}^{b}, \ldots, u_{d}^{b}$ are linearly independent. Since the $b$ th power of a $\psi$-normalizing element is $\psi^{b}$-normalizing, each $u_{i}^{b}$ is central in $A$. Since the image of $u_{i}^{b}$ in $B$ is $x_{i}^{n}$, the result follows from (3.7).

(c) The PBW basis for $k\left[u_{1}, \ldots, u_{d}\right]$ shows that the set of all monomials in the $z_{i}$ is linearly independent, whence the $z_{i}$ are algebraically independent.

Notation. From now on we will write $z_{i}=u_{i}^{b}$. Thus

$$
Z(A)=k\left[u_{1}^{b}, \ldots, u_{d}^{b}\right]\left[g_{1}, \ldots, g_{m}\right] .
$$

Remarks. (1) Since $k\left[u_{1}, \ldots, u_{d}\right]$ is a twist of a $d$-dimensional polynomial ring it is a Nötherian domain of Gelfand-Kirillov dimension $d$, and it is a Koszul algebra of global dimension $d$. 
As remarked in the previous proof, $k\left[u_{1}, \ldots, u_{d}\right]$ has defining relations of the form $u_{i} u_{j}=\left(\zeta_{i} \zeta_{j}^{-1}\right)^{s} u_{j} u_{i}$, where $\zeta_{1}, \ldots, \zeta_{d}$ are the eigenvalues of $\rho$ on $B_{1}$. Since $\rho^{b}=1$ these are $b$ th-roots of 1 . In particular, if $b \mid s$, then $k\left[u_{1}, \ldots, u_{d}\right]$ is commutative. If $d=3$ and $3 \mid n$, these eigenvalues are the 3 distinct cube roots of 1 , so (after relabelling) the relations are $u_{i} u_{i+1}=\zeta^{s} u_{i+1} u_{i}$ for $i=1,2,3$ where $\zeta$ is a primitive cube root of 1 . If $d=4$ and $2 \mid n$ then the eigenvalues are $1,1,-1,-1$ (that each $\rho$-eigenspace is 2-dimensional comes from the discussion prior to (2.9)).

(2) Since $z_{1}, \ldots, z_{d}$ are algebraically independent it follows that the map $B\left(\mathbb{P}^{\prime \prime}, 1, \mathcal{O}_{\mathbb{P}^{\prime \prime}}(1)\right) \rightarrow k\left[z_{1}, \ldots, z_{d}\right]$ given in (4.2) (for $r=1$ ) is an isomorphism.

Next we determine the defining relations of $k\left[u_{1}, \ldots, u_{d}\right]\left[g_{1}, \ldots, g_{m}\right]$.

Notation. Let $M(p, q)$ be a line module for either the 3-dimensional or 4dimensional Sklyanin algebra having a basis $\left\{e_{i j}\right\}$ of the form described earlier. If $m=\Sigma \mu_{i j} e_{i j}$ then we define $\Pi(m):=\left\{(i, j) \mid \mu_{i j} \neq 0\right\}$ and call this the set of bi-degrees occurring in $m$.

THEOREM 4.7. Let $A$ be the 3-dimensional Sklyanin algebra. Then there is a relation in $k\left[u_{1}, u_{2}, u_{3}, g\right]$ of the form

$$
g^{s}+f_{3}\left(u_{1}, u_{2}, u_{3}\right)=0
$$

where $f_{3}\left(u_{1}, u_{2}, u_{3}\right)$ is a cubic form in $u_{1}, u_{2}, u_{3}$. In fact, if we identify $B\left(E^{\prime}, \mu, \mathscr{L}^{\prime}\right)_{3}$ with $B\left(E^{\prime}, 1, \mathscr{L}^{\prime}\right)_{3}$, as we may since $\mu^{3}=1$, then $f_{3}\left(u_{1}, u_{2}, u_{3}\right)$ is the cubic defining $E^{\prime}$ in $\mathbb{P}^{\prime}$.

Proof. Write $U=k\left[u_{1}, u_{2}, u_{3}\right]$. Since $\operatorname{deg}\left(\mathscr{L}^{\prime}\right)=3$ the same arguments as those used for $B(E, \sigma, \mathscr{L})$ in [4] show that $\beta: U \cong B\left(\mathrm{P}^{\prime}, \mu, \mathcal{O}(1)\right) \rightarrow B\left(E^{\prime}, \mu, \mathscr{L}^{\prime}\right)$ is injective in degrees $s$ and $2 s$ and that $\operatorname{ker}(\beta)$ is generated by a single element of degree $3 s$, which we will label $f_{3}\left(u_{1}, u_{2}, u_{3}\right)$.

Thus $f_{3} \in U \cap g A$ and we may write $f_{3}=g w$ for some $w \in A_{3 s-3}$. Now $f_{3} \in$ $k\left[u_{1}, u_{2}, u_{3}\right]^{(n)} \subset Z\left(A^{(3)}\right)$, whence $w \in Z\left(A^{(3)}\right)$ also. Thus

$$
g w \in g k\left[u_{1}, u_{2}, u_{3}, g\right]_{3 s-3}=U_{2 s} g^{s / 3}+U_{s} g^{2 s / 3}+k g^{s} .
$$

(If $s / 3 \notin \mathbb{Z}$ then the terms $U_{2 s} g^{s / 3}$ and $U_{s} g^{2 s / 3}$ do not occur.) Hence, there exists $f_{2}\left(u_{1}, u_{2}, u_{3}\right) \in U_{2 s}$ and $f_{1}\left(u_{1}, u_{2}, u_{3}\right) \in U_{s}$ and $c \in k$ such that

$$
f_{3}+f_{2} g^{s / 3}+f_{1} g^{2 s / 3}+c g^{s}=0
$$

in $A$. We will show that $f_{2}=f_{1}=0$ and that $c \neq 0$. If $(3, s)=1$ this is easy: since $s / 3$ and $2 s / 3$ are not integers both $f_{2}$ and $f_{1}$ are zero, and by the PBW basis for $U, c \neq 0$. Unfortunately the general case is more complicated. Hence in the rest of the proof we suppose that $3 \mid s$. This ensures that $3 \tau \neq 0$.

We will consider the action of $f_{3}+f_{2} g^{s / 3}+f_{1} g^{2 s / 3}+c g^{s}$ on the generator of a line module. To this end, let $p, q \in E$ be chosen with the property that $p-q \notin 3 \mathbb{Z} \tau, 2 p+q \notin 3 \mathbb{Z} \tau$ and $p+2 q \notin 3 \mathbb{Z} \tau$. Then the line $l_{p q}$ through $p$ and $q$ meets $E$ at 3 distinct points. Notice that given any $p \in E$ there are infinitely many choices of $q$ for which this is true (i.e. a general secant line has this property). Fix 
a basis $e_{i j}$ for $M(p, q)$ of the type described earlier. Recall that $A e_{i j}[i+j] \cong$ $M(p+(2 j-i) \tau, q+(2 i-j) \tau)$ and if $p$ and $q$ are replaced by $p+(2 j-i) \tau$ and $q+(2 i-j) \tau$ then the above hypotheses on $p$ and $q$ still hold.

We will consider the bi-degrees occurring in $f_{3} e_{00}, g^{s / 3} f_{2} e_{00}, g^{2 s / 3} f_{1} e_{00}$ and $g^{s} e_{00}$, and will exploit the fact that there must be some cancellation amongst these bi-degees since $\left(f_{3}+f_{2} g^{s / 3}+f_{1} g^{2 s / 3}+c g^{s}\right) e_{00}=0$. If $u \in U_{s}=k u_{1}+k u_{2}+k u_{3}$, then $\Pi\left(u e_{00}\right) \subset\{(s, 0),(0, s)\}$ by $(4.4 b)$. Hence,

$$
\begin{aligned}
& \Pi\left(f_{3} e_{00}\right) \subset\{(3 s, 0),(2 s, s),(s, 2 s),(0,3 s)\}, \\
& \Pi\left(f_{2} e_{00}\right) \subset\{(2 s 0),(s, s),(0,2 s)\}, \\
& \Pi\left(f_{1} e_{00}\right) \subset\{(s, 0),(0, s)\} .
\end{aligned}
$$

Now consider $g \cdot e_{00} \in k e_{30}+k e_{21}+k e_{12}+k e_{30}$. Since $g$ annihilates every point module $\Pi\left(g e_{00}\right) \subset\{(2,1),(1,2)\}$ and by induction

$$
\Pi\left(g^{l} e_{i j}\right) \subset\{(i, j)+\alpha(2,1)+\beta(1,2) \mid \alpha+\beta=l\} .
$$

More particularly, we have

SUBLEMMA: Suppose that $3 \tau \neq 0$, and that

$$
p-q \notin 3 \mathbb{Z} \tau, \quad 2 p+q \notin 3 \mathbb{Z} \tau \text { and } p+2 q \notin 2 \mathbb{Z} \tau \text {. }
$$

Then $\Pi\left(g e_{00}\right)=\{(2,1),(1,2)\}$

Proof. Suppose this is false. For example, suppose that $g e_{00} \in k e_{12}$. Recall the discussion in $[14, \S 1]$ on composition factors of modules of Gelfand-Kirillov dimension 1. Since $l_{p q} \cap E=\{p, q, r\}$ consists of 3 distinct points, the three point modules $M(p), M(q), M(r)$ are nonisomorphic irreducible objects in $\operatorname{Proj}(A)$. But these are all quotients of $M(p, q)$ which are killed by $g$ and, hence, are quotients of $N:=M(p, q) / A e_{12}$. Therefore, these are the composition factors of $N$. However, consider the composition series $N \supset A e_{01} / A e_{12} \supset A e_{02} / A e_{12} \supset 0$. The composition factors are respectively $M(p), M(p+2 \tau)[-1], M(q-2 \tau)[-2]$. Up to isomorphism in $\operatorname{Proj}(A)$ these are $M(p), M(p+3 \tau), M(q)$ from which it follows that $r=p+3 \tau$ and by collinearity of these points $2 p+q+3 \tau=0$. However this possibility was excluded, and from this contradiction the Sublemma follows.

The hypotheses on $p$ and $q$ allow us to apply the Sublemma with $e_{i j}$ in place of $e_{00}$. Therefore induction shows that $\{(i+2 l, j+l),(i+l, j+2 l)\} \subset \Pi\left(g^{l} e_{i j}\right)$.

Suppose that $(2 s, 0) \in \Pi\left(f_{2} e_{00}\right)$. Then

$$
\left(\frac{8 s}{3}, \frac{s}{3}\right) \in \Pi\left(g^{s / 3} f_{2} e_{00}\right)
$$

but a simple calculation shows that

$$
\left(\frac{8 s}{3}, \frac{s}{3}\right) \notin \Pi\left(f_{3} e_{00}\right) \cup \Pi\left(g^{2 s / 3} f_{1} e_{00}\right) \cup \Pi\left(g^{s} e_{00}\right)
$$


Hence the coefficient of $e_{2 s, 0}$ in $\left(f_{3}+g^{s / 3} f_{2}+g^{2 s / 3} f_{1}+c g^{s}\right) e_{00}$ is nonzero. It follows that $(2 s, 0) \notin \Pi\left(f_{2} e_{00}\right)$. Similar considerations apply to $(0,2 s)$, from which we conclude that $\Pi\left(f_{2} e_{00}\right) \subset\{(s, s)\}$. In particular it follows that $f_{2}$ annihilates the degree 0 component of $M(p)$. But this is true for all $p \in E$ so $f_{2} M(p)=0$ for all $p \in E$, whence the image of $f_{2}$ in $B$ is zero. But $\beta$ maps $U_{2 s}$ injectively to $B$ so $f_{2}=0$.

Suppose that $(s, 0) \in \Pi\left(f_{1} e_{00}\right)$. Then

$$
\left(\frac{7 s}{3}, \frac{2 s}{3}\right) \in \Pi\left(g^{2 s / 3} f_{1} e_{00}\right)
$$

but

$$
\left(\frac{7 s}{3}, \frac{2 s}{3}\right) \notin \Pi\left(f_{3} e_{00}\right) \cup \Pi\left(g^{s} e_{00}\right)
$$

Hence, $(s, 0) \notin \Pi\left(f_{1} e_{00}\right)$. A similar argument applies to $(0, s)$, whence $f_{1} e_{00}=0$. It follows (as for $f_{2}$ ) that $f_{1}=0$.

Hence there is a relation of the form $f_{3}+c g^{s}=0$. By the PBW basis for $U, c \neq 0$, so replacing $f_{3}$ by $c^{-1} f_{3}$ gives the result.

THEOREM 4.8. Let $A$ be the 3-dimensional Sklyanin algebra. The center of $A$ is $Z(A)=k\left[z_{1}, z_{2}, z_{3}, g\right]$, where $z_{1}, z_{2}, z_{3} \in A_{n}, g \in A_{3}$, and the ideal of relations among these generators is generated by a single relation of degree $3 n$ of the form

$$
\begin{aligned}
& f_{3}\left(z_{1}, z_{2}, z_{3}\right)+g^{n}=0 \quad \text { if }(3, n)=1, \\
& f_{3}\left(z_{1}, z_{2}, z_{3}\right)+3 z^{2} g^{s}+3 z g^{2 s}+g^{n}=0 \quad \text { if } 3 \mid n,
\end{aligned}
$$

where $f_{3}$ is a cubic defining $i\left(E^{\prime \prime}\right) \subset \mathbb{P}^{\prime \prime}$, and $z$ is a linear form on $\mathbb{P}^{\prime \prime}$ vanishing on the three images in $E^{\prime \prime}$ of the nine inflection points of $E^{\prime}$.

Proof. The Hilbert series of $Z(B)$ is that of a plane cubic but with the linear forms in degree $n$, namely

$$
H_{Z(B)}(t)=\frac{1-t^{3 n}}{\left(1-t^{n}\right)^{3}}
$$

Since $Z(A) / g Z(A)=Z(B)$ it follows that

$$
H_{Z(A)}(t)=\frac{1-t^{3 n}}{\left(1-t^{3}\right)\left(1-t^{n}\right)^{3}}
$$

Since the Hilbert series of the polynomial ring $k\left[Z_{1}, Z_{2}, Z_{3}, G\right]$ is $\left(1-t^{n}\right)^{-3}\left(1-t^{3}\right)^{-1}$ it follows that the ideal of relations is generated by a single relation of degree $3 n$.

Now we show that the relation of degree $3 n$ is of the prescribed form. If $(3, n)=1$, then this is just a restatement of (4.7) since $u_{i}=z_{i}$ in that case. So suppose that $3 \mid n$.

Let $f_{3} \in k\left[u_{1}, u_{2}, u_{3}\right]_{3 s}$ be as in (4.7). The $u_{i}$ may be labelled such that $u_{i}$ is $\left(\lambda_{\xi^{i}} \circ \rho\right)$-normalizing where $\zeta$ is a primitive cube root of 1 . Therefore, since $f_{3}$ is 
central, it must be a linear combination of $u_{1}^{3}, u_{2}^{3}, u_{3}^{3}, u_{1} u_{2} u_{3}$. The coefficient of $u_{1} u_{2} u_{3}$ is nonzero, otherwise the images of $z_{1}, z_{2}, z_{3}$ in $B$ would be linearly dependent. Hence we can rewrite the relation in (4.7) as

$$
\lambda u_{1} u_{2} u_{3}=z+g^{s}
$$

where $z \in k z_{1}+k z_{2}+k z_{3}$ and $0 \neq \lambda \in k$. Raising each side to the third power and rearranging the terms, we obtain a relation of the form

$$
z^{3}-\mu z_{1} z_{2} z_{3}+3 z^{2} g^{s}+3 z g^{2 s}+g^{n}=0 .
$$

Since $z^{3}-\mu z_{1} z_{2} z_{3}$ is in the kernel of the map $k\left[z_{1}, z_{2}, z_{3}, g\right] \rightarrow B$ and the image of this map is isomorphic to $B\left(E^{\prime \prime}, 1, \mathscr{L}^{\prime \prime}\right)$, it follows that $z^{3}-\mu z_{1} z_{2} z_{3}$ vanishes on $E^{\prime \prime}$. Hence, there is a relation of the required form with $f_{3}=z^{3}-\mu z_{1} z_{2} z_{3}$.

Concerning the description of $z$, the inflection points of $E^{\prime}$ are the points where one of the $u_{i}$ vanishes. Their images in $E^{\prime \prime}$ are therefore the points where some $z_{i}$ vanishes, and since $z^{3}-\mu z_{1} z_{2} z_{3}=0$ on $E^{\prime \prime}$, these are the points where $z$ vanishes. Finally, $z \neq 0$ since $E$ " is a smooth elliptic curve, not a 'triangle'.

Since the embedding of $E^{\prime}$ in $\mathbb{P}^{\prime}$ is obtained via the degree 4 line bundle $\mathscr{L}^{\prime}$, there is a pencil of quadrics in $\mathbb{P}^{\prime}$ containing $E^{\prime}$. These may be labelled as $Q\left(z^{\prime}\right),\left(z^{\prime} \in E^{\prime}\right)$ in such a way that $Q\left(z^{\prime}\right)$ is the union of the secant lines $\left\{l_{p^{\prime}} q^{\prime} \mid p^{\prime}+q^{\prime}= \pm z^{\prime}\right\}$.

THEOREM 4.9. Let $A$ be the 4-dimensional Sklyanin algebra. In $k\left[u_{1}, u_{2}, u_{3}, u_{4}, g_{1}, g_{2}\right]$ there are two relations of the form

$$
r_{i}:=f_{i}\left(u_{1}, u_{2}, u_{3}, u_{4}\right)+h_{i}\left(g_{1}, g_{2}\right)=0 \quad(i=1,2),
$$

where $f_{1}, f_{2}$ are linearly independent quadratic forms in $u_{1}, u_{2}, u_{3}, u_{4}$, and $h_{1}, h_{2}$ are degree $s$ forms in $g_{1}$ and $g_{2}$ having no common factor.

Proof. Write $U=k\left[u_{1}, u_{2}, u_{3}, u_{4}\right]$. Since $\operatorname{deg}\left(\mathscr{L}^{\prime}\right)=4$ the same arguments as those for $B(E, \sigma, \mathscr{L})$ show that $\beta: U \cong B\left(\mathbb{P}^{\prime}, \mu, \mathcal{O}_{\mathbb{P}}(1)\right) \rightarrow B\left(E^{\prime}, \mu, \mathscr{L}^{\prime}\right)$ is injective in degree $s$ and that $\operatorname{ker}(\beta)$ is generated by two (linearly independent) elements of degree $2 s$, which we will label $f_{i}\left(u_{1}, u_{2}, u_{3}, u_{4}\right),(i=1,2)$.

Since

$$
f_{i} \in U^{(2)} \subset Z\left(A^{(2)}\right) \text { and } f_{i} \in \operatorname{ker}(\beta)=g_{1} A+g_{2} A \text {, }
$$

it follows that $f_{i} \in Z\left(A^{(2)}\right) \cap\left(g_{1} A^{(2)}+g_{2} A^{(2)}\right)$. Hence, by (3.6c) applied to $A^{(2)}, f_{i} \in$ $g_{1} Z\left(A^{(2)}\right)+g_{2} Z\left(A^{(2)}\right)$. Since $\operatorname{deg}\left(f_{i}\right)=2 s$, it follows that

$$
f_{i} \in U_{s} k\left[g_{1}, g_{2}\right]_{s}+k\left[g_{1}, g_{2}\right]_{2 s} .
$$

If $s$ is odd then $f_{i} \in k\left[g_{1}, g_{2}\right]_{2 s}$. If $s$ is even this is not immediately clear, so we write $f_{i}=l_{i} m_{i}+h_{i}$ with $l_{i}$ a linear form in the $u_{j}, m_{i} \in k\left[g_{1}, g_{2}\right]_{s}$ and $h_{i} \in k\left[g_{1}, g_{2}\right]_{2 s}$. Therefore, from now on we suppose that $2 \mid n$ and we will show that $l_{i} m_{i}=0$.

We will consider the action of $f_{i}-l_{i} m_{i}-h_{i}$ on the generator $e_{00}$ of a line module. To this end, let $p, q \in E$ be chosen with the property that $p-q \notin 2 \mathbb{Z} \tau$. Fix a basis $e_{i j}$ for $M(p, q)$ of the type described earlier. Recall that $A e_{i j}[i+j] \cong$ 
$M(p+(j-i) \tau, q+(i-j) \tau)$ and if $p$ and $q$ are replaced by $p+(j-i) \tau$ and $q+(i-j) \tau$ then the hypotheses on $p$ and $q$ above still hold.

We will consider the bi-degrees occurring in $f_{i} \cdot e_{00}, l_{i} m_{i} \cdot e_{00}$, and $h_{i} \cdot e_{00}$. Since $\left(f_{i}-l_{i} m_{i}-h_{i}\right) \cdot e_{00}=0$, there must be some cancelling amongst these bi-degrees. If $u \in U_{s}=k u_{1}+k u_{2}+k u_{3}+k u_{4}$, then $\Pi\left(u e_{00}\right) \subset\{(s, 0),(0, s)\}$ by $(4.4 \mathrm{~b})$. Hence

$$
\Pi\left(f_{i} \cdot e_{00}\right) \subset\{(2 s, 0),(s, s),(0,2 s)\} \text { and } \Pi\left(l_{i} \cdot e_{00}\right) \subset\{(s, 0),(0, s)\} \text {. }
$$

Since $g_{1}$ and $g_{2}$ annihilate the point modules $M(p)$ for $p \in E, \Pi\left(g_{i} \cdot e_{00}\right) \subset\{(1,1)\}$, whence

$$
\Pi\left(h_{i} \cdot e_{00}\right) \subset\{(s, s)\} \text { and } \Pi\left(m_{i} \cdot e_{00}\right) \subset\left\{\left(\frac{s}{2}, \frac{s}{2}\right)\right\} .
$$

Since $f_{i} \in \operatorname{ker}(\beta), f_{i} \cdot M(p)=0$ for all $p \in E$. Thus, $\Pi\left(f_{i} \cdot e_{00}\right) \subset\{(s, s)\}$.

SUBLEMMA. Suppose that $m_{i} \neq 0$. For a dense set of lines

$$
\Pi\left(m_{i} \cdot e_{00}\right)=\left\{\left(\frac{s}{2}, \frac{s}{2}\right)\right\} .
$$

Proof. Since $m_{i} \in k\left[g_{1}, g_{2}\right]_{s}$, it is a product of $s / 2$ linear forms in $g_{1}, g_{2}$. Hence, we may write $m_{i}=\Omega\left(z_{1}\right) \ldots \Omega\left(z_{s / 2}\right)$, where $\Omega\left(z_{j}\right)$ annihilates precisely those line modules $M\left(p_{1}, q_{1}\right)$ such that $p_{1}+q_{1} \in\left\{z_{j},-2 \tau-z_{j}\right\}$, and acts faithfully on all the other line modules. In particular, each $\Omega\left(z_{j}\right)$ acts faithfully on a dense set of line modules. Hence, so does $m_{i}$, and the result follows.

Similarly, for a dense set of lines $\Pi\left(h_{i} \cdot e_{00}\right)=\{(s, s)\}$. We may also prove if $l_{i} \neq 0$ that for a dense set of lines $\Pi\left(l_{i} \cdot e_{00}\right)=\{(s, s)\}$. In summary, if $l_{i} m_{i} \neq 0$ there is a dense set of lines for which

$$
\Pi\left(f_{i} \cdot e_{00}\right) \subset\{(s, s)\}, \quad \Pi\left(l_{i} m_{i} \cdot e_{00}\right)=\left\{\left(\frac{3 s}{2}, \frac{3 s}{2}\right)\right\}, \quad \Pi\left(h_{i} \cdot e_{00}\right)=\{(s, s)\} .
$$

It follows at once that $\left(f_{i}-l_{i} m_{i}-h_{i}\right) \cdot e_{00} \neq 0$ for these lines. This is a contradiction, so we conclude that $l_{i} m_{i}=0$.

To show that $h_{1}, h_{2}$ have no common factor we need a more precise description of them. Fix one of the relations $f+h=0$ with $f \in U_{2 s}$ and $h \in k\left[g_{1}, g_{2}\right]_{2 s}$. Since $f$ is central, $U f$ is a 2-sided ideal of $U \cong B\left(\mathbb{P}^{\prime}, 1, \mathcal{O}(1)\right)^{\theta}$ and, hence, of $B\left(\mathbb{P}^{\prime}, 1, \mathcal{O}(1)\right)$ by $(4.5 \mathrm{a})$. By $(4.5 \mathrm{~b})$, the line modules (respectively, point modules) for $U / U f$ are all of the form ${ }^{\theta} M$ where $M$ is a line module (respectively, point module) for $B\left(\mathbb{P}^{\prime}, 1, \mathcal{O}(1)\right)$ which is killed by $f$ (viewed as an element of $\left.B\left(\mathbb{P}^{\prime}, 1, \mathcal{O}(1)\right)\right)$. Since twisting preserves the variety associated to a linear module, the line (respectively, point) modules for $U / U f$ correspond to the lines (respectively, points) lying on the quadric $\mathscr{V}(f) \subset \mathbb{P}^{\prime}$.

Since $f \in k\left[g_{1}, g_{2}\right]_{2 s}$ it annihilates all the $M(p)$ and, since $M(p)$ contains a copy of $M\left(p^{\circ}\right), U / U f$ has point modules corresponding to every $p^{\circ} \in E^{\prime}$. Therefore, $\mathscr{V}(f) \supset E^{\prime}$, and there exists $z^{\prime} \in E^{\prime}$ such that $\mathscr{V}(f)=Q\left(z^{\prime}\right)$. Therefore, the line modules for $U / U f$ are precisely the $M\left(p^{\prime}, q^{\prime}\right)$ for $p^{\prime}+q^{\prime}= \pm z^{\prime}$.

Let $z \in E$ be such that no two of $\{\Omega(z+j b \tau) \mid 0 \leqslant j \leqslant s-1\}$ annihilate the same line module. Let $z^{\prime}$ be the image of $z$ in $E^{\prime}$. Now choose $0 \neq f \in k f_{1}+k f_{2}$ such that 
$\mathscr{V}(f)=Q\left(z^{\prime}\right)$ and let $f+h=0$ be the corresponding relation. We will show that

$$
h=\prod_{j=0}^{s-1} \Omega(z+j b \tau) .
$$

Fix $j$ and pick $p, q$ such that

$$
p+q=z+j b \tau \text { and } p-q \notin 2 \mathbb{Z} \tau \text {. }
$$

Let $p^{\circ}, q^{\circ} \in E^{\prime}$ be the images of $p-(s-1) \tau$ and $q-(s-1) \tau$. Thus, $p^{\circ}+q^{\circ}=z^{\prime}$ since the image of $2 \tau$ in $E^{\prime}$ is zero. Therefore, $f \cdot M\left(p^{\circ}, q^{\circ}\right)=0$. But $M\left(p^{\circ}, q^{\circ}\right)$ embeds in $M(p, q)$ by $(4.4 b)$, so $f$ annihilates $M(p, q)_{0}$. Since $f$ is central $0=$ $f \cdot M(p, q)=h \cdot M(p, q)$. But $\operatorname{Ann}_{k\left[g_{1}, g_{2}\right]} M(p, q)$ is generated by $\Omega(p+q) \in k\left[g_{1}, g_{2}\right]$. To see this, first observe that this annihilator is a prime ideal since $M(p, q)$ is critical. This prime ideal is not maximal since $M(p, q)$ is not a $B$-module. Thus $h$ is divisible by $\Omega(z+j b \tau)$. Since this is true for all $j, h$ has the required property.

Choose $z_{1}, z_{2}$ such that no two of $\left\{\Omega\left(z_{i}+j b \tau\right) \mid 0 \leqslant j \leqslant s-1, i=1,2\right\}$ annihilate the same line module. The above argument applied to $z_{1}$ and $z_{2}$ gives relations $f_{i}+h_{i}=0(i=1,2)$ such that

$$
h_{i}=\prod_{j=0}^{s-1} \Omega\left(z_{i}+j b \tau\right) \text {. }
$$

The careful choice of $z_{1}$ and $z_{2}$ ensures that $h_{1}$ and $h_{2}$ have no common factor.

THEOREM 4.10. Let $A$ be the 4-dimensional Sklyanin algebra. Then $Z(A)=$ $k\left[z_{1}, z_{2}, z_{3}, z_{4}, g_{1}, g_{2}\right]$ and the ideal of relations is generated by two relations of degree $2 n$, both of which are of the form

$$
\begin{aligned}
& q_{i}\left(z_{1}, z_{2}, z_{3}, z_{4}\right)+h_{i}\left(g_{1}, g_{2}\right)=0 \quad(i=1,2) \quad \text { if }(2, n)=1, \\
& q_{i}\left(z_{1}, z_{2}, z_{3}, z_{4}\right)+l_{i}\left(z_{1}, z_{2}, z_{3}, z_{4}\right) h_{i}\left(g_{1}, g_{2}\right)+h_{i}\left(g_{1}, g_{2}\right)^{2}=0 \quad(i=1,2) \quad \text { if } 2 \mid n,
\end{aligned}
$$

where $q_{1}, q_{2}$ are quadratic forms defining $i\left(E^{\prime \prime}\right) \subset \mathbb{P}^{\prime \prime}, l_{i}$ are linear forms and $h_{i} \in$ $k\left[g_{1}, g_{2}\right]_{2 s}$.

Proof. First we show that there are two relations of degree $2 n$ of the prescribed form. If $n$ is odd then $s=n$ and $z_{i}=u_{i}$, so the theorem is just a restatement of (4.9). Suppose that $n=2 s$, so $z_{i}=u_{i}^{2}$.

We may label the $u_{i}$ such that $u_{1}$ and $u_{4}$ are $\rho$-normalizing, whereas $u_{2}$ and $u_{3}$ are $\left(\lambda_{-1} \circ \rho\right)$-normalizing. Thus

$$
Z(A) \cap k\left[u_{1}, \ldots, u_{4}\right]_{2 s}=k u_{1}^{2}+\cdots+k u_{4}^{2}+k u_{1} u_{4}+k u_{2} u_{3} .
$$

Let $r=f\left(u_{1}, \ldots, u_{4}\right)+h\left(g_{1}, g_{2}\right)$ be any linear combination of the relations $r_{i}:=f_{i}\left(u_{1}, \ldots, u_{4}\right)+h_{i}\left(g_{1}, g_{2}\right)$ occurring in (4.9). If $f \in k u_{1}^{2}+\cdots+k u_{4}^{2}$, then the images of $z_{1}, \ldots, z_{4}$ in $B$ are linearly dependent, which contradicts the fact that $\operatorname{dim} Z(B)_{n}=4$. Hence, $f \notin k u_{1}^{2}+\cdots+k u_{4}^{2}$. Therefore, we can choose $r_{1}$ and $r_{2}$ such 
that

$$
f_{1} \in u_{1} u_{4}+k u_{1}^{2}+\cdots+k u_{4}^{2} \text { and } f_{2} \in u_{2} u_{3}+k u_{1}^{2}+\cdots+k u_{4}^{2} .
$$

Consider $r_{1}$. This can be written as $u_{1} u_{4}=a_{1}-h_{1}$ where $a_{1} \in k z_{1}+\cdots+k z_{4}$. Squaring both sides of this gives a relation

$$
a_{1}^{2}-z_{1} z_{4}-2 a_{1} h_{1}+h_{1}^{2}=0
$$

of the prescribed form with $q_{1}=a_{1}^{2}-z_{1} z_{4}$. From $r_{2}$, we obtain a similar relation with $q_{2}=a_{2}^{2}-z_{2} z_{3}$ for some $a_{2} \in k z_{1}+\cdots+z_{4}$.

It remains to show that $q_{1}$ and $q_{2}$ are defining equations for $E^{\prime \prime}$. The same sort of argument as in the proof of Theorem 4.8 shows that $q_{1}$ and $q_{2}$ vanish on $E^{\prime \prime}$, so it suffices to show that $q_{1}$ and $q_{2}$ are linearly independent. If $\mu q_{1}+q_{2}=0$ for some $0 \neq \mu \in k$ then $\mu a_{1}^{2}+a_{2}^{2}=\mu z_{1} z_{4}+z_{2} z_{3}$. But this is impossible, since the left-hand side is a reducible element of the polynomial ring $k\left[z_{1}, \ldots, z_{4}\right]$ whereas the righthand side is irreducible.

Consider the relations $r_{1}$ and $r_{2}$ as elements of the polynomial ring $k\left[Z_{1}, Z_{2}, Z_{3}, Z_{4}, G_{1}, G_{2}\right]$. The Hilbert series of this polynomial ring is $\left(1-t^{n}\right)^{-4}\left(1-t^{2}\right)^{-2}$. Since $Z(B)=Z(A) /\left(g_{1}, g_{2}\right)$, where $g_{1}, g_{2}$ is a regular sequence,

$$
H_{Z(A)}(t)=\frac{\left(1+t^{n}\right)^{2}}{\left(1-t^{n}\right)^{2}\left(1-t^{2}\right)^{2}} .
$$

By comparing these two Hilbert series, one sees that if $r_{1}, r_{2}$ form a regular sequence, then they generate the ideal of relations for $Z(A)$. Thus it suffices to prove that $r_{1}$ is irreducible. Write $r_{1}=q_{1}+\left(r_{1}-q_{1}\right)$ where

$$
q_{1} \in k\left[Z_{1}, Z_{2}, Z_{3}, Z_{4}\right] \text { and } r_{1}-q_{1} \in\left(G_{1}, G_{2}\right) \text {. }
$$

If $r_{1}$ is reducible then it factors as a product of homogeneous elements, say $r_{1}=\left(x+x^{\prime}\right)\left(y+y^{\prime}\right)$ where

$$
x, y \in k\left[Z_{1}, \ldots, Z_{4}\right] \text { and } x^{\prime}, y^{\prime} \in\left(G_{1}, G_{2}\right) .
$$

Thus, $q_{1}=x y$, but $q_{1}$ is irreducible (since $E^{\prime \prime}$ is not contained in a hyperplane) so, without loss of generality, $x=1$. But $x+x^{\prime}$ is homogeneous, so $x+x^{\prime} \in k$. Therefore $r_{1}$ is irreducible, as required.

PROPOSITION 4.11. Let $A$ be a locally finite graded $k$-algebra which is bounded below, and suppose that $f_{1}, \ldots, f_{r} \in A$ is a regular sequence of homogeneous central elements of positive degree. Define $B=k\left[f_{1}, \ldots, f_{r}\right]$ and $\mathrm{m}=B f_{1}+\cdots+B f_{r}$. Then

(a) $A$ is a free $B$-module;

(b) $H_{A}(t)=H_{B}(t) \cdot H_{A / A m}(t)$.

Proof. It follows from the hypotheses that $B$ is a polynomial ring with Hilbert series $H_{B}(t)=\Pi_{1 \leqslant i \leqslant r}\left(1-t^{d_{i}}\right)^{-1}$, where $d_{i}=\operatorname{deg}\left(f_{i}\right)$, and that

$$
H_{A / \mathrm{m} A}(t)=H_{A}(t) \Pi_{1 \leqslant i \leqslant r}\left(1-t^{d_{i}}\right) .
$$


Choose a graded vector space $V \subset A$ which maps isomorphically onto $A / \mathrm{m} A$ under the quotient map $A \rightarrow A / \mathrm{m} A$. The multiplication map $A \otimes_{k} A \rightarrow A$ induces a degree zero map of graded $B$-modules $\varphi: B \otimes_{k} V \rightarrow A$, where $B \otimes V$ is given the tensor product grading. Since

$$
H_{B \otimes V}(t)=H_{B}(t) H_{A / \mathrm{mA} A}(t)=H_{A}(t)
$$

$\varphi$ is an isomorphism if and only if it is surjective. Define $X=\operatorname{coker}(\varphi)$. Applying the functor $(B / \mathrm{m}) \otimes_{B}-$ to $B \otimes_{k} V \rightarrow A \rightarrow X \rightarrow 0$ gives an exact sequence $V \rightarrow A / \mathrm{m} A \rightarrow X / \mathrm{m} X \rightarrow 0$. The definition of $V$ ensures that $X / \mathrm{m} X=0$. Hence, by the graded version of Nakayama's lemma, $X=0$.

COROLLARY 4.12. In the 4-dimensional Sklyanin algebra, let $h_{1}, h_{2}$ be as in (4.9). Then $k\left[g_{1}, g_{2}\right]$ is a free $k\left[h_{1}, h_{2}\right]$-module of rank $s^{2}$, and

$$
H_{k\left[g_{1}, g_{2}\right]}(t)=\frac{\left(1-t^{2 s}\right)^{2}}{\left(1-t^{2}\right)^{2}} H_{k\left[h_{1}, h_{2}\right]}(t) \text {. }
$$

Proof. Since $h_{1}$ and $h_{2}$ have no common factor they form a regular sequence on $k\left[g_{1}, g_{2}\right]$. Thus, $k\left[g_{1}, g_{2}\right]$ is a free $k\left[h_{1}, h_{2}\right]$-module by (4.11), and its rank is given by evaluating $\left(1-t^{2 s}\right)^{2}\left(1-t^{2}\right)^{-2}$ at $t=1$.

PROPOSITION 4.13. Let $A$ be either the 3-dimensional or 4-dimensional Sklyanin algebra. Let $z_{1}, \ldots, z_{d}$ be the central elements in Theorem 4.6. Then $A$ is a finitely generated $k\left[z_{1}, \ldots, z_{d}\right]$-module.

Proof. By Theorem 3.7, $A$ is a finite $Z(A)$-module, so it is enough to prove that $Z(A)$ is a finitely generated $k\left[z_{1}, \ldots, z_{d}\right]$-module. This is clear in the 3-dimensional case because (4.8) shows that $Z(A)$ is integral over $k\left[z_{1}, z_{2}, z_{3}\right]$. In the 4-dimensional case, $g_{1}$ and $g_{2}$ are integral over $k\left[h_{1}, h_{2}\right]$-module by (4.12), and $h_{1}$ and $h_{2}$ are integral over $k\left[z_{1}, z_{2}, z_{3}, z_{4}\right]$ by $(4.10)$, whence $Z(A)=k\left[z_{1}, z_{2}, z_{3}, z_{4}, g_{1}, g_{2}\right]$ is finitely generated over $k\left[z_{1}, z_{2}, z_{3}, z_{4}\right]$.

\section{The Center of $\mathscr{A}$}

Let $S=\operatorname{Proj}(Z(A))$ and let $\mathscr{A}$ be the sheaf of $\mathcal{O}_{S}$-algebras such that $\mathscr{A}\left(S_{(f)}\right)=$ $A\left[f^{-1}\right]_{0}$ for each non-zero homogeneous $f \in Z(A)$. Let $\mathscr{Z}$ denote the center of $\mathscr{A}$. Our goal is to understand $\mathscr{Z}$.

LEMMA 5.1. Let $A$ be a $\mathbb{Z}$-graded algebra over a field $k$. Suppose $Z(A)=$ $k\left[z_{1}, \ldots, z_{m}\right]$, with each $z_{i}$ being a homogeneous nonzero-divisor, of degree $n_{i}$, say. Let $b:=\operatorname{gcd}\left(n_{1}, \ldots, n_{m}\right)$. Suppose $A$ is finite over $Z(A)$. Let $S=\operatorname{Proj}(Z(A))$ and let $\mathscr{A}$ be the sheaf of $\mathcal{O}_{S}$-algebras such that $\mathscr{A}\left(S_{\left(z_{i}\right)}\right)=A\left[z_{i}^{-1}\right]_{0}$ for each $i$. Let $\mathscr{Z}$ denote the center of $\mathscr{A}$. Then

$\operatorname{Spec} \mathscr{Z} \cong \operatorname{Proj}\left(Z\left(A^{(b)}\right)\right)$. 
Proof. Fix an $i$ and write $z=z_{i}$ and $n=n_{i}$. Since $S$ is covered by the open affine sets $S_{\left(z_{i}\right)}$, we must show that

$$
Z\left(A\left[z^{-1}\right]_{0}\right)=Z\left(A^{(b)}\right)\left[z^{-1}\right]_{0} .
$$

Since the degree of $z^{-1}$ is divisible by $b$, we have $A\left[z^{-1}\right]_{0}=A^{(b)}\left[z^{-1}\right]_{0}$. Hence, replacing $A$ by $A^{(b)}$ and dividing all degrees by $b$, we can assume $b=1$ and $A=A^{(b)}$. Similarly,

$$
A\left[z^{-1}\right]_{0}=A^{(n)}\left[z^{-1}\right]_{0} \text { and } Z(A)\left[z^{-1}\right]_{0}=\left(Z(A)^{(n)}\right)\left[z^{-1}\right]_{0} .
$$

So we must show that, if $\operatorname{gcd}\left(n_{j}\right)=1$, then

$$
Z\left(\left(A\left[z^{-1}\right]\right)_{0}\right)=\left(Z\left(A\left[z^{-1}\right]\right)\right)_{0} .
$$

This follows from the fact that an element $y \in A\left[z^{-1}\right]$ which commutes with elements of degree 0 is in the center of that ring. To show that such a $y$ commutes with an element $x$ of degree $d$, say, it is enough to exhibit a central nonzero-divisor $u$ of degree $-d$, for then $y$ will commute with $x u$ and, hence, with $x$. To construct $u$, write

$$
-d=a_{1} n_{1}+\cdots+a_{i} n_{i}+\cdots+a_{m} n_{m},
$$

with $a_{j} \geqslant 0$ for $j \neq i$ and put $u=z_{1}^{a_{1}} \ldots z_{i}^{a_{i}} \ldots z_{m}^{a_{m}}$.

THEOREM 5.2. Let $A$ be either the 3-dimensional or 4-dimensional Sklyanin algebra. Then

$$
\begin{aligned}
\operatorname{Spec} \mathscr{Z} & \cong \operatorname{Proj}\left(Z\left(A^{(b)}\right)\right) \\
& =\operatorname{Proj}\left(k\left[u_{1}, u_{2}, u_{3}, u_{4}\right]^{(n)}\left[g_{1}, g_{2}\right]\right) .
\end{aligned}
$$

Proof. By Theorem 3.7,

$$
Z\left(A^{(b)}\right)=k\left[u_{1}, u_{2}, u_{3}, u_{4}\right]^{(n)}\left[g_{1}, g_{2}\right] .
$$

Again by Theorem $3.7, A$ is finite over

$$
Z(A)=k\left[z_{1}, \ldots, z_{d}, g_{1}, \ldots, g_{m}\right]
$$

where each $g_{i}$ is homogeneous of degree $a$, and each $z_{i}$ is homogeneous of degree $n$. The result follows from (5.1).

Notice that

$$
\operatorname{Proj}\left(k\left[u_{1}, \ldots, u_{d}\right]^{(n)}\right)=\operatorname{Proj}\left(k\left[u_{1}, \ldots, u_{d}\right]\right)=\operatorname{Proj} B\left(\mathbb{P}^{\prime}, 1, \mathcal{O}_{\mathbb{B}},(1)\right)=\mathbb{P}^{\prime} .
$$

Therefore, the inclusion $k\left[u_{1}, \ldots, u_{d}\right]^{(n)} \subset Z\left(A^{(b)}\right)$ induces a morphism Spec $\mathscr{Z} \rightarrow \mathbb{P}^{\prime}$. For $d=3$, we show that this is an isomorphism and thereby give a new proof of Artin's result [1] that $\operatorname{Spec} \mathscr{Z} \cong \mathbb{P}^{2}$.

THEOREM 5.3. If $A$ is the 3-dimensional Sklyanin algebra then the natural map Spec $\mathscr{Z} \rightarrow \mathbb{P}^{\prime}$ is an isomorphism, so Spec $\mathscr{Z} \cong \mathbb{P}^{2}$. 
Proof. For a graded commutative algebra $C, \operatorname{Proj}(C)=\operatorname{Proj}\left(C^{(d)}\right)$ so we may replace a commutative ring by a Veronese subalgebra when computing its Proj. Hence, by (5.2) $\operatorname{Spec} \mathscr{Z} \cong \operatorname{Proj}\left(Z\left(A^{(b)}\right)^{(3 s)}\right)$. However, $Z\left(A^{(b)}\right)^{(3 s)}=k\left[u_{1}, u_{2}, u_{3}\right]^{(3 s)}$ by (3.7) and (4.7). But

$$
k\left[u_{1}, u_{2}, u_{3}\right] \cong B\left(\mathbb{P}^{\prime}, 1, \mathcal{O}(1)\right)^{\theta} \text { and } \theta^{3}=1
$$

so $k\left[u_{1}, u_{2}, u_{3}\right]^{(3 s)} \cong B\left(\mathbb{P}^{\prime}, 1, \mathcal{O}(1)\right)^{(3)}$, from which the result follows.

\section{Spec $\mathscr{Z}$ FOR THE 4-DIMENSIONAL SKLYANIN ALGEBRA}

For the rest of this section $A$ denotes the 4-dimensional Sklyanin algebra. Let $f_{i}+h_{i},(i=1,2)$ be the defining relations in $k\left[u_{1}, u_{2}, u_{3}, u_{4}, g_{1}, g_{2}\right]$ described in (4.9); they are of degree $2 s$.

\section{LEMMA 5.4.}

(a) $Z\left(A^{(b)}\right)=k\left[u_{1}, u_{2}, u_{3}, u_{4}\right]^{(n)} \otimes_{k\left[h_{1}, h_{2}\right]} k\left[g_{1}, g_{2}\right]$,

(b) $Z\left(A^{(b)}\right)\left[f_{i}^{-1}\right]_{0}=k\left[u_{1}, u_{2}, u_{3}, u_{4}\right]^{(n)}\left[f_{i}^{-1}\right]_{0} \otimes_{k\left[f_{1}, f_{2}, f_{i}^{-1}\right]_{0}} k\left[g_{1}, g_{2}, h_{i}^{-1}\right]_{0}$.

Proof. (a) Write

$$
R=k\left[h_{1}, h_{2}\right], \quad U=k\left[u_{1}, u_{2}, u_{3}, u_{4}\right] \text { and } T=k\left[g_{1}, g_{2}\right] .
$$

Since $f_{1}, f_{2} \in U^{(n)}$ and they form a regular sequence on $U$, they also form a regular sequence on $U^{(n)}$. Thus, by (4.11), $U^{(n)}$ is a free $R$-module. Since multiplication gives a surjective map $U^{(n)} \otimes_{R} T \rightarrow Z\left(A^{(b)}\right)$, it suffices to show that $U^{(n)} \otimes_{R} T$ and $Z\left(A^{(b)}\right)$ have the same Hilbert series. Since both $U^{(n)}$ and $T$ are free $R$-modules,

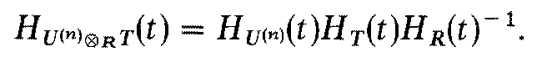

However, $Z\left(A^{(b)}\right) /\left(g_{1}, g_{2}\right)=U^{(n)} /\left(f_{1}, f_{2}\right)$ so (using the fact that $g_{1}, g_{2}$ is a regular sequence)

$$
H_{Z\left(A^{(b)}\right)}(t) H_{T}(t)^{-1}=H_{U^{(n)}}(t) H_{R}(t)^{-1} .
$$

The required equality of Hilbert series follows.

(b) Fix $i$ and write $f=f_{i}$. By (a) we have

$$
Z\left(A^{(b)}\right)\left[f^{-1}\right]=\left(U^{(n)} \otimes_{R} T\right)\left[f^{-1}\right]=U^{(n)}\left[f^{-1}\right] \otimes_{R\left[f^{-1}\right]} T\left[f^{-1}\right] .
$$

The right-hand side of this is of the form $M \otimes_{S} N$, where $S=S_{0}\left[f, f^{-1}\right]$. If $n$ is even, then

$$
M=U^{(n)}\left[f^{-1}\right]=U^{(2 s)}\left[f^{-1}\right]=M_{0}\left[f, f^{-1}\right]=M_{0} \otimes_{S_{0}} S .
$$

If $n$ is odd, then

$$
M=\left(M_{0} \oplus M_{n}\right)\left[f, f^{-1}\right]=\left(M_{0} \oplus M_{n}\right) \otimes_{S_{0}} S .
$$


Hence, if $n$ is even $M \otimes_{\mathrm{S}} N=M_{0} \otimes_{\mathrm{S}_{0}} N$, and if $n$ is odd $M \otimes_{S} N=\left(M_{0} \oplus M_{n}\right) \otimes_{\mathrm{S}_{0}} N$. However, $N=\oplus N_{j}$ is a direct sum of $S_{0}$-submodules, so

$$
\left(M \otimes_{S} N\right)_{0}=M_{0} \otimes_{S_{0}} N_{0} \quad \text { if } n \text { is even }
$$

and

$$
\left(M \otimes_{S} N\right)_{0}=\left(M_{0} \otimes_{S_{0}} N_{0}\right) \oplus\left(M_{n} \otimes_{S_{0}} N_{-n}\right) \text { if } n \text { is odd }
$$

However, $N_{-n}=0$ if $n$ is odd, so in both cases $\left(M \otimes_{S} N\right)_{0}=M_{0} \otimes_{S_{0}} N_{0}$. This proves (b).

The tensor product decomposition in (5.4b) leads to a fiber product decomposition of a Zariski dense open subset of $\operatorname{Spec} \mathscr{Z}$. To describe this, we first define the following varieties

$$
\begin{aligned}
& S=\operatorname{Proj}\left(k\left[g_{1}, g_{2}\right]\right), \\
& S^{\prime}=\operatorname{Proj}\left(k\left[f_{1}, f_{2}\right]\right)=\operatorname{Proj}\left(k\left[h_{1}, h_{2}\right]\right) .
\end{aligned}
$$

Both $\mathbb{P}^{\prime}$ and Spec $\mathscr{Z}$ contain copies of $E^{\prime}$, namely

$$
\mathscr{N}\left(f_{1}, f_{2}\right)=\operatorname{Proj}\left(k\left[u_{1}, u_{2}, u_{3}, u_{4}\right] /\left(f_{1}, f_{2}\right)\right) \cong E^{\prime}
$$

and

$$
\mathscr{r}\left(g_{1}, g_{2}\right)=\operatorname{Proj}\left(k\left[u_{1}, u_{2}, u_{3}, u_{4}\right]^{(m)}\left[g_{1}, g_{2}\right] /\left(g_{1}, g_{2}\right)\right) \cong E^{\prime} .
$$

The next proposition describes the relation between these varieties.

\section{PROPOSITION 5.5 .}

(a) There is a commutative diagram as follows (with the two vertical maps defined on the complement of the copies of $E^{\prime}$ in $\operatorname{Spec} \mathscr{Z}$ and $\left.\mathbb{P}^{\prime}\right)$ :

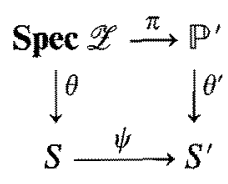

(b) The morphism $\pi$ maps $E^{\prime} \subset \operatorname{Spec} \mathscr{Z}$ isomorphically onto $E^{\prime} \subset \mathbb{P}^{\prime}$.

(c) $($ Spec $\mathscr{Z}) \backslash E^{\prime} \cong\left(\mathbb{P}^{\prime} \backslash E^{\prime}\right) \times_{S^{\prime}} S$.

Proof. (a) The maps in the diagram all arise from the obvious inclusions of the corresponding subalgebras of $Z\left(A^{(b)}\right)$. For example $\pi$ is induced by the inclusion $k\left[u_{1}, u_{2}, u_{3}, u_{4}\right]^{(n)} \subset Z\left(A^{(b)}\right)$.

(b) This is clear.

(c) Since $\mathbb{P}^{\prime} \backslash E^{\prime}$ is covered by the two open affine sets $f_{i} \neq 0$, (Spec $\left.\mathscr{Z}\right) \backslash E^{\prime}$ is covered by the two open affine sets $\operatorname{Spec}\left(Z\left(A^{(b)}\right)\left[f_{i}^{-1}\right]_{0}\right)$. By $(5.4 b)$ this ring decomposes as a suitable tensor product, thus giving the result. 
To describe Spec $\mathscr{Z}$ in more detail we need to understand the map $\psi: S \rightarrow S^{\prime}$, which requires a precise description of the relations $f_{i}+h_{i}$ occurring in (4.9). An explicit description of $\psi$ is given in Lemma 5.8.

LEMMA 5.6. The map $E \rightarrow \mathbb{P}\left(k g_{1}+k g_{2}\right)$ defined by $z \rightarrow \Omega(z)$ is a morphism.

Proof. Fix $p \in E$. Write $R_{A} \subset R_{B} \subset A_{1} \otimes A_{1}$ for the spaces of relations for $A$ and $B$, respectively. The map $z \rightarrow \Omega(z)$ may be described as a composition of simpler maps as follows. First take $z \rightarrow z-p$. Secondly, let $k u+k v \subset A_{1}$ be the space of linear forms vanishing on the secant line $l_{p, z-p}$. Thus, $k u+k v$ is an element of the Grassmanian $G\left(2, A_{1}\right)$. We may also describe $k u+k v$ as the orthogonal to the secant line $l_{p, z-p}$, which makes it clear that the map $z-p \rightarrow k u+k v$ is a morphism $E \rightarrow G\left(2, A_{1}\right)$. By $[10, \S 4] \operatorname{dim}\left(\left(A_{i} \otimes(k u+k v)\right) \cap R_{B}\right)=2$, so next we send $k u+k v$ to this 2-dimensional subspace of $R_{B}$. Again by $[10, \S 4]$, this subspace of $R_{B}$ has a 1-dimensional image in $R_{B} / R_{A}$. This may be considered as a point of $\mathbb{P}\left(k g_{1}+k g_{2}\right)$, and as such it annihilates $A / A u+A v \cong M(p, z-p)$. Hence, it is $\Omega(z)$. Thus, we have written $z \rightarrow \Omega(z)$ as a composition of maps

$$
E \rightarrow E \rightarrow G\left(2, A_{1}\right) \rightarrow G\left(2, R_{B}\right) \rightarrow \mathbb{P}\left(R_{A} / R_{B}\right)=\mathbb{P}\left(k g_{1}+k g_{2}\right),
$$

where the maps $G\left(2, A_{1}\right) \rightarrow G\left(2, R_{B}\right) \rightarrow \mathbb{P}\left(R_{A} / R_{B}\right)$ are only defined on the image of the previous map. It is clear that each individual map is a morphism, hence so is their composition.

LEMMA 5.7. If $z^{\prime} \in E^{\prime}$ let $Q\left(z^{\prime}\right) \subset \mathbb{P}^{\prime}$ denote the quadric containing $E^{\prime}$ which is the union of the secant lines $l_{p^{\prime}, z^{\prime}-p^{\prime}}$ for $p^{\prime} \in E^{\prime}$. Let $r_{1}$ and $r_{2}$ be a basis for the defining relations of $k\left[u_{1}, u_{2}, u_{3}, u_{4}, g_{1}, g_{2}\right]$. There is a morphism $r: E^{\prime} \rightarrow \mathbb{P}\left(k r_{1}+k r_{2}\right)$ such that $r\left(p^{\prime}\right)=f_{p^{\prime}}+h_{p^{\prime}}$, where $f_{p^{\prime}} \in k\left[u_{1}, u_{2}, u_{3}, u_{4}\right]_{2 s}$ vanishes on $Q\left(p^{\prime}\right)$, and $h_{p^{\prime}} \in k\left[g_{1}, g_{2}\right]_{2 s}$ is a nonzero scalar multiple of

$$
\prod_{p=E \text { is a preimage of } p^{\prime}} \Omega(p)=\prod_{i=0}^{s-1} \Omega(p+i b \tau)
$$

The morphism $r$ is of degree 2 and $r\left(p^{\prime}\right)=r\left(q^{\prime}\right)$ if and only if $p^{\prime}= \pm q^{\prime}$.

Proof. It is already clear from the proof of (4.9) that there is a map $r: E^{\prime} \rightarrow \mathbb{P}\left(k r_{1}+k r_{2}\right)$ such that $r\left(p^{\prime}\right)=f_{p^{\prime}}+h_{p^{\prime}}$, where $f_{p^{\prime}} \in k\left[u_{1}, \ldots, u_{4}\right]_{2 s}$ vanishes on $Q\left(p^{\prime}\right)$ and $h_{p^{\prime}}$ is some element in $k\left[g_{1}, g_{2}\right]_{2 s}$. Since the map $p^{\prime} \rightarrow k f_{p^{\prime}} \in$ $\mathbb{P}\left(k f_{1}+k f_{2}\right)$ is a morphism, it follows that $r$ is a morphism. Furthermore, for a dense set of $p^{\prime},(4.9)$ shows that $h_{p^{\prime}}$ is a scalar multiple of $\Omega(p) \Omega(p+b \tau) \ldots \Omega(p+(s-1) b \tau)$, where $p \in E$ is a preimage of $p^{\prime}$. Since the map $p \rightarrow \Omega(p)$ is a morphism it follows that $h_{p^{\prime}}$ is a scalar multiple of $\Omega(p) \Omega(p+b \tau) \ldots \Omega(p+(s-1) b \tau)$ for all $p^{\prime}$. 
LEMMA 5.8.

(a) There is a commutative diagram

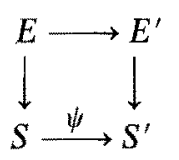

where $\psi$ is the map in (5.5a), $E \rightarrow E^{\prime}$ is the natural isogeny, $E^{\prime} \rightarrow S^{\prime}$ is the quotient map for the $\mathbb{Z}_{2}$ action $p^{\prime} \rightarrow-p^{\prime}$ and $E \rightarrow S$ is the quotient map for the $\mathbb{Z}_{2}$-action $p \rightarrow-(p+2 \tau)$ on $E$.

(b) Denote the image of $p \in E$ in $S$ by $[p]$, and the image of $p^{\prime} \in E^{\prime}$ in $S^{\prime}$ by $\left[p^{\prime}\right]$. Then

$$
[p]=\operatorname{Proj}\left(k\left[g_{1}, g_{2}\right] /(\Omega(p))\right) \text { and }\left[p^{\prime}\right]=\psi([p])=\operatorname{Proj}\left(k\left[h_{1}, h_{2}\right] /\left(h_{p^{\prime}}\right)\right) .
$$

(c) The map $\psi$ is of degree $s$, and its ramification locus is the $2 s-2$ points in

$$
\left\{[\omega+i \tau] \in S \mid \omega \in E_{2}, 0 \leqslant i \leqslant s-2\right\}
$$

which is the image of $\left(E_{2}+\mathbb{Z} \tau\right) \backslash\left(E_{2}-\tau\right)$. The ramification index is 2 at each point.

Proof. The projective lines

$$
\mathbb{P}\left(k g_{1}+k g_{2}\right) \text { and } S=\operatorname{Proj}\left(k\left[g_{1}, g_{2}\right]\right)
$$

are dual to one another. The composition $E \stackrel{\Omega}{\longrightarrow} \mathbb{P}\left(k g_{1}+k g_{2}\right) \rightarrow S$, where the second map is the isomorphism sending a line to its orthogonal, is a morphism by (5.6). Its fibers are the $\mathbb{Z}_{2}$-orbits, so it is the quotient morphism. By (5.7) the fibers of the composition

$$
E^{\prime} \rightarrow \mathbb{P}\left(k h_{1}+k h_{2}\right) \rightarrow S^{\prime}=\operatorname{Proj}\left(k\left[h_{1}, h_{2}\right]\right),
$$

defined by $p^{\prime} \rightarrow h_{p^{\prime}} \rightarrow \mathscr{V}\left(h_{p^{\prime}}\right)$, are the sets $\left\{p^{\prime},-p^{\prime}\right\}$, so this is the quotient map for the $\mathbb{Z}_{2}$-action. Since $\psi$ is induced by the inclusion $k\left[h_{1}, h_{2}\right] \subset k\left[g_{1}, g_{2}\right]$, the commutativity of the diagram follows from the fact that $\Omega(p)$ divides $h_{p^{\prime}}$. This proves (a) and (b).

The map $S \rightarrow S^{\prime}$ is of degree $s$ because both vertical maps are of degree 2 and the isogeny is of degree $s$. The map $E^{\prime} \rightarrow S^{\prime}$ is ramified precisely at the 4 points of $\left(E^{\prime}\right)_{2}$, the 2-torsion subgroup of $E^{\prime}$. Since $E \rightarrow E^{\prime}$ is étale of degree $s$, the composition $E \rightarrow E^{\prime} \rightarrow S^{\prime}$ is ramified, with ramification indices 2 , at the $4 s$ points lying over $\left(E^{\prime}\right)_{2}$. This set is $E_{2}+\mathbb{Z} \tau$. Since $E \rightarrow S$ is ramified precisely at the 4 points of $E_{2}-\tau$, it follows that the ramification locus of $S \rightarrow S^{\prime}$ is the image in $S$ of $\left(E_{2}+\mathbb{Z} \tau\right) \backslash\left(E_{2}-\tau\right)$. Since there are $4 s$ points in $E_{2}+\mathbb{Z} \tau$, this image consists of $\frac{1}{2}(4 s-4)=2 s-2$ points. 
Let $p \in E$ and write $p^{\prime}$ for its image in $E^{\prime}$. Denote by $W(p)$ the closure of the fiber $\theta^{-1}([p])$, and by $Q\left(p^{\prime}\right)$ the closure of the fiber $\theta^{\prime-1}(\psi([p]))$. Thus $\pi: \operatorname{Spec} \mathscr{Z} \rightarrow \mathbb{P}^{\prime}$ maps $W(p)$ isomorphically onto $Q\left(p^{\prime}\right)$. Since

$$
Q\left(p^{\prime}\right)=\cup\left\{l_{q^{\prime} r^{\prime}} \mid q^{\prime}, r^{\prime} \in E^{\prime}, q^{\prime}+r^{\prime}=p^{\prime}\right\}
$$

is a quadric in $\mathbb{P}^{\prime}$ containing $E^{\prime}$ we will also refer to $W(p)$ as a quadric. Since $Q\left(p^{\prime}\right)$ is singular exactly when $2 p^{\prime}=0, W(p)$ is singular exactly when $p \in E_{2}+\mathbb{Z} \tau$. By (5.8c) the image of $\left(E_{2}+\mathbb{Z} \tau\right) \backslash\left(E_{2}-\tau\right)$ in $S$ consists of $2(s-1)$ points. Since the ramification locus of $E \rightarrow S$ is $E_{2}-\tau$, the image of $E_{2}+\mathbb{Z} \tau$ in $S$ consists of $2(s-1)+4=2 s+2$ points. Thus, exactly $2 s+2$ of the quadrics $W(p)$ are singular.

\section{THEOREM 5.9.}

(a) The map $\pi$ :Spec $\mathscr{Z} \rightarrow \mathbb{P}^{\prime}$ is a flat morphism of degree $s$.

(b) The ramification locus of $\pi$ is the union of the $2 s-2$ singular quadrics

$$
\bigcup_{\omega \in E_{2}, 0 \leqslant i \leqslant s-2} W(\omega+i \tau) \text {. }
$$

Proof. (a) We must show for each $y \in \mathbf{S p e c} \mathscr{Z}$ that the scheme theoretic fiber $\pi^{-1} \pi(y)$ is the Spec of an s-dimensional $k$-algebra. The inclusion $\{\pi(y)\} \subset \mathbb{P}^{\prime}$ corresponds to a surjective map of graded algebras $k\left[u_{1}, u_{2}, u_{3}, u_{4}\right]^{(n)} \rightarrow k[z]$, where $\operatorname{deg}(z)=n$, i.e. $\{\pi(y)\}=\operatorname{Proj}(k[z])$. Hence $\pi^{-1} \pi(y)=\operatorname{Proj}\left(k\left[z, g_{1}, g_{2}\right]\right)$, where the defining relations for $k\left[z, g_{1}, g_{2}\right]$ are obtained by evaluating the relations for $Z\left(A^{(b)}\right)$ at $y$. Set $w=z$ if $n$ is even, and $w=z^{2}$ if $n$ is odd. Thus $\operatorname{deg}(w)=2 s$ and $k\left[z, g_{1}, g_{2}\right]^{(2 s)}=k\left[w, g_{1}, g_{2}\right]^{(2 s)}$

By (4.9) $k\left[w, g_{1}, g_{2}\right]$ is the quotient of the polynomial ring $C=k\left[W, G_{1}, G_{2}\right]$ by the ideal $\left(r_{1}, r_{2}\right)$ where $r_{i}:=\alpha_{i} W+h_{i}\left(G_{1}, G_{2}\right),(i=1,2)$ for some $\alpha_{1}, \alpha_{2} \in k$. Since $h_{1}$ and $h_{2}$ are without common factor in $k\left[G_{1}, G_{2}\right]$, the relations $r_{1}$ and $r_{2}$ are without common factor in $k\left[W, G_{1}, G_{2}\right]$. Hence, $r_{1}$ and $r_{2}$ form a regular sequence in $C$, and it follows that the Hilbert series of $k\left[w, g_{1}, g_{2}\right]$ is

$$
\left(1-t^{2 s}\right)^{2} H_{C}(t)=\left(1+t^{2}+\cdots+t^{2(s-1)}\right)\left(1-t^{2}\right)^{-1},
$$

i.e. the dimensions are $1,0,2,0,3,0, \ldots, s-1,0, s, 0, s, 0, \ldots$ so

$$
\operatorname{dim}\left(k\left[w, g_{1}, g_{2}\right]\left[w^{-1}\right]_{0}\right)=s
$$

as required.

(b) By (5.5c) the map $\pi$ :(Spec $\mathscr{Z}) \backslash E^{\prime} \rightarrow \mathbb{P}^{\prime} \backslash E^{\prime}$ is obtained from $\psi: S \rightarrow S^{\prime}$ by base extension. Standard results imply that $\pi$ is ramified at $y$ if and only if $\psi$ is ramified at $\theta(y)$. Hence, by $(5.8), \pi$ is ramified at $y \in(\operatorname{Spec} \mathscr{Z}) \backslash E^{\prime}$ if and only if $y \in W(\omega+i \tau)$ for some $\omega \in E_{2}$ and some $0 \leqslant i \leqslant s-2$. Now each $W(p)$ contains $E^{\prime}$, and since the ramification locus is closed, the result follows.

PROPOSITION 5.10. Spec $\mathscr{Z}$ is a normal variety.

Proof. The following short proof was pointed out to us by M. Artin. By [15] $A$ is a maximal order in $\operatorname{Fract}(A)$. Two easy exercises show that if $z$ is a central 
homogeneous element then $A\left[z^{-1}\right]$ and $A\left[z^{-1}\right]_{0}$ are maximal orders. Hence, the center of $A\left[z^{-1}\right]_{0}$ is integrally closed. Therefore Spec $\mathscr{Z}$ is normal, being a union of open affine sets, each of which is normal.

The proof of (5.10) shows that $\mathscr{A}$ is a sheaf of maximal orders over Spec $\mathscr{Z}$. The next result describes the points of $\operatorname{Spec} \mathscr{Z}$ where $\mathscr{A}$ is Azumaya.

First we need to know the fat points of $A$. A fat point of $A$ is an isomorphism class of a 1-critical graded $A$-module in the category $\operatorname{Proj}(A)$. More details may be found in [1], but we warn the reader that, in contrast to [1], we do not insist that a fat point be of multiplicity $>1$. Thus, each point module gives a fat point. Each fat point is represented by a graded $A$-module which is generated in degree zero and has constant Hilbert series. Such a representative is unique up to isomorphism of graded $A$-modules, and is called a fat point module (see [1], [5], [14]). By [5, Proposition 7.5], if $z$ is a nonzero homogeneous central element of $A$, there is a functorial bijection between the finite-dimensional simple $A\left[z^{-1}\right]_{0}$-modules and the fat points for $A$ which have no $z$-torsion. Under this bijection a fat point module $F$ corresponds to $F\left[z^{-1}\right]_{0}$. Moreover, the multiplicity of the fat point equals the dimension of the corresponding simple module.

It is proved in [14] that $A\left[z^{-1}\right]_{0}$ satisfies a polynomial identity of degree $2 s$. Therefore, by the Artin-Procesi Theorem, $A\left[z^{-1}\right]_{0}$ is Azumaya of rank $s^{2}$ if and only if every simple $A\left[z^{-1}\right]_{0}$-module is of dimension $s$. Since the annihilator of a fat point module is prime, a fat point module is supported at a unique point of Spec $\mathscr{Z}$. Strictly speaking, we should speak of the support of the $\mathscr{A}$-module $\mathscr{F}$ associated to $F$, which is defined by $\mathscr{F}\left(S_{(z)}\right)=F\left[z^{-1}\right]_{0}$. Hence, $y \in \operatorname{Spec} \mathscr{Z}$ is a non-Azumaya point for $\mathscr{A}$ if and only $y$ is the support of a fat point module of multiplicity $<s$.

The fat points for $A$ have been classified in [14]: there is a 3-parameter family of fat points of multiplicity $s$, each $p \in E$ gives a fat point of multiplicity 1 , namely the class of the point module $M(p)$, and for each $\omega \in E_{2}$ and each $j=0,1, \ldots, s-2$ there is a fat point module labelled $F(\omega+j \tau)$ which is of multiplicity $j+1$. These are all the fat points. Hence, if $s>1$, the non-Azumaya points of Spec $\mathscr{Z}$ are the points which support either a point module $M(p)$ or one of the $F(\omega+j \tau)$. The modules $F(\omega+j \tau)$ are defined in [14], but the only property of them which we will use is that there is a nonzero degree zero $A$-module map $M(p, q) \rightarrow F(\omega+j \tau)$ whenever $p, q \in E$ are such that $p+q=\omega+j \tau$.

If $s=1$ it follows from the above discussion that $\mathscr{A}$ is Azumaya at all points of Spec $\mathscr{Z}$, and from (5.9) that $\pi:$ Spec $\mathscr{Z} \rightarrow \mathbb{P}^{\prime}$ is an isomorphism. Therefore, for the remainder of the paper, we assume that $s>1$, or equivalently that $n \neq 1,2$.

\section{THEOREM 5.11 .}

(a) Let $p \in E$. Then the support of the point module $M(p)$ is $p^{\prime}-(s-1) \tau^{\prime} \in$ $E^{\prime} \subset \operatorname{Spec} \mathscr{Z}$.

(b) Let $\omega \in E_{2}$ and $0 \leqslant j \leqslant s-2$. Then the support of the fat point module $F(\omega+j \tau)$ is the singular point of $W(\omega+j \tau)$. 
(c) The non-Azumaya points of $\mathscr{A}$ in Spec $\mathscr{Z}$ are the points of $E^{\prime}$ and the singular points of the quadrics $\left\{W(\omega+j \tau) \mid \omega \in E_{2}, 0 \leqslant j \leqslant s-2\right\}$. In particular, the non-Azumaya locus is of codimension $>1$ in Spec $\mathscr{Z}$.

Proof. (a) By (4.4a) $M(p)$ contains a copy of the point module $M\left(p^{\circ}\right)$ for $k\left[u_{1}, u_{2}, u_{3}, u_{4}\right]$ where $p^{\circ}=p^{\prime}-(s-1) \tau^{\prime}$. Hence the support of $M(p)$ in $\mathbb{P}^{\prime}$ is $p^{\circ}$. Since the inclusion $E^{\prime} \rightarrow \mathbb{P}^{\prime}$ is the composition $E^{\prime} \rightarrow \operatorname{Spec} \mathscr{Z} \rightarrow \mathbb{P}^{\prime}$ and $M(p)$ is supported at a single point of $\operatorname{Spec} \mathscr{Z}$, the result follows.

(b) Let $y \in \operatorname{Spec} \mathscr{Z}$ denote the support of $F(\omega+j \tau)$. Suppose that $p, q \in E$ are such that $p+q=\omega+j \tau$ and $p-q \notin 2 \mathbb{Z} \tau$. Since there is a nonzero $A$-module map $M(p, q) \rightarrow F(\omega+j \tau), \Omega(\omega+j \tau)$ annihilates $F(\omega+j \tau)$. Hence, $y \in \mathscr{V}(\Omega(\omega+j \tau))=$ $W(\omega+j \tau)$. Since $\pi$ maps $W(\omega+j \tau)$ isomorphically onto $Q\left(\omega^{\prime}+j \tau^{\prime}\right)$ it is enough to show that $\pi(y)$ is a singular point of $Q\left(\omega^{\prime}+j \tau^{\prime}\right)$. Notice that $2\left(\omega^{\prime}+j \tau^{\prime}\right)=0$, so this quadric is singular.

Since the map $\pi$ :Spec $\mathscr{Z} \rightarrow \mathbb{P}^{\prime}$ arises from the inclusion $k\left[u_{1}, \ldots, u_{4}\right]^{(n)} \subset$ $Z\left(A^{(b)}\right), \pi(y)$ is the support of $F(\omega+j \tau)$ as a $k\left[u_{1}, u_{2}, u_{3}, u_{4}\right]^{(n)}$-module. By (4.4b) the $k\left[u_{1}, \ldots, u_{4}\right]$-module generated by $M(p, q)_{0}$ is isomorphic to the line module $M\left(p^{\circ}, q^{\circ}\right)$. Hence there is a nonzero $k\left[u_{1}, \ldots, u_{4}\right]$-module homomorphism $M\left(p^{\circ}, q^{\circ}\right) \rightarrow F(\omega+j \tau)$, so $\pi(y)$ is contained in the support of $M\left(p^{\circ}, q^{\circ}\right)$ as a $k\left[u_{1}, u_{2}, u_{3}, u_{4}\right]^{(n)}$-module. But $k\left[u_{1}, \ldots, u_{4}\right]$ is a twist of the polynomial ring $B\left(\mathbb{P}^{\prime}, 1, \mathcal{O}_{\mathbb{P}},(1)\right)$ and $M\left(p^{\circ}, q^{\circ}\right)$ is the twist of the line module for $B\left(\mathbb{P}^{\prime}, 1, \mathcal{O}_{\mathrm{P}}(1)\right)$ corresponding to the secant line $\overline{p^{\circ} q^{\circ}}$. It follows that the support of $M\left(p^{\circ}, q^{\circ}\right)$ is $\overline{p^{\circ} q^{\circ}}$ which lies on $Q\left(p^{\circ}+q^{\circ}\right)=Q\left(\omega^{\prime}+j \tau^{\prime}\right)$. As we vary $p$ and $q$, we see that $\pi(y)$ lies on infinitely many of the lines on $Q\left(\omega^{\prime}+j \tau^{\prime}\right)$, so $\pi(y)$ must be the singular point of this quadric.

(c) This follows from the discussion prior to the lemma.

THEOREM 5.12. The singular locus of Spec $\mathscr{Z}$ is the union of $E^{\prime}$ and the vertices of the singular quadrics $W(\omega+i \tau)$ for $\omega \in E_{2}$ and $0 \leqslant i \leqslant s-2$.

Proof. Let $y \in(\operatorname{Spec} \mathscr{Z}) \backslash E^{\prime} \cong\left(\mathbb{P}^{\prime} \backslash E^{\prime}\right) \times_{S^{\prime}} S$. A criterion for the singularity of a point in a fiber product is given in [7, Corollaire 17.13.6]. Since $S^{\prime}$ is a curve this criterion says that $y$ is singular if and only if the differentials $\mathrm{d} \psi$ and $\mathrm{d} \theta^{\prime}$ vanish at $\theta(y)$ and $\pi(y)$ respectively. This is equivalent to $\theta(y)$ being ramified over $S^{\prime}$ and $\pi(y)$ being singular on its fiber over $S^{\prime}$, which is the quadric $Q\left(p^{\prime}\right)$ containing it. By (5.8c), and the fact that $\pi$ is an isomorphism on each quadric, this is equivalent to $y$ being the singular point of $W(\omega+i \tau)$ for some $\omega \in E_{2}$ and $0 \leqslant i \leqslant s-2$.

To show that Spec $\mathscr{Z}$ is singular along $E^{\prime}$ we use a method suggested by $\mathrm{M}$. Artin. It suffices to show that the generic point of $E^{\prime}$ is singular. But the generic point of $E^{\prime}$ is of codimension 2, so if its local ring were regular, then $\mathscr{A}$, which is a maximal order, would be Azumaya there because the non-Azumaya locus has codimension $>1$. But the set of points where $\mathscr{A}$ is Azumaya is open, so there would be some closed points of $E^{\prime}$ where $\mathscr{A}$ is Azumaya, thus contradicting (5.11). 
Remark. The fact that the singular locus and the non-Azumaya locus are the same holds in rather general circumstances. In [9] L. LeBruyn proves that if $A$ is a positively graded, Auslander-regular algebra with the Cohen-Macaulay property (as our $A$ is), and $A$ is a finite module over a Nötherian central subalgebra, and the non-Azumaya locus of the corresponding $\mathscr{A}$ is of codimension $>1$, then the non-Azumaya locus coincides with the singular locus of $\operatorname{Spec} \mathscr{Z}$.

PROPOSITION 5.13. Spec $\mathscr{Z}$ is a rational variety, but is not isomorphic to $\mathbb{P}^{3}$.

Proof. We define a map $\varphi$ : Spec $\mathscr{Z} \rightarrow \mathbb{P}^{1} \times \mathbb{P}^{2}$ on a dense open subset of Spec $\mathscr{Z}$ as follows. The first component of $\varphi$ is the map $\theta: \operatorname{Spec} \mathscr{Z} \rightarrow S$, and the second component is the composition $\gamma \pi$, where $\gamma$ is the projection $\mathbb{P}^{\prime} \rightarrow \mathbb{P}^{2}$ with center some point $p^{\prime} \in E^{\prime}$. Since $p^{\prime}$ is in $Q\left(q^{\prime}\right)$ for each $q^{\prime} \in E^{\prime}, \gamma$ gives a birational isomorphism $Q\left(q^{\prime}\right) \rightarrow \mathbb{P}^{2}$. Hence for each $q \in E, \gamma \pi$ gives a birational isomorphism $W(q) \rightarrow \mathbb{P}^{2}$. It follows that $\varphi$ is injective, and hence an isomorphism on its domain of definition.

Since Spec $\mathscr{Z}$ is singular, it is certainly not isomorphic to $\mathbb{P}^{3}$.

\section{Acknowledgements}

S.P.S. thanks the University of Auckland for its hospitality while part of this work was carried out, and is grateful for financial support under NSF grant DMS9100316.

\section{References}

1. Artin, M.: Geometry of quantum planes, in D. Haile and J. Osterburg (eds), Azumaya Algebras, Actions and Modules Contemp. Math. 124, Amer. Math. Soc., Providence, 1992. 1-15.

2. Artin, M. and Schelter, W.: Graded algebras of global dimension 3, Adv. Math. 66 (1987), 171-216.

3. Artin, M., Schelter, W., and Tate, J.: The centers of 3-dimensional Sklyanin algebras, Proc. Barsotti Memorial Conference, 1991, Preprint, 1992.

4. Artin, M., Tate, J., and Van den Bergh, M.: Some algebras associated to automorphisms of elliptic curves, The Grothendieck Festschrift, Vol. 1, Birkhäuser, Basel, 1990, pp. 33--85.

5. Artin, M., Tate, J., and Van den Bergh, M.: Modules over regular algebras of dimension 3, Invent. Math. 106 (1991), 335-388.

6. Artin, M. and Van den Bergh, M.: Twisted homogeneous coordinate rings, J. Algebra 133 (1990), 249-271.

7. Grothendieck, A. and Dieudonné, J.: Eléments de géometrie algébrique, Publ. Math. Inst. des Hautes Études Sci., 1960-67.

8. Hartshorne, R.: Algebraic Geometry, Springer-Verlag, New York, 1977.

9. Lebruyn, L.: Central singularities of certain quantum spaces, Preprint, Univ. of Antwerp, 1993.

10. Levasseur, T. and Smith, S. P.: Modules over the 4-dimensional Sklyanin algebra, Bull. Soc. Math. France 121 (1993), 35-90.

11. Mumford, D.: Abelian Varieties, Tata Studies in Math., Oxford Univ. Press, 1970.

12. Odesskii, A. V. and Feigin, B. L.: Elliptic Sklyanin algebras, Funktsional Anal. i Prilozhen 23 (1989), 45-54.

13. Smith, S. P. and Stafford, J. T.: Regularity of the four dimensional Sklyanin algebra, Compositio Math. 83 (1992), 259-289. 
14. Smith, S. P.: The 4-dimensional Sklyanin algebra at points of finite order, Preprint, University of Washington, 1992.

15. Stafford, J. T.: Auslander-regular algebras and Maximal Orders, J. Lond. Math. Soc., to appear.

16. Tate, J. and Van den Bergh, M.: Homological properties of Sklyanin algebras, Preprint, 1993.

17. Zhang, J.: Twisted graded algebras and equivalences of graded categories, Proc. Lond. Math. Soc., to appear. 